\title{
Fusion nuclear science facilities and pilot plants based on the spherical tokamak
}

\author{
J. E. Menard \\ jmenard@pppl.gov \\ T. Brown \\ L. El-Guebaly \\ M. Boyer \\ J. Canik
}

See next page for additional authors

Follow this and additional works at: https://scholarworks.wm.edu/aspubs

\section{Recommended Citation}

Menard, J. E.; Brown, T.; El-Guebaly, L.; Boyer, M.; Canik, J.; Colling, B.; Raman, R.; Wang, Z.; Zhai, Y.; Buxton, P.; Covele, B.; D'Angelo, C.; Davis, A.; Gerhardt, S.; Gryaznevich, M.; Harb, M.; Hender, T. C.; Kaye, S.;

Kingham, D.; Kotschenreuther, M.; Mahajan, S.; Maingi, R.; Marriott, E.; Meier, E. T.; and Mynsberge, L., Fusion nuclear science facilities and pilot plants based on the spherical tokamak (2016).

10.1088/0029-5515/56/10/106023

This Article is brought to you for free and open access by the Arts and Sciences at W\&M ScholarWorks. It has been accepted for inclusion in Arts \& Sciences Articles by an authorized administrator of W\&M ScholarWorks. For more information, please contact scholarworks@wm.edu. 


\section{Authors}

J. E. Menard, T. Brown, L. El-Guebaly, M. Boyer, J. Canik, B. Colling, R. Raman, Z. Wang, Y. Zhai, P. Buxton, B. Covele, C. D'Angelo, A. Davis, S. Gerhardt, M. Gryaznevich, M. Harb, T. C. Hender, S. Kaye, D. Kingham, M. Kotschenreuther, S. Mahajan, R. Maingi, E. Marriott, E. T. Meier, and L. Mynsberge 
PAPER

\section{Fusion nuclear science facilities and pilot plants based on the spherical tokamak}

To cite this article: J.E. Menard et al2016 Nucl. Fusion 56106023

View the article online for updates and enhancements.

\section{Related content}

- Overview of the physics and enqineering design of NSTX upgrade

J.E. Menard, S. Gerhardt, M. Bell et al.

Prospects for pilot plants based on the tokamak, spherical tokamak and stellarator J.E. Menard, L. Bromberg, T. Brown et al.

- ITER conceptual design ${ }^{*}$

K. Tomabechi, J.R. Gilleland, Yu.A. Sokolov et al.

\section{Recent citations}
DIII-D Research to Prepare for Steady State Advanced Tokamak Power Plants R. J. Buttery et al
- Structure and Properties of High-Hardness Silicide Coatings on Cemented Carbides for High Temperature Applications Samuel Humphry-Baker and Jessica Marshall
Nuclear Problems of Thermonuclear Power Generation
V. V. Orlov and L. I. Ponomarev 


\title{
Fusion nuclear science facilities and pilot plants based on the spherical tokamak
}

\author{
J.E. Menard ${ }^{1}$, T. Brown ${ }^{1}$, L. El-Guebaly ${ }^{2}$, M. Boyer ${ }^{1}$, J. Canik ${ }^{3}$, B. Colling ${ }^{4}$, \\ R. Raman ${ }^{5}$, Z. Wang ${ }^{1}$, Y. Zhai ${ }^{1}$, P. Buxton ${ }^{6}$, B. Covele ${ }^{7}$, C. D'Angelo ${ }^{2}$, \\ A. Davis ${ }^{2}$, S. Gerhardt ${ }^{1}$, M. Gryaznevich ${ }^{6}$, M. Harb' ${ }^{2}$, T.C. Hender ${ }^{4}$, S. Kaye ${ }^{1}$, \\ D. Kingham ${ }^{6}$, M. Kotschenreuther ${ }^{7}$, S. Mahajan ${ }^{7}$, R. Maingi ${ }^{1}$, E. Marriott ${ }^{2}$, \\ E.T. Meier ${ }^{8,10}$, L. Mynsberge ${ }^{2}$, C. Neumeyer ${ }^{1}$, M. Ono ${ }^{1}$, J.-K. Park ${ }^{1}$, \\ S.A. Sabbagh ${ }^{9}$, V. Soukhanovskii ${ }^{10}$, P. Valanju ${ }^{7}$ and R. Woolley ${ }^{1}$ \\ 1 Princeton Plasma Physics Laboratory, Princeton, NJ, USA \\ 2 University of Wisconsin, Madison, WI, USA \\ 3 Oak Ridge National Laboratory, Oak Ridge, TN, USA \\ ${ }^{4}$ CCFE, Culham Science Centre, Abingdon, Oxfordshire, UK \\ ${ }^{5}$ University of Washington, Seattle, WA, USA \\ 6 Tokamak Energy Ltd, Milton Park, Oxfordshire, UK \\ ${ }^{7}$ University of Texas at Austin, Austin, TX, USA \\ 8 College of William and Mary, Williamsburg, VA, USA \\ 9 Columbia University, New York, NY, USA \\ ${ }^{10}$ Lawrence Livermore National Laboratory, Livermore, CA, USA \\ E-mail: jmenard@pppl.gov
}

Received 29 March 2016, revised 29 June 2016

Accepted for publication 12 July 2016

Published 16 August 2016

\begin{abstract}
A fusion nuclear science facility (FNSF) could play an important role in the development of fusion energy by providing the nuclear environment needed to develop fusion materials and components. The spherical torus/tokamak (ST) is a leading candidate for an FNSF due to its potentially high neutron wall loading and modular configuration. A key consideration for the choice of FNSF configuration is the range of achievable missions as a function of device size. Possible missions include: providing high neutron wall loading and fluence, demonstrating tritium self-sufficiency, and demonstrating electrical self-sufficiency. All of these missions must also be compatible with a viable divertor, first-wall, and blanket solution. ST-FNSF configurations have been developed simultaneously incorporating for the first time: (1) a blanket system capable of tritium breeding ratio TBR $\approx 1$, (2) a poloidal field coil set supporting high elongation and triangularity for a range of internal inductance and normalized beta values consistent with NSTX/NSTX-U previous/planned operation, (3) a long-legged divertor analogous to the MAST-U divertor which substantially reduces projected peak divertor heat-flux and has all outboard poloidal field coils outside the vacuum chamber and superconducting to reduce power consumption, and (4) a vertical maintenance scheme in which blanket structures and the centerstack can be removed independently. Progress in these ST-FNSF missions versus configuration studies including dependence on plasma major radius $R_{0}$ for a range $1 \mathrm{~m}-2.2 \mathrm{~m}$ are described. In particular, it is found the threshold major radius for $\mathrm{TBR}=1$ is $R_{0} \geqslant 1.7 \mathrm{~m}$, and a smaller $R_{0}=1 \mathrm{~m} \mathrm{ST}$ device has TBR $\approx 0.9$ which is below unity but substantially reduces $\mathrm{T}$ consumption relative to not breeding. Calculations of neutral beam heating and current drive for non-inductive ramp-up and sustainment are described. An $A=2, R_{0}=3 \mathrm{~m}$ device incorporating high-temperature superconductor toroidal field coil magnets capable of high neutron fluence and both tritium and electrical self-sufficiency is also presented following systematic aspect ratio studies.
\end{abstract}


Keywords: fusion nuclear science facility, pilot plant, spherical tokamak, tritium breeding, negative neutral beams, super-X divertor, high-temperature superconductors

(Some figures may appear in colour only in the online journal)

\section{Introduction}

There are several possible pathways from successful demonstration of a burning plasma in ITER to a commercial power plant. These different pathways are influenced by the number of missions to be carried forward in the device(s) [1] following ITER. Such missions include: providing high neutron wall loading and fluence, demonstrating tritium selfsufficiency, and demonstrating electrical self-sufficiency. Which missions are chosen has a strong influence on device configuration and size. One option is a fusion demonstration power plant (DEMO) [2] with an engineering gain (i.e. ratio of electrical power produced to electrical power consumed) $Q_{\text {eng }} \sim 3-5$ and other parameters approaching those of a firstof-a-kind power plant. Another option is a 'pilot plant' which is a potentially attractive next-step towards fusion commercialization by demonstrating generation of a small amount of net electricity $Q_{\text {eng }} \geqslant 1$ as quickly as possible and in as small a facility as possible in a configuration directly scalable to a power plant [3]. However, to advance directly from ITER to a DEMO or pilot plant there are significant challenges to achieving net electricity and tritium fuel production-in particular the blanket technology used for thermal power conversion and tritium breeding. Such challenges have motivated consideration of a fusion nuclear science facility (FNSF)/ component test facility (CTF) [4-10] to provide a facility to aid in the development of fusion energy by providing the nuclear environment needed to develop fusion materials and components but without the risk, size, and cost associated with the goal of $Q_{\mathrm{eng}} \geqslant 1$. The goal of such a device would be to provide fusion-relevant neutron wall loading $W_{\mathrm{n}} \geqslant 1 \mathrm{MW} \mathrm{m}^{-2}$, neutron fluence $\geqslant 6 \mathrm{MW} \mathrm{yr} \mathrm{m}^{-2}$, component testing area of 5-10 $\mathrm{m}^{2}$, and continuous on-time (i.e. steady-state operation) for durations in the range of $10^{6} \mathrm{~s}$ [11].

The spherical torus/tokamak (ST) is a leading candidate for the FNSF/CTF application due to its potentially high neutron wall loading and modular configuration. However, several key questions have not previously been addressed for an ST-based FNSF, including:

1. How large must an ST device be to achieve tritium breeding ratio $\mathrm{TBR} \geqslant 1$ ?

2. What is the impact of the divertor configuration and blanket penetrations on TBR?

3. How much externally supplied tritium would be needed if $\mathrm{TBR} \leqslant 1$ ?

4. What are the device and component lifetimes?

5. How do high-temperature superconductors (HTS) influence FNSF options?

The methodology used to address these questions is iterative and begins with zero-dimensional systems studies to estimate fusion performance levels as a function of device size and physics assumptions for stability, confinement, and heating and current drive efficiency. This is followed by free-boundary equilibrium calculations to identify poloidal field (PF) coil locations and currents, then detailed CAD-based device layouts, and then detailed 3D neutronics calculations of neutron wall loading, shielding, and tritium breeding. The results of the neutronics calculations then influence the design assumptions and device layout for the next iteration.

Based on such techniques, recent studies have for the first time identified ST-FNSF configurations simultaneously incorporating: (1) tritium self-sufficiency, i.e. a blanket system capable of tritium breeding ratio TBR $\approx 1$, (2) a poloidal field (PF) coil set supporting high elongation $\kappa$ and triangularity $\delta$ for a range of internal inductance $l_{\mathrm{i}}$ and normalized beta $\beta_{\mathrm{N}}$ values consistent with NSTX/NSTX-U previous/planned operation, (3) a long-legged/super-X divertor analogous to the MAST-U divertor [12] which substantially reduces projected peak divertor heat-flux and has all outboard equilibrium PF coils outside the vacuum chamber and as superconducting to reduce power consumption, and (4) a vertical maintenance scheme in which blanket structures and the centerstack (CS) can be removed independently. TRANSP/NUBEAM [13-15] calculations of neutral beam heating and current drive in support of full non-inductive operation (see section 2.1.5) are also incorporated including the layout of the neutral beams and associated penetrations in the blankets. A key finding for copper-TF-based ST-FNSF devices is that the threshold major radius for TBR $\approx 1$ is $R_{0}=1.7 \mathrm{~m}$, and a smaller $R_{0}=1 \mathrm{~m} \mathrm{ST}$ device has $\mathrm{TBR} \approx 0.9$ which is below unity but substantially reduces $T$ consumption relative to not breeding. Further, leveraging the finding of high TBR at low-A using only/mostly outboard breeding, very high current density HTS toroidal field coils are found to offer the possibility of low-A $(A=1.8-2.2)$ FNSF devices that achieve the pilot plant mission of electrical self-sufficiency and have reduced TF magnet mass albeit with the inclusion of inboard shielding and larger major radius.

The organization of the remainder of this paper is as follows: section 2 describes the overall physics design assumptions for ST-FNSF using $\mathrm{Cu}$ TF magnets, section 3 describes ST-FNSF device layout and maintenance issues, section 4 describes neutronics modelling including shielding and tritium breeding ratio calculations, section 5 discusses low-A pilot plant concepts using HTS toroidal field coils to reduce magnet resistive power losses, and section 6 summarizes the results including answers to the above key questions posed for ST-based FNSFs and pilot plants.

\section{Physics design}

The physics design of the ST-based FNSF described here is carried out in multiple steps. First, approximate zero-dimension/ 
global models of ST-FNSF performance are utilized to define operating scenarios and equilibrium requirements. Second, the results from the zero-dimensional (0D) models are used to constrain free-boundary equilibrium calculations, then such calculations are used to identify poloidal field (PF) coil locations, currents, and current densities along with plasma facing component (PFC) and limiter locations consistent with approximate models for shielding, blankets, ports, and other device components. Lastly, the PF coil locations and other parameters are used in free-boundary TRANSP/NUBEAM calculations of plasma kinetic profiles consistent with fullnon-inductive operation utilizing bootstrap (BS) current and neutral beam injection (NBI) current drive (CD). The NBI-CD efficiency and profile dependence on NBI tangency radius is then used to guide NBI port layout which in turn impacts the overall device layout and tritium breeding capabilities as described in section 4.2. The physics design results from the OD models, free boundary equilibrium calculations, and TRANSP/NUBEAM simulations are described in the subsections that follow.

\subsection{Zero-dimensional systems studies}

In this section, zero-dimensional/global scalings are used to guide the first iteration of the physics design of an ST-based FNSF. These scaling calculations have been benchmarked against TRANSP simulations as described in section 2.4. The choice of aspect ratio and toroidal field is explained, the impact of vertical and kink instabilities are discussed, and the energy confinement assumptions, heating and current drive tools, and choice of operating density are described.

2.1.1. Choice of aspect ratio and toroidal field. The deuterium-tritium (D-T) fusion power $P_{\mathrm{f}}$ in a magnetic confinement system scales as $P_{\mathrm{f}} \propto n^{2}\langle\sigma v\rangle_{\mathrm{DT}}$ [16] which for ion temperatures in the range of $8-30 \mathrm{keV}$ is approximately proportional to $p^{2}=(n T)^{2} \propto \beta_{\mathrm{T}}^{2} B_{\mathrm{T} 0}^{4}$ where $\beta_{\mathrm{T}} \equiv 2 \mu_{0}\langle p\rangle / B_{\mathrm{T} 0}^{2}$, $\langle p\rangle$ is the volume-averaged plasma pressure, and $B_{\mathrm{T} 0}$ is the vacuum toroidal field at the plasma geometric center. For steady-state tokamaks/STs it is anticipated that a majority of the plasma current must be provided by the bootstrap current, and it can be shown that [17] $\beta_{\mathrm{T}} \sim A^{-1 / 2}\left(1+\kappa^{2}\right) \beta_{\mathrm{N}}^{2} / f_{\mathrm{BS}}$ where the plasma aspect ratio $A \equiv \epsilon^{-1} \equiv R_{0} / a, R_{0}$ is the radius of the plasma geometric center, $a$ is the plasma minor radius, $\kappa$ is the plasma boundary elongation, $f_{\mathrm{BS}}$ is the bootstrap current fraction, and the normalized beta $\beta_{\mathrm{N}} \equiv \beta_{\mathrm{T}} a B_{\mathrm{T}} / I_{\mathrm{P}}\left(\% \mathrm{mT} \mathrm{MA}^{-1}\right)$ where $I_{\mathrm{P}}$ is the plasma current. From this scaling, and since typically $\kappa^{2} \gg 1$, it is evident that $P_{\mathrm{f}}$ scales approximately as $P_{\mathrm{f}} \propto \epsilon\left(\kappa \beta_{\mathrm{N}} B_{\mathrm{T}}\right)^{4}$. This scaling implies achieving high $\kappa, \beta_{\mathrm{N}}$, and $B_{\mathrm{T}}$ are arguably of equal importance for achieving high fusion power density at fixed bootstrap fraction. The maximum achievable $\kappa$ and $\beta_{\mathrm{N}}$ are known to increase as the aspect ratio is decreased [17-20], whereas the maximum $B_{\mathrm{T}}$ in the plasma decreases as the aspect ratio is reduced due to a combination of field/stress and/or current density limits at the central toroidal field magnet, $1 / \mathrm{R}$ variation of the toroidal field, and the required thickness of inboard shielding. For proposed
NSTX experimental $\kappa$ vs. $I_{i}$ operating space

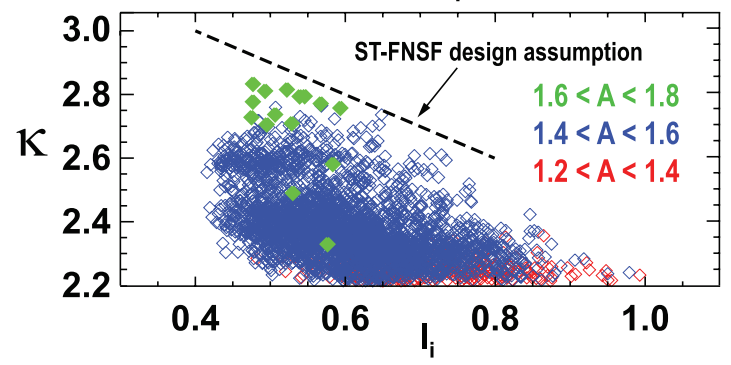

Figure 1. Achieved NSTX elongation $\kappa$ versus internal inductance $l_{\mathrm{i}}$ and design assumption for ST-FNSF (dashed line)

ST-FNSF devices with normally conducting (copper) toroidal field coils and relatively thin inboard/high-field-side shielding $(\leqslant 20 \mathrm{~cm})$ between the plasma facing components and the TF magnet, optimizations based on minimizing the center-post mass or electrical power consumption or cost of electricity $[5,18,21]$ find the range of optimal aspect ratio $A=1.5-2$. For the studies in this paper, a narrower range of aspect ratios in the center of this range is chosen, namely $A=1.7-1.8$, and the toroidal field is chosen to be $B_{\mathrm{T}}=3 \mathrm{~T}$ similar to configurations studied most recently by Peng and co-workers [22] using a water-cooled center-post capable of providing $B_{\mathrm{T}} \geqslant 3 \mathrm{~T}$ including nuclear heating from neutron wall loads $\leqslant 2 \mathrm{MW} \mathrm{m}^{-2}$ $[23,24]$. The choice of a constant $B_{\mathrm{T}}=3 \mathrm{~T}$ ensures $q^{*}$ is near/above 3 for the range of configurations studied (see section 2.1.3 for more detail) while also staying within magnet current density limits and minimizing TF resistive power dissipation.

2.1.2. Vertical stability limits. The maximum stable $\kappa$ is primarily a function of the plasma internal inductance $l_{\mathrm{i}}$, conducting wall position, and aspect ratio, and lower aspect ratio has a higher natural elongation $[17,25]$. For steady-state tokamaks with a majority of the plasma current provided by the bootstrap current, the $l_{\mathrm{i}}$ is more strongly influenced by the plasma pressure profile and broad pressure profiles generate bootstrap current at larger minor radius which reduces $l_{\mathrm{i}}$. Figure 1 shows the $\mathrm{x}$-point elongation achieved in NSTX as a function of $l_{\mathrm{i}}$ for several groupings of aspect ratio. The dashed line is a linear fit to $\kappa$ values slightly above the upper-bound of the NSTX data and is given by $\kappa_{x-\mathrm{ST}}=3.4-l_{\mathrm{i}}$. This formula is used to constrain the ST-FNSF equilibrium $\kappa$ for a range $l_{\mathrm{i}}$ values $=0.4-0.8$. It is anticipated that improved vertical position identification and control [26] will enable increases in stable elongation of $5-10 \%$ in NSTX-U and ST-FNSF. Using NSTX data and TRANSP modeling of NSTX-U as a guide $[27,28]$, the most probable thermal pressure peaking factor $p(0) /\langle p\rangle=1.7-2$ in H-mode plasmas corresponding to $l_{\mathrm{i}}=0.5-0.65$ and $\kappa \leqslant 2.9-2.75$ using the formula above. Assuming the corresponding total pressure peaking factor does not exceed 2.5 , this range of thermal $p(0) /\langle p\rangle$ and $l_{\mathrm{i}}$ minimized disruptivity in NSTX as shown in figure 2 and is used to guide the chosen poloidal field coil set for the ST-FNSF configurations studied here.

A potentially important difference between NSTX/ NSTX-U and ST-FNSF achievable elongation is the close 

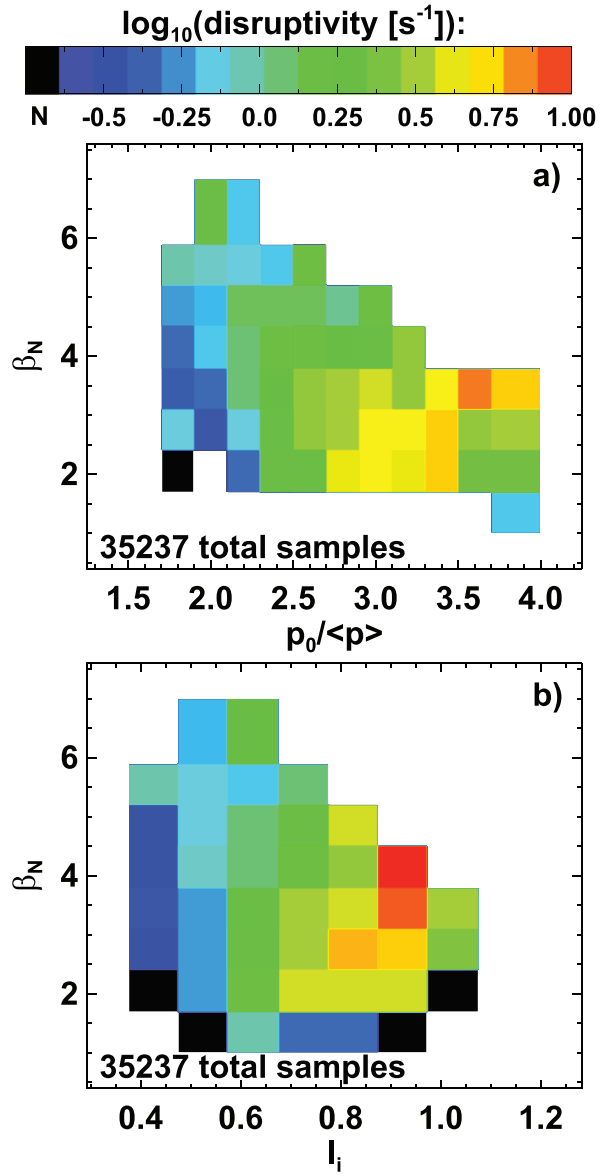

Figure 2. NSTX disruptivity versus $\beta_{\mathrm{N}}$ and (a) total pressure profile peaking and $(b)$ internal inductance $l_{\mathrm{i}}$ (figure reproduced with permission [43]).

proximity of the passive stabilizing conductors in NSTX-U [27] relative to what may be possible in ST-FNSF due to limits on disruption-induced electromagnetic forces to the activelycooled blanket first-wall [29-31]. A possible disadvantage for small minor radius $(a \leqslant 1 \mathrm{~m})$ devices with $\approx 1 \mathrm{~m}$ thick breeding blankets is that any toroidally electrically conducting passive structure may need to be located at least $0.35-0.5 \mathrm{~m}$ behind the first wall for acceptable tritium breeding, and thus the wall is comparatively farther away than for a larger device, i.e. $r_{\text {wall }} / a$ is potentially larger for a smaller device. To obtain a preliminary assessment of such effects for ST-FNSF, the timedependent electromagnetic and equilibrium reconstruction code LRDFIT [32-34] has been utilized to calculate vertical instability growth rates and dynamics using a rigid plasma model validated against NSTX open-loop vertical growth rate data. Plasma deformation effects $[35,36]$ are potentially important for closed-loop feedback control and will be investigated in future work.

One of the most stringent requirements for the vertical control system is the vertical position recoverability of a plasma that is allowed to drift or is vertically shifted rapidly by an internal plasma/MHD event. Controlled recovery of plasmas vertically shifted by $\Delta Z \geqslant 5 \%$ of the minor radius is considered acceptably robust control [37]. ITER will use a dedicated in-vessel control coil power supply to mitigate such vertical
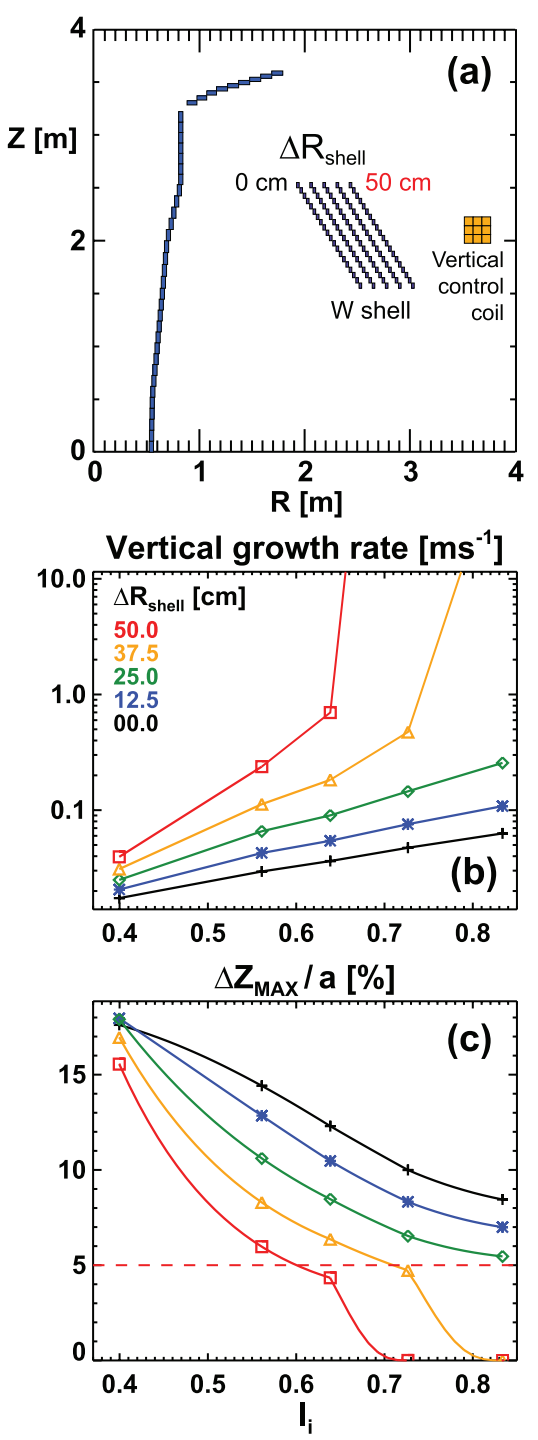

Figure 3. ST-FNSF vertical stability scan showing $(a)$ outboard tungsten shell radial positions $\Delta R_{\text {shell }}$ relative to the nominal first-wall position, other passive stabilizer positions, and vertical control coil location, $(b)$ vertical instability open-loop growth rate versus shell position and internal inductance, and $(c)$ maximum recoverable vertical position shift.

position transients, and this supply will have a very rapid voltage rise time of $1 \mathrm{~ms}$ with a peak inductive power capacity of $2.4 \mathrm{kV} \times 60 \mathrm{kA}=144$ MVA [38]. Since the plasma current of the larger ST-FNSF devices considered here is as high as $15 \mathrm{MA}$ and therefore comparable to ITER plasma current levels, it is assumed a similar power supply could be utilized for ST-FNSF vertical transient suppression.

A potentially effective means of providing vertical instability suppression while reducing first-wall disruption loads is to have a two-layer blanket structure with radial space between layers for passive stabilizers to be incorporated $[39,40]$. Tungsten shells $2-3 \mathrm{~cm}$ thick can provide sufficient wall conductivity even at elevated temperatures $\left(600{ }^{\circ} \mathrm{C}\right.$ is assumed here) and with acceptable reductions in TBR provided the shell is sufficiently far behind the first-wall as shown in figure 38. Figure 3 shows the results of vertical stability analysis of $R_{0}=1.7 \mathrm{~m} \mathrm{ST}-\mathrm{FNSF}$ equilibria with $I_{\mathrm{P}}=11.6 \mathrm{MA}$. 
The 5 equilibria treated $\operatorname{span} l_{\mathrm{i}}=0.4-0.85$ and are a subset of the $\beta_{\mathrm{N}}=5$ equilbria from figures 15 and 16 . Figure 3(a) shows the up-down symmetric passive conductors and (antiseries) vertical position control coil used for this analysis. The toroidally electrically continuous conductors include the inboard vacuum vessel, the top and bottom divertor/breeding regions, and the outboard $W$ shell. As shown in figure 3(b), the vertical mode growth rates are sub-Alfvenic for $\Delta R_{\text {wall }}=0$ $-50 \mathrm{~cm}$ for $l_{\mathrm{i}} \leqslant 0.65$. Figure $3(c)$ shows the $\Delta Z_{\max } / a$ that can be recovered with a step voltage request consistent with an ITER-like 144 MVA power supply capability. As seen in this figure, acceptable vertical transient control $\left(\Delta Z_{\max } \geqslant 5 \%\right)$ is achievable for all $l_{\mathrm{i}}$ if $\Delta R_{\text {shell }} \leqslant 25 \mathrm{~cm}$. However, the higher $l_{\mathrm{i}} \geqslant 0.7$ cases become progressively ideal-wall unstable for $\Delta R_{\text {shell }} \geqslant 37.5 \mathrm{~cm}$. Thus, an ST-FNSF operating $l_{\mathrm{i}} \leqslant 0.6$ should have acceptable stable vertical transient control with $\Delta R_{\text {shell }}=$ $50 \mathrm{~cm}$ desired for maximizing tritium breeding and assumed for neutronics analysis, but higher $l_{\mathrm{i}}$ operation would require either reduced elongation or an improved wall stabilization configuration.

One possible approach to improving wall stabilization is to make the outboard blanket first-wall (FW) electrically conductive to increase the wall coverage and decrease the distance between the wall and the plasma. Figure 4(a) shows the passive conductors and control coil model used for this scenario. The toroidally electrically continuous conductors include the inboard vacuum vessel, the top and bottom divertor/breeding regions, and the outboard blanket $\mathrm{FW}$ conducting structure. The outboard breeding blanket metallic structures are assumed to be constructed of the reduced activation ferritic-martensitic (RAFM) steel EUROFER 97 which has a relatively high resistivity of $1.1 \mu \Omega \mathrm{m}$ at an assumed operating temperature of $550{ }^{\circ} \mathrm{C}$ [41]. Further, the first-wall of the dual-cooled leadlithium (DCLL) [42] blankets assumed for the ST-FNSF design have a relatively thin He-cooled FW with approximately $1.3 \mathrm{~cm}$ effective radial width of steel for carrying toroidal current. In addition, the side-walls of the blanket module FW further increase the path-length and effective resistance if toroidal current flow is allowed.

Assuming the first-wall is allowed to carry toroidal current, figure $4(b)$ shows the vertical instability open-loop growth rates at 5 different blanket FW radial positions $\Delta R_{\text {wall }}$ relative to the nominal FW position. As shown in figure $4(b)$, the growth rates are $0.1-0.2 \mathrm{~ms}^{-1}$ for the lowest $l_{\mathrm{i}}$ value $=0.4$ and are weakly dependent on wall position, while at the highest $l_{\mathrm{i}}=0.84$ the plasma becomes ideally unstable at $\Delta R_{\text {wall }}=50 \mathrm{~cm}$. Thus, even with increased wall poloidal coverage, the $l_{\mathrm{i}}=0.84$ case is ideally unstable when the wall is shifted $50 \mathrm{~cm}$ outward. As seen in figure $4(c), l_{\mathrm{i}}=0.4$ is robustly stable for all wall positions treated, the nominal operating $l_{\mathrm{i}}=0.5-0.65$ is at or somewhat below acceptable for the nominal first-wall location $\Delta R_{\text {wall }}=0 \mathrm{~cm}$ (black curve), and importantly, all $l_{\mathrm{i}}$ values are at least potentially recoverable for $\Delta R_{\text {wall }}=0 \mathrm{~cm}$. However, the nominal operating $l_{\mathrm{i}}=0.5-0.65$ lies below acceptable control for $\Delta R_{\text {wall }} \leqslant 0.375 \mathrm{~m}$ (orange curve), and $l_{\mathrm{i}} \geqslant 0.7$ is not recoverable for $\Delta R_{\text {wall }}=50 \mathrm{~cm}$ (red curve).
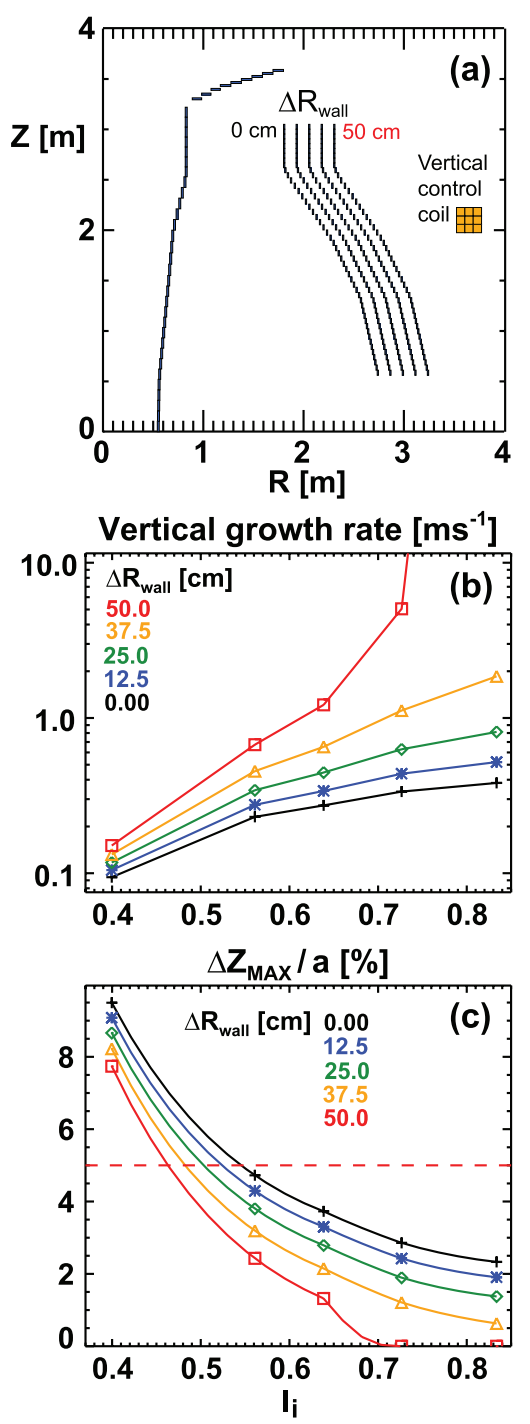

Figure 4. ST-FNSF vertical stability scan showing $(a)$ outboard first-wall radial positions $\Delta R_{\text {wall }}$ relative to the nominal first-wall position, other passive stabilizer positions, and vertical control coil location, $(b)$ vertical instability open-loop growth rate versus wall position and internal inductance, and $(c)$ maximum recoverable vertical position shift.

The results of figures 3 and 4 together indicate that robust control/recovery from vertical transients at elevated $l_{\mathrm{i}}$ requires either additional stabilization or a lower elongation than indicated by maximum achievable NSTX/NSTX-U $\kappa_{x}$ versus $l_{\mathrm{i}}$ trends shown in figure 1 . Figure 5 shows potential options for increasing vertical transient stability assuming the blanket first-wall at $\Delta R_{\text {wall }}=0 \mathrm{~cm}$ can carry toroidal current. Figure 5(a) shows the passive conductor and feedback coil locations assumed in this optimization analysis. First, figure $5(b)$ shows that the inclusion of the toroidally continuous inboard (IB) copper conductors of the single-turn toroidal field coil TF can reduce the open-loop vertical growth rate and significantly increase $\Delta Z_{\max } / a$ at low $l_{\mathrm{i}}$ as shown in figure 5(c). A substantially larger decrease in growth-rate is achieved by doubling the effective thickness of the RAFM first wall to $2.6 \mathrm{~cm}$ which doubles the conductivity of these components. As shown in the red curve in figure 5(c) for the 

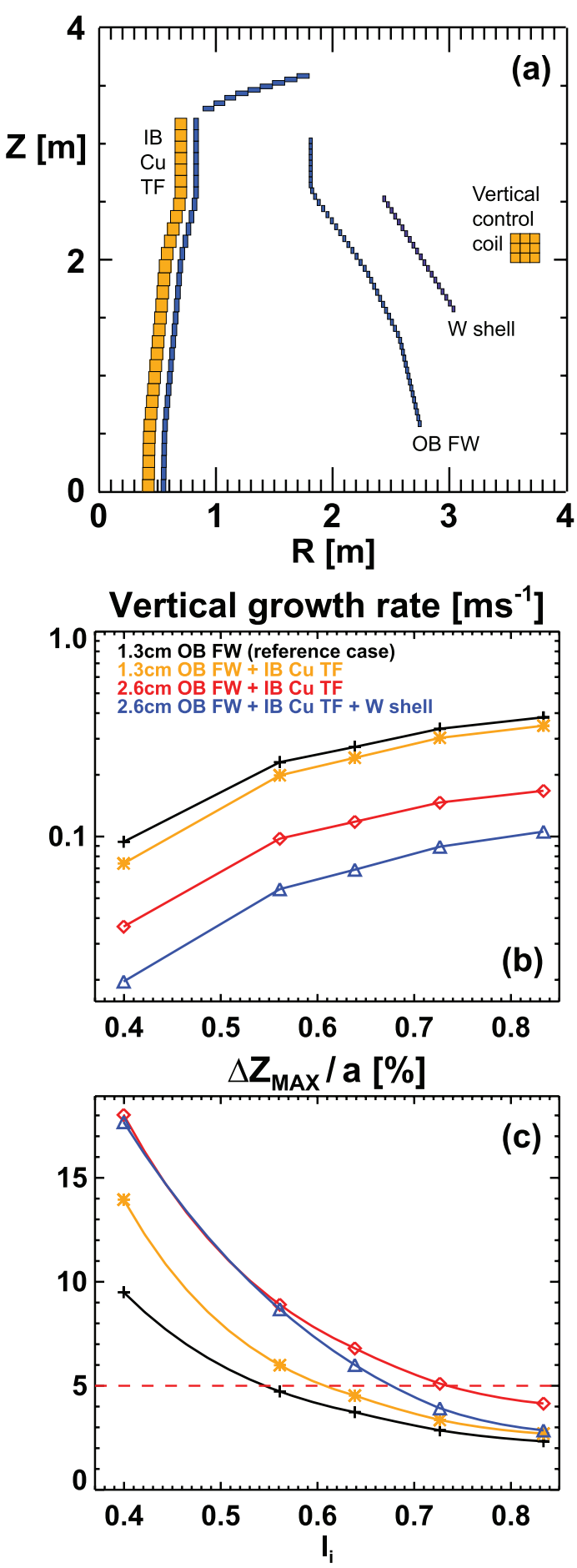

Figure 5. ST-FNSF vertical stability scan showing $(a)$ passive stabilizer and vertical control coil locations, $(b)$ vertical instability open-loop growth rate versus internal inductance and varied passive conductor assumptions, and $(c)$ maximum recoverable vertical position shift.

case with thicker $(2.6 \mathrm{~cm})$ blanket $\mathrm{FW}$ and including the IB $\mathrm{Cu}$ TF, $\Delta Z_{\max } / a \geqslant 4 \%$ for all $l_{\mathrm{i}}$ values indicating significantly improved overall vertical transient control. Placing a $2 \mathrm{~cm} W$ shell at $\Delta R_{\text {wall }}=50 \mathrm{~cm}$ further reduces the growth rates to relatively low values of $10-100 \mathrm{~s}^{-1}$ as evident in figure $5(b)$. However, the vertical transient controllability is actually degraded at higher $l_{\mathrm{i}}$ as shown in figure $5(c)$ because shielding
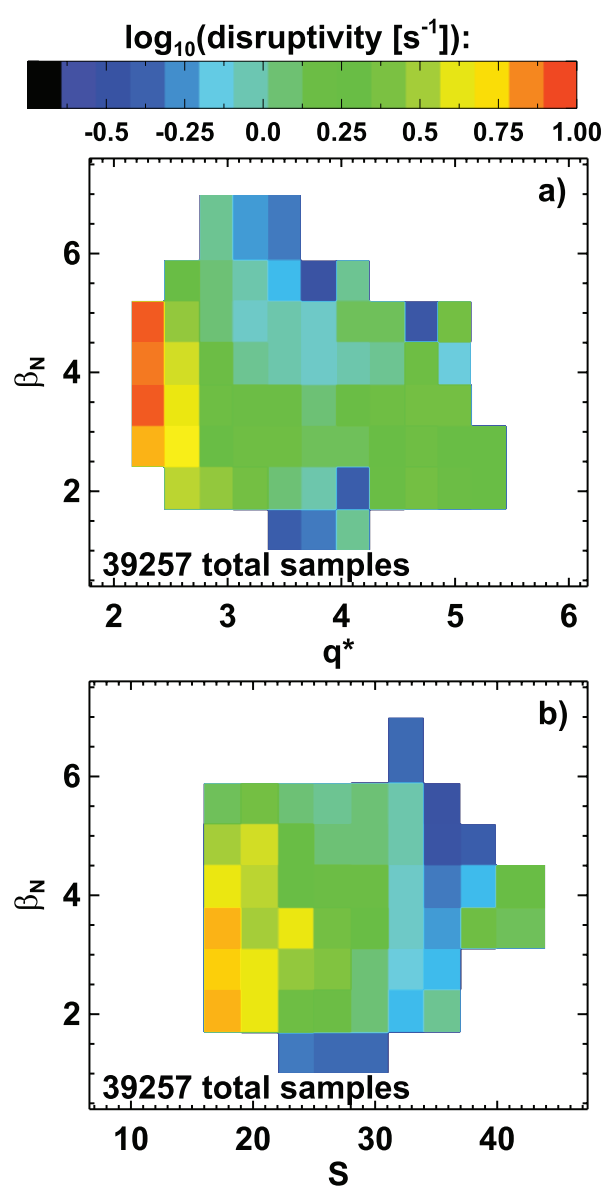

Figure 6. NSTX disruptivity versus $\beta_{\mathrm{N}}$ and $(a)$ kink safety factor $q^{*}$ and $(b)$ shaping factor $S$ (figure reproduced with permission [43]).

from the $W$ shell delays penetration of the control coil field seen by the plasma. Finally, if the increased FW thickness shown in figure 5 significantly degraded TBR, vanadium with roughly $2 \times$ the conductivity of RAFM steel is a potentially attractive FW material for vertical stabilization. Overall, either a $W$ shell at $\Delta R_{\text {wall }} \leqslant 50 \mathrm{~cm}$ or a thin toroidally conductive RAFM steel first-wall at $\Delta R_{\text {wall }}=0$ can provide marginal to acceptable vertical transient control for reference scenario $l_{\mathrm{i}}$ values $\leqslant 0.6$, while acceptable control for higher $l_{\mathrm{i}}$ values requires either $W$ shells sufficiently close to the plasma, a sufficiently conductive first-wall, and/or reduced elongation. In the design and analysis that follows it is assumed that sufficient stabilization can be provided to approach or achieve the upper-bound elongation shown in figure 1 .

2.1.3. Kink stability limits. Current-driven and pressuredriven kink modes set strong limits on the accessible fusion performance in tokamaks. The safety factor parameter $q^{*}=\epsilon\left(1+\kappa^{2}\right) \pi a B_{\mathrm{T} 0} / \mu_{0} I_{\mathrm{P}}$ is a useful metric for currentdriven kink stability [20], and analysis of NSTX disruption rates [43] shows a significant increase in disruptivity when $q^{*}<2.8$ as shown in figure 6(a). This implies any STFNSF operating point should maintain $q^{*} \geqslant 2.8$. Importantly, figure 6(a) also shows that for $q^{*}>3$ in NSTX, disruptivity does not necessarily increase at high $\beta_{\mathrm{N}}$, but instead 
moderately decreases as $\beta_{\mathrm{N}}$ is increased from 3-4 to 4-6. Figure $6(b)$ shows that NSTX plasmas with $A \leqslant 1.7$ required high shaping factor $S \equiv q_{95} I_{\mathrm{P}} / a B_{\mathrm{T}} \geqslant 20$ to reduce disruptivity, and $S \geqslant 30$ combined with $\beta_{\mathrm{N}} \geqslant 4.5$ is most favorable for low disruptivity. Such $\beta_{\mathrm{N}}$ values are near or moderately above the typical $n=1$ no-wall stability limits for NSTX plasmas [17, 20, 44-47] and for projected limits for NSTXU plasmas [28, 48]. RWM stabilization in ST-FNSF (if necessary) is assumed to be provided by either passive/kinetic stabilization [46, 49-59] and/or active RWM feedback control [60-63]. Combined/dual-purpose low- $n$ error-field correction and RWM feedback control coils in ST-FNSF would likely be located behind outboard blanket modules and/or the main vacuum vessel, and active RWM-control is envisioned to utilize advanced state-space control techniques [63-65]. Analysis of ideal-wall low-n kink stability for a high- $\beta_{\mathrm{N}}$ ST-FNSF steady-state scenario is described in section 2.4.2, and detailed RWM stability analysis is a topic for future study.

2.1.4. Energy confinement. A key parameter for fusion performance is the plasma thermal energy confinement time $\tau_{\mathrm{E}-\text { th }}$ which relates the thermal stored energy $W_{\text {th }}$ to the plasma heating power $P_{\text {heat }}$ via $W_{\text {th }}=P_{\text {heat }} \tau_{\mathrm{E}-\text { th }}$ [66]. Confinement scalings are a common means of estimating energy confinement, and a widely used scaling is the ITER ELMy H-mode scaling termed ITER IPB98 $(y, 2)[66,67]$. Further, it is common to normalize experimental or expected confinement with respect to the scaling, and this ratio is the confinement multiplier $H_{98}$. A key scientific goal of the NSTX Upgrade research facility and program [27] is to determine if ST confinement scales similarly to conventional aspect ratio or more closely follows confinement scaling on present ST devices [68-71] as the plasma temperature is increased. In terms of dimensionless 'physics' scaling [72, 73], a key question is the dependence of the dimensionless confinement on $\beta$ and collisionality $\nu$, i.e. the determination of the exponents in relations of the form $\Omega \tau_{\mathrm{E}} \propto \beta^{-\alpha_{\beta}} \nu^{-\alpha_{\nu}}$ since present ST data exhibits a weak dependence on $\beta\left(\alpha_{\beta} \approx 0\right)$ as does some conventional aspect ratio data $[72,73]$ but exhibits a stronger nearly inverse dependence on collisionality $\left(\alpha_{\nu}=0.7-1\right)$. This potentially favorable collisionality dependence of confinement leads to projected confinement times 1.5-2 $\times$ higher than the ITER scaling $[27,28]$ for NSTX-U, MAST-U, and ST-FNSF. Such confinement enhancements could significantly reduce the device size and/or required heating power to achieve high fusion performance in an FNSF.

On NSTX, $H_{98}$ values of up to 1.2 were accessible for a range of normalized density values spanning Greenwald fraction $[74,75]$ values of $f_{\text {Greenwald }}=0.5-1$ as shown in figure 7 . Another promising confinement regime is the 'enhanced pedestal H-mode' (EPH) [76-78] which has accessed $H_{98}=$ 1.3-1.5 for a range of plasma conditions and $H_{98}$ approaching 2 for several shots. At the present time, the physics of EPH access is not well understood and achieving more reliable access and sustainment will be an important element of the NSTX-U research program. Given the present uncertainty in achieving very high $\mathrm{H}_{98}$, ST-FNSF configurations studied

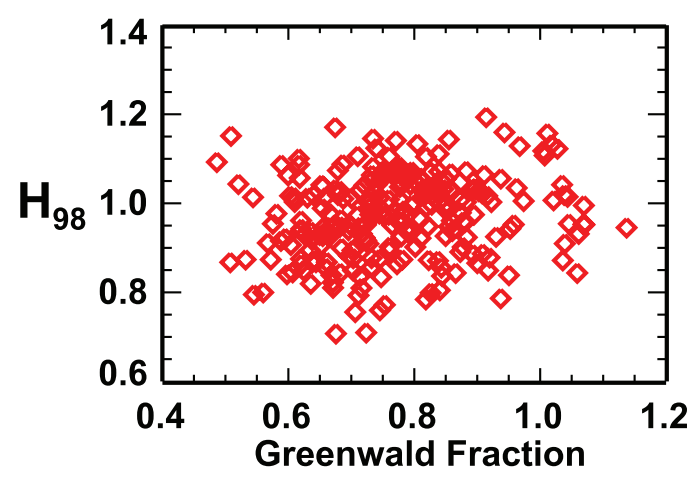

Figure 7. NSTX H-mode thermal confinement multiplier $H_{98}$ relative to the ITER H-98(y,2) confinement scaling versus Greenwald density fraction (figure reproduced with permission [27]).

here aim to achieve FNS baseline mission objectives while only requiring confinement at the level of $H_{98} \leqslant 1.3$.

2.1.5. Heating and current drive. A very important consideration for ST-FNSF is the choice of heating and current drive source. For the typically over-dense plasma conditions of the ST, commonly used RF schemes such as electron cyclotron current drive (ECCD) and lower hybrid current drive (LHCD) suffer from accessibility problems. The high-harmonic fast wave (HHFW) [79, 80] does not suffer from such accessibility problems and has heated plasmas to record ST electron temperatures $T_{\mathrm{e}}(0) \geqslant 6 \mathrm{keV}$ in NSTX [81]. However, due in part to larger trapped particle fraction at low aspect ratio, the current drive efficiency for bulk current drive is relatively low $[27,82,83]$. Further, edge losses in the scrape-off-layer to the divertor [84-87] can degrade HHFW core coupling efficiency, and parasitic absorption by NBI fast-ions [88-90] can compete with thermal electron heating and current drive. For these reasons, HHFW appears most applicable to heating and driving current in low-current high-bootstrap-fraction plasmas [91] serving as targets for subsequent non-inductive current rampup through other means. Electron Bernstein waves (EBW) are another potentially very attractive wave for heating and driving current in over-dense plasma conditions [92-94], but the necessity for precise tailoring of the edge density gradient [95] to maximize the double-mode-conversion efficiency combined with other loss mechanisms in the plasma edge $[96,97]$ have thus far made efficiently coupling to the EBW operationally challenging.

Neutral beam injection (NBI) is one of the few viable options for ST heating and current drive, and nearly all of the present high-performance ST physics basis has been developed using NBI heating. Further, momentum injection will be very important for providing rotation shear to suppress ion turbulence [98-100] to achieve high ion temperatures for fusion applications, and tangentially injected NBI can provide such rotation and rotation shear. The NBI energy and injection tangency radius are critical parameters for optimizing current drive efficiency and the driven current profile. The neutral beam energy must be sufficiently high to penetrate into the plasma core but be nearly fully ionized by the plasma too avoid excessive shine-through and excessive heating of 
plasma facing components where NBI intercepts the first wall. For NBI energies between $0.1-1 \mathrm{MeV}$, the exponential decay length for beam ionization can be approximated as $\lambda_{\text {ionize }}(\mathrm{m}) \approx 5.5 E\left(\mathrm{MeV} \mathrm{amu}^{-1}\right) / n_{\mathrm{e}}\left(10^{20} \mathrm{~m}^{-3}\right)$ [101], and this relation provides a useful guide for estimating the maximum beam energy applicable to a given ST size. In particular, this relation indicates deuterium beams in the range of $0.1-1 \mathrm{MeV}$ are well matched to ST device sizes in the range of $R_{0}=1-2 \mathrm{~m}$ and electron densities of a few $10^{20} \mathrm{~m}^{-3}$. The tangency radius of injection is also important, and injecting at radii larger than the radius of the geometric center of the plasma (i.e. $R_{\text {tang }} / R_{0} \geqslant 1$ ) can provide substantially higher current drive than more perpendicular injection [27]. The maximum tangency radius is limited by the available space between the outboard toroidal field coils and by the required path-length through the plasma for beam ionization and absorption (i.e. the shine-through limit).

The neutralization efficiency for $\mathrm{H}^{+}$or $\mathrm{D}^{+}$ions for positive NBI (PNBI) drops rapidly above $60-75 \mathrm{keV} \mathrm{amu}^{-1}$, and thus the maximum practical injection energy for $D^{0}$ using PNBI is $120-150 \mathrm{keV}[101,102]$. Long-pulse PNBI using the TFTR/NSTX/NSTX-U style sources [27] as proposed for the Tokamak Physics eXperiment (TPX) [103] could be utilized for initial operation of ST-FNSF but would require the development of steady-state neutralization methods. For higher energy injection, Negative NBI (NNBI) $[104,105]$ gas neutralization efficiency remains relatively high at $60 \%$ approximately independent of energy [102]. Substantial technical development of NNBI at $0.5 \mathrm{MeV}$ has been successfully carried out in preparation for JT-60 super advanced (JT-60SA) [106-108] operation and for $1 \mathrm{MeV}$ NNBI for ITER [102, 109, 110]. For the purposes of this paper, it is assumed that NNBI suitable for ST-FNSF will be developed through a combination on ongoing research and development for JT-60SA and ITER and that steady-state operation and higher electrical efficiency could eventually be achieved through improved plasma and/or photo-neutralization [102, 111].

For the OD scaling calculations performed here, the NBI current drive efficiency is estimated using equations (44) and (45) of [112] which include the effects of fast-ion slowing down and simplified tokamak geometry effects and are applicable to arbitrary injection energy and thermal species temperatures. The leading order scaling of the current drive efficiency is proportional to the well-known $P_{\mathrm{NBI}} T_{\mathrm{e}}\left(1-Z_{\text {beam }} / Z_{\mathrm{eff}}\right) / n_{\mathrm{e}} R$ dependence $[113,114]$, and the computed efficiency from [112] is rescaled by a factor 0.7 to approximately account for field-line pitch and profile effects which reduce the efficiency, and to improve agreement with TRANSP NBI current drive calculations both for the FNSF calculations here and for experimentally inferred values in NSTX [34, 115] when core MHD activity was sufficiently weak. The fast-ion stored energies for NBI/alpha particles are computed analytically by calculating the fast-particle injection/birth rates, thermalization times, and average energies during thermalization. The fast-ion and thermal stored energies are then used to compute the total stored energy, $\beta_{\mathrm{N}}$, and $\beta_{\mathrm{T}}$ values. Fusion powers and neutron rates are computed from scalings for thermonuclear and beam-target [116] reactions (beam-beam reactions are ignored) cross-checked with TRANSP calculations.

Lastly, the bootstrap current fraction [117-119] $f_{\mathrm{BS}}=$ $I_{\mathrm{BS}} / I_{\mathrm{P}}$ is assumed to scale as $C_{\mathrm{BS}} \sqrt{\epsilon} \beta_{\mathrm{pol}-\mathrm{th}}$. Here $\beta_{\mathrm{pol}-\text { th }}$ is the poloidal beta as defined in [17] using the thermal pressure. The bootstrap fraction coefficient is assumed to vary as $C_{\mathrm{BS}}=\operatorname{MAX}\left(1.2-0.2 \times q^{*} / q_{\min }, 0.6\right)$ similar in form to the ITER scaling described in [119] but consistent with lowaspect-ratio equilibrium calculations [17, 20, 27]. The total non-inductive current for a given scenario is then calculated from the sum of the bootstrap and NBI currents. All these calculations are included in a zero-dimensional systemscode written for this paper to compute non-inductive current drive from the NBI and bootstrap currents for fully noninductive plasmas while also computing a wide range of other fusion performance parameters.

\subsubsection{Fusion performance dependence on density and}

size. A fundamental requirement for an FNSF is achievement of neutron wall loading of at least $1 \mathrm{MW} \mathrm{m}^{-2}$ while providing sufficient component testing area $\geqslant 10 \mathrm{~m}^{2}$ [11]. Based on previous design studies and calculations performed here, the lower-bound on device size capable of meeting these neutron flux and testing area goals is $R_{0} \approx 1 \mathrm{~m}$. The ST pilot plant [3] provides an upper-bound on major radius with $R_{0}=2.2 \mathrm{~m}$ for a device that can achieve both the FNSF mission and small net electricity production. This range of major radii $R_{0}=1-2.2 \mathrm{~m}$ is studied in the remainder of this paper. For the purposes of configuration comparison, the plasma-surface-average neutron flux $\left\langle\Gamma_{\mathrm{n}}\right\rangle$ is typically held fixed at $1 \mathrm{MW} \mathrm{m}^{-2}$ as a constraint. This flux is approximately equal to the average neutron wall loading $\left\langle W_{\mathrm{n}}\right\rangle$ provided the plasma-wall gap is small relative to the plasma minor radius, and unless otherwise stated, these parameters are used interchangeably, i.e. $\left\langle\Gamma_{\mathrm{n}}\right\rangle \approx\left\langle W_{\mathrm{n}}\right\rangle$.

Using the physics assumptions described in previous sections, a device major radius half way between the minimum $\left(R_{0}=1 \mathrm{~m}\right)$ and maximum $\left(R_{0}=2.2 \mathrm{~m}\right)$ values of this study is chosen (i.e. $R_{0}=1.6 \mathrm{~m}$ ) to investigate the impact of varied plasma density. Assuming fixed aspect ratio $A=1.7$, elongation $\kappa=2.75, B_{\mathrm{T}}=3 \mathrm{~T}$, NNBI injection energy $E=0.5 \mathrm{MeV}$ for heating and current drive, and ITER H-mode confinement multiplier $H_{98}=1.25$, it is found that $80 \mathrm{MW}$ of NNBI heating power is required to achieve $\left\langle W_{\mathrm{n}}\right\rangle \geqslant 1 \mathrm{MW} \mathrm{m}^{-2}$ for the range of normalized densities $f_{\text {Greenwald }}=0.5-1$ considered. Figure $8(a)$ shows that for this normalized density range $\beta_{\mathrm{N}}=4-4.7, q^{*} \geqslant 3, Q_{\mathrm{DT}}=1.8-2.7$, and $\left\langle W_{\mathrm{n}}\right\rangle=$ 1-1.5 MW $\mathrm{m}^{-2}$. Figure $8(b)$ shows the plasma current $I_{\mathrm{P}}=10.8-12 \mathrm{MA}, \beta_{\mathrm{T}}=16-19 \%$, and the fusion power $P_{\text {fusion }}$ varies from 146-220 MW with the highest plasma densities resulting in the highest fusion power and gain and neutron wall loading. The neutron wall loading is primarily from thermonuclear fusion reactions with beam-target neutron power fractions (not shown) of $27 \%, 18.5 \%$, and $11 \%$ at $f_{\text {Greenwald }}=0.5$, 0.75 , and 1 , respectively.

Stronger dependences on plasma density are observed for other operating parameters as shown in figure 9. Figure 9(a) shows that the bootstrap current fraction $f_{\mathrm{BS}}$ increases from 

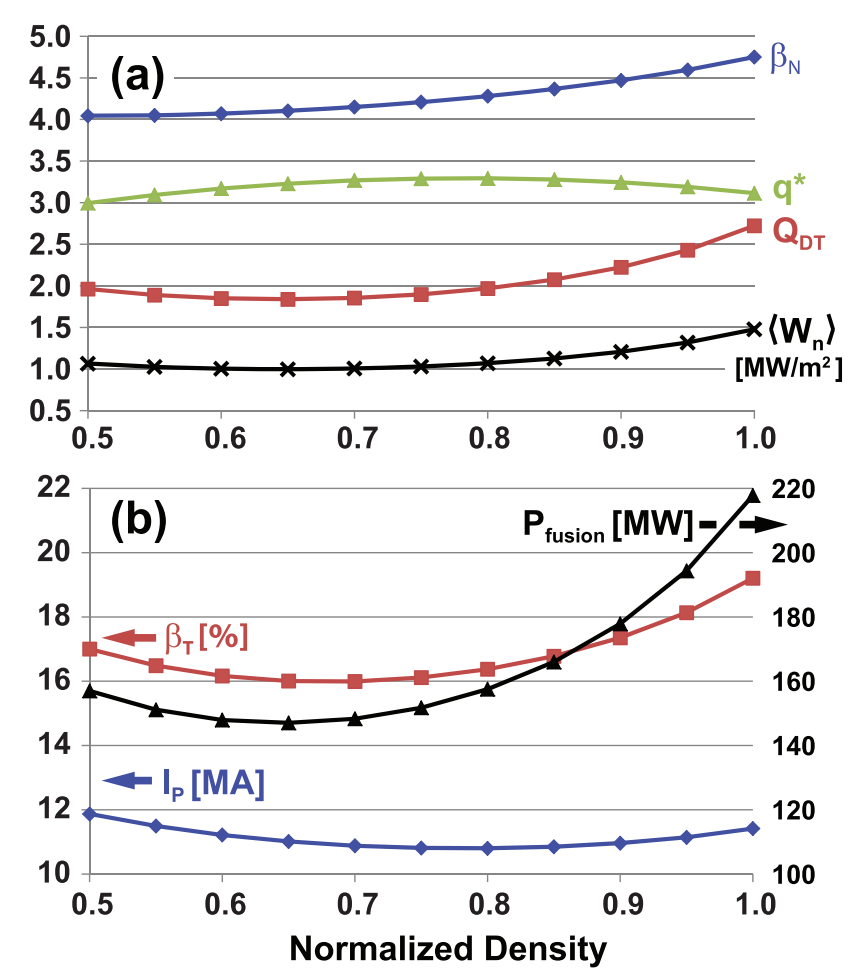

Figure 8. ST-FNSF parameters versus normalized density at fixed major radius $R_{0}=1.6 \mathrm{~m}$ and NBI heating power $P_{\mathrm{NBI}}=80 \mathrm{MW}$.

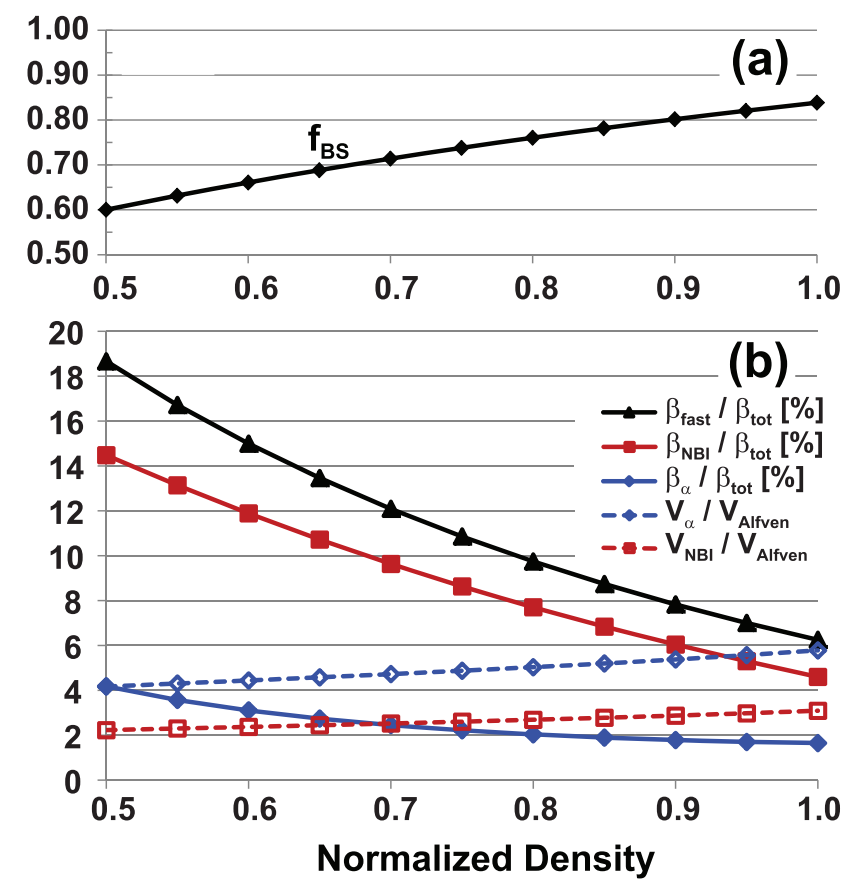

Figure 9. ST-FNSF $(a)$ bootstrap current fraction and $(b)$ fast-ion parameters versus normalized density at fixed major radius $R_{0}=1.6 \mathrm{~m}$ and $\mathrm{NBI}$ heating power $P_{\mathrm{NBI}}=80 \mathrm{MW}$.

60-84\% which implies the beam-driven current drive fraction decreases by a factor of 2.5 from $40-16 \%$ as $f_{\text {Greenwald }}$ is increased from 0.5 to 1 . Similarly, figure $9(b)$ shows the fast-ion beta fraction $\beta_{\text {fast }} / \beta_{\text {total }}$ decreases by a factor of 3 from 18 to $6 \%$ due (in part) to reduced fast-ion slowing down time resulting from lower average electron temperature at higher density. As shown in the same figure, the D-T fusion alpha-particle contribution is $20-30 \%$ of the total fast-ion pressure and the remainder comes from NBI fast-ions. Defining an Alfven speed based on the vacuum field $B_{\mathrm{T}}$ at the plasmageometric-center and the line-average main-ion density, both species of fast ions are computed to be super-Alfvenic with $V_{\alpha} / V_{\mathrm{Alfven}}=4-6$ and $V_{\mathrm{NBI}} / V_{\mathrm{Alfven}}=2-3$. As demonstrated in NSTX, instabilities driven by super-Alfvenic fast-ions of sufficiently high $\beta$ fraction can lead to fast ion redistribution and/ or loss and can significantly impact the NBI-driven current [115]. This physics will be explored at a more FNSF-relevant higher toroidal field and instability toroidal mode-number in NSTX-U in which stronger mode damping will compete with stronger instability drive relative to NSTX plasmas [120].

Recent parametric studies of NSTX plasmas indicate that fast-ion instabilities can be largely avoided even at high $V_{\text {fast }} / V_{\text {Alfven }} \leqslant 6$ if the fast-ion $\beta$ fraction is below approximately $10 \%$ [121]. Further, maintaining sufficiently low fast-ion fraction may also play an important role in retaining elevated no-wall and ideal-wall mode stability limits close to lower-rotation and thermal-plasma values [47]. However, it is also advantageous from a plasma control standpoint to maximize the non-inductive current drive fraction from neutral beams to have more control over the $q$ profile and associated confinement and stability properties of the plasma. For these reasons, a normalized density $f_{\text {Greenwald }} \approx 0.8$ appears favorable for simultaneously accessing $\beta_{\text {fast }} / \beta_{\text {total }} \leqslant 10 \%$ and providing a NBI-CD fraction of $\geqslant 20 \%$ while also staying below the nominal density limit of $f_{\text {Greenwald }} \approx 1$.

Figure 10 shows several performance parameters as a function of device major radius $R_{0}$ varied from $1 \mathrm{~m}$ to $2.2 \mathrm{~m}$ at fixed $f_{\text {Greenwald }}=0.8$ and surface-average neutron wall loading slightly above $1 \mathrm{MW} \mathrm{m}^{-2}$. The beam energy, aspect ratio, field, and confinement are fixed at $E_{\mathrm{NBI}}=0.5 \mathrm{MeV}$, $A=1.7, B_{\mathrm{T}}=3 \mathrm{~T}$, and $H_{98}=1.25$, respectively. As shown in figure $10(a)$, the increase in major radius tends to be moderately stabilizing as $\beta_{\mathrm{N}}=4.9 \rightarrow 4$ and $q *=3 \rightarrow 3.5$ while the fusion gain increases from $Q_{\mathrm{DT}}=1 \rightarrow 3.2$. As shown in figure $10(b)$, the toroidal beta decreases moderately from $\beta_{\mathrm{T}}=18 \% \rightarrow 15 \%$. The plasma parameters that vary most strongly with major radius are the plasma current and fusion power. As shown in figure $10(b), I_{\mathrm{P}}$ increases by approximately a factor of 2 from $7 \mathrm{MA} \rightarrow 14 \mathrm{MA}$, and as shown in figure $10(c)$, the fusion power increases $60 \mathrm{MW} \rightarrow 300 \mathrm{MW}$ as the heating power increases from $60 \mathrm{MW} \rightarrow 95 \mathrm{MW}$. Figure $11(a)$ shows that the bootstrap fraction $f_{\mathrm{BS}}$ decreases from $81 \% \rightarrow 72 \%$, and figure $11(b)$ shows that $\beta_{\text {fast }} / \beta_{\text {tot }}$ increases only $16 \%$ from $8.8 \% \rightarrow 10.2 \%$ as $\beta_{\alpha} / \beta_{\text {tot }}$ increases from $1 \%$ to $3 \%$. Both $V_{\alpha} / V_{\text {Alfven }}$ and $V_{\mathrm{NBI}} / V_{\text {Alfven }}$ decrease by $40 \%$ as the major radius is increased which may imply reduced drive for fast-ion instabilities at larger machine size. It should also be noted that NNBI at $E=0.5 \mathrm{MeV}$ may be too energetic to be fully absorbed at large tangency radius in smaller FNSF devices, and if lower energy beams are used, more power may be required (due to reduced current drive efficiency) to achieve the assumed NBI current drive. From these size scans it is concluded that the overall impact of increased major radius on normalized plasma parameters is relatively modest. However, since the neutron wall loading is 

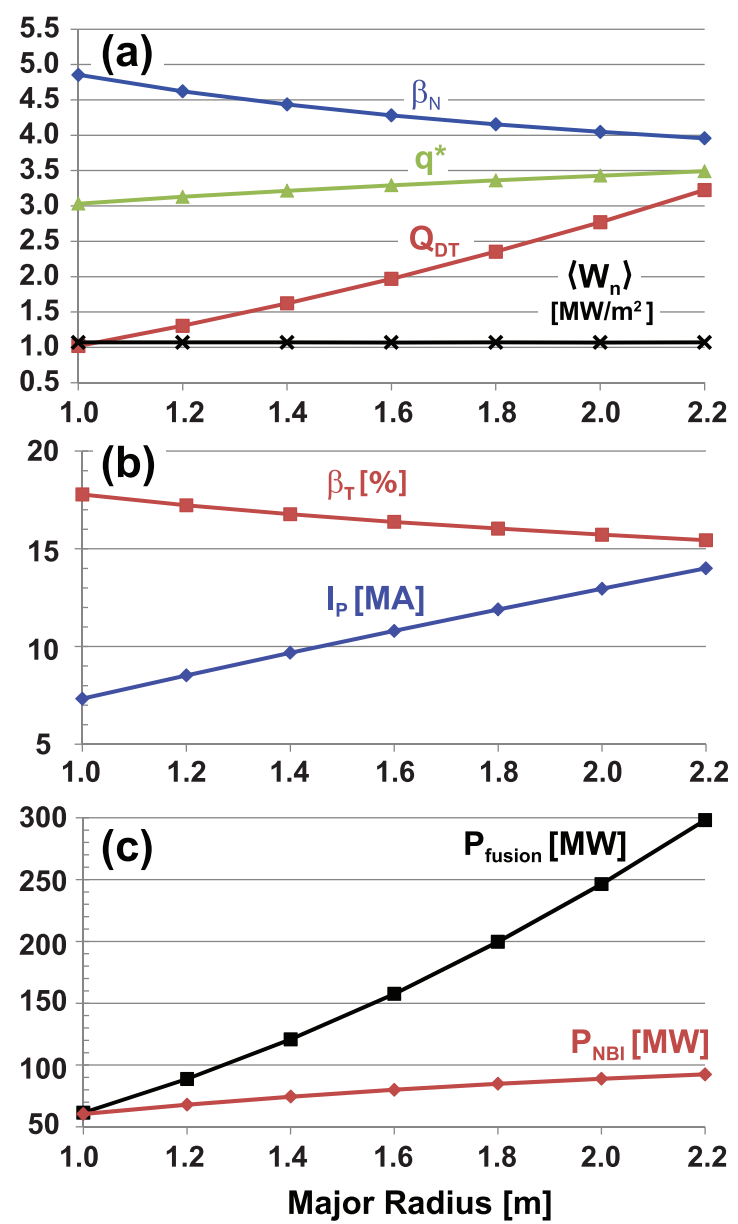

Figure 10. ST-FNSF parameters versus device major radius at fixed average neutron wall loading $=1.1 \mathrm{MW} \mathrm{m}^{-2}$.
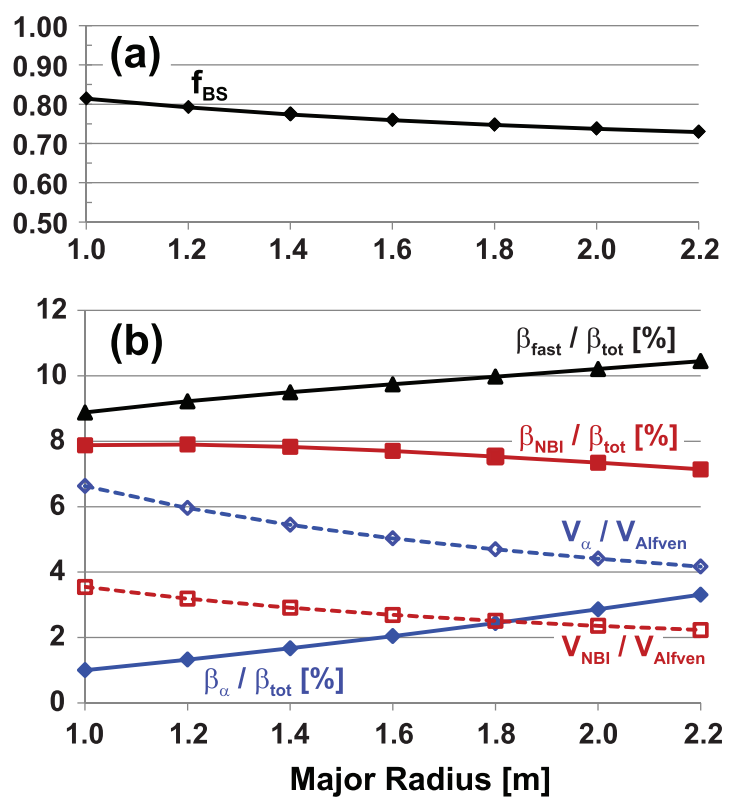

Figure 11. ST-FNSF $(a)$ bootstrap current fraction and $(b)$ fast-ion parameters versus device major radius at fixed average neutron wall loading $=1 \mathrm{MW} \mathrm{m}^{-2}$.

held fixed, the fusion power and tritium consumption scale as the surface area $\propto R^{2}$ and increase by a factor of 5 as the major radius is increased from $R_{0}=1 \mathrm{~m}$ to $2.2 \mathrm{~m}$.

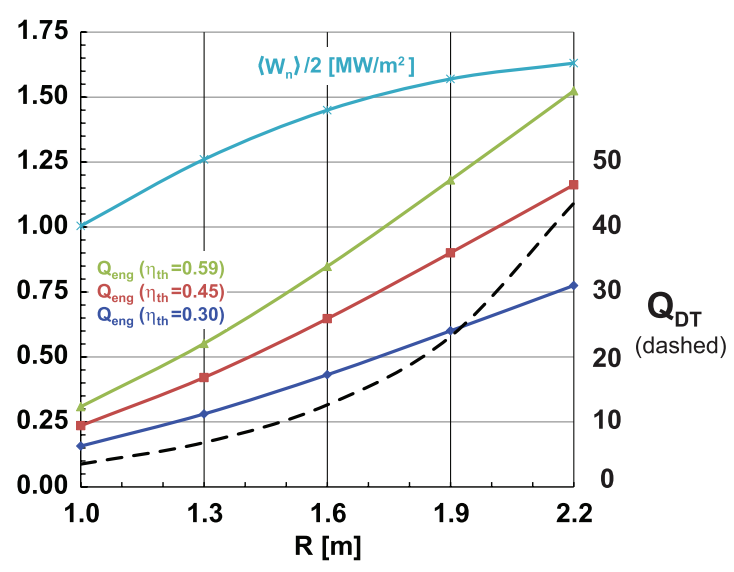

Figure 12. Engineering and fusion gains and neutron wall loading versus device size for high-performance ST-FNSF scenarios.

In the limit where tritium self-sufficiency is not required, clearly small STs are favored since they minimize fusion power and tritium consumption. However, tritium self-sufficiency and electrical self-sufficiency are ultimately required for the development of fusion energy, so it is important to determine the thresholds in device size for achieving these goals. Initial assessments of tritium self-sufficiency are described in section 4.2. For electrical self-sufficiency, the engineering efficiency $Q_{\text {eng }}$ (utilizing the same parametric assumptions as in previous pilot plant studies [3]) is defined as the ratio of electrical power produced $P_{\text {elec }}$ to electrical power consumed and can be expressed as:

$Q_{\text {eng }}=\frac{\eta_{\text {th }} \eta_{\text {aux }} Q\left(4 M_{\mathrm{n}}+1+5 / Q+5 P_{\text {pump }} / P_{\text {fus }}\right)}{5\left(1+\eta_{\text {aux }} Q\left(P_{\text {pump }}+P_{\text {sub }}+P_{\text {coils }}+P_{\text {control }}\right) / P_{\text {fus }}\right)}$

where $\eta_{\mathrm{th}}=$ thermal conversion efficiency $=P_{\text {elec }} / P_{\mathrm{th}}, \eta_{\mathrm{aux}}=$ auxiliary power wall plug efficiency, $P_{\text {fus }}=$ total D-T fusion power, $P_{\mathrm{aux}}=$ auxiliary power for heating and current-drive, $Q=P_{\text {fus }} / P_{\text {aux }}, M_{\mathrm{n}}=$ neutron energy multiplication factor, $P_{\mathrm{n}}$ and $P_{\alpha}=$ neutron and alpha powers from fusion, $P_{\mathrm{th}}=$ thermal power $=M_{\mathrm{n}} P_{\mathrm{n}}+P_{\alpha}+P_{\mathrm{aux}}$, $P_{\text {pump }}=$ coolant pumping power, $P_{\text {sub }}=$ subsystems power, $P_{\text {coils }}=$ power dissipated in normally conducting coils, and $P_{\text {control }}=$ power used in plasma or plant control not included in $P_{\text {aux }}$. Equation (1) illustrates that the leading terms in the engineering efficiency $Q_{\text {eng }}$ involve a combination of technology and physics performance metrics. In particular, $Q_{\text {eng }}$ depends to leading order on the thermal conversion and auxiliary system wall-plug efficiencies $\left(\eta_{\text {th }}\right.$ and $\left.\eta_{\text {aux }}\right)$ and the D-T fusion gain $Q$. To achieve electrical self-sufficiency in the modest-sized ST devices considered here requires high blanket thermal conversion efficiency and increased confinement and stability. For this analysis, the value of $\eta_{\text {th }}$ is varied to assess the impact on $Q_{\text {eng }}$, a constant $\eta_{\text {aux }}=0.4$ (higher than presently achievable [3]) and $M_{\mathrm{n}}=1.1$ are assumed. $E_{\mathrm{NBI}}=0.5 \mathrm{MeV} \mathrm{NNBI}$ is assumed for heating and current drive resulting in a normalized current drive (CD) efficiency $\eta_{\mathrm{CD}} \equiv I_{\mathrm{CD}} R_{0} n_{\mathrm{e}} / P_{\mathrm{CD}} T_{\mathrm{e}} \approx 0.04 \times 10^{20} \mathrm{~A} \mathrm{Wm}{ }^{-2} \mathrm{keV}$.

Figure 12 shows the surface-average neutron flux, engineering efficiency, and fusion gain $Q=Q_{\mathrm{DT}}$ for a range of blanket thermal conversion efficiencies and device sizes 

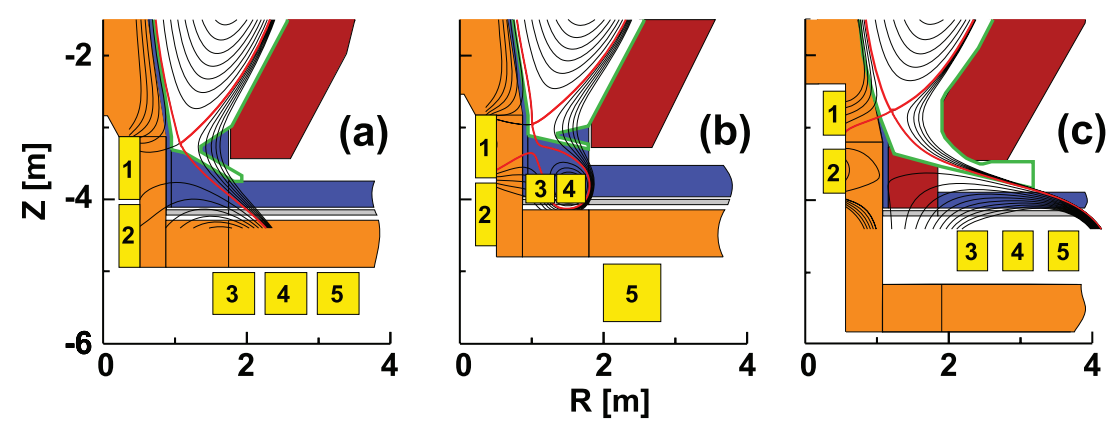

Figure 13. Divertor-region cross-sections for three $R_{0}=1.7 \mathrm{~m}$ ST-FNSF divertor configurations: $(a)$ conventional, $(b)$ snowflake, and (c) super-X divertor.

for high-performance ST-FNSF scenarios targeting net electricity production. These scenarios have fixed $A=1.7$, $B_{\mathrm{T}}=3 \mathrm{~T}, H_{98}=1.5, \beta_{\mathrm{N}}=5.5, \kappa=3.0$, and $f_{\text {Greenwald }}=0.8$ resulting in $\beta_{\mathrm{T}}=25-28 \%$ and $q^{*} \approx 3$. Such scenarios have normalized confinement and stability performance near or at the highest values achieved on NSTX. Importantly, figure 12 shows that as $R_{0}$ is increased from $1 \mathrm{~m}$ to $2.2 \mathrm{~m}$ the smallest possible ST device that can achieve electricity break-even $\left(Q_{\text {eng }} \approx 1\right)$ has $R_{0}=1.75 \mathrm{~m}$ assuming very high blanket thermal conversion efficiency $\eta_{\text {th }}=0.59$ as used in the ARIES-AT power plant design [122]. For $\eta_{\text {th }}=0.45$, the required device size to achieve $Q_{\mathrm{eng}}=1$ increases to $R_{0}=$ $2 \mathrm{~m}$, and still larger devices are required for lower $\eta_{\mathrm{th}}$. The fusion power is 2-3 times higher and NBI auxiliary heating power 2-3 times lower for these higher performance scenarios as compared to the cases shown in figure 10 where $H_{98}=1.25$ is assumed. As shown in figure 12, this results in $Q_{\mathrm{DT}}$ increasing from $4 \rightarrow 44$ as $R_{0}=1 \mathrm{~m} \rightarrow 2.2 \mathrm{~m}$. Such a device would also have a relatively high surface-average neutron flux in the range of $2-3 \mathrm{MW} \mathrm{m}^{-2}$. From these studies it can be concluded that a normally-conducting toroidal field coil ST-FNSF device with $R_{0} \geqslant 1.75 \mathrm{~m}$ could hypothetically achieve $Q_{\text {eng }} \geqslant 1$ but would require both advanced physics and engineering performance.

\subsection{Free-boundary equilibrium calculations}

Achieving high elongation $\kappa$ and triangularity $\delta$ can be challenging in the ST configuration since at least one set of divertor poloidal field (PF) coils is required to be both inboard and close to the divertor $\mathrm{x}$-point. This is challenging in a nuclear environment since neutron damage to the PF coil insulation can substantially reduce the lifetime of the insulator and hence the coil. Figure 13 shows a potential solution in which two PF coils (labeled 1 and 2) are installed at the ends of the TF central rod in a Bitter plate configuration using $\mathrm{MgO}$ insulation (see section 3.1 for design details). For these coils, the CS shield and TF Cu conductor help shield the PF coils. Freeboundary equilibrium calculations show that such PF coils at the ends of the TF can provide high triangularity $=0.5-0.6$, and that this is sufficient to provide shaping factor $S=25-30$ for $q^{*}=3-4$ for FNSF scenarios. With the inboard PF1 and 2 coils incorporated, additional PF coils at the top and bottom of the device can be added to optimize the divertor configuration for power and particle exhaust.
2.2.1. Divertor configurations. A range of divertor configurations have been studied for ST-FNSF [123] as shown in figure 13 ranging from (a) conventional, to (b) snowflake/X [124, 125] and (c) long-leg/super-X [12, 126-128]. For each of the cases shown, the angle of incidence of the total B-field at the strike-point is constrained to be $\theta_{\mathrm{B}}=1-1.5^{\circ}$ [129]. In figure 13 the PF coils are colored yellow, the TF conductor orange, shielding/vessel blue, blanket red, and limiter outline green. For the conventional divertor, the strike-point is placed close to a diagonal exhaust slot for pumping and the divertor exhaust control coils PF3, 4, 5 are placed outside the TF coil in an effort to reduce the TF power consumption by reducing the overall height of the $\mathrm{Cu}$ TF conductors in the centerstack. Alternatively, if the TF coils were placed outside the PF3-5 coils, additional in-vessel space could be utilized for a vertical target divertor $[130,131]$. To achieve a snowflake divertor with the secondary x-point nearly or fully overlapping with the primary $\mathrm{x}$-point while simultaneously providing high triangularity, PF coils 3-4 must be brought closer to PF coils 1-2 as shown in figure 13(b). The poloidal flux expansion for this configuration is large (40-60) and facilitates detachment and significant heat flux reduction (up to a factor 7) as demonstrated in NSTX [132] and as planned to be tested at high power density in NSTX-U [27]. Concepts for poloidal field coils 3 and 4 using $\mathrm{Cu}$ conductor, $\mathrm{MgO}$ insulation, and $\mathrm{WC}$ shielding would marginally be able to provide the necessary coil current, but for such coils there would be no option to make them superconducting (to reduce overall power consumption) due to the excessive nuclear heating and radiation damage (see section 4.1).

Since parallel heat transport dominates cross-field transport in the scrape-off-layer (SOL), significant reduction of the peak perpendicular divertor heat flux can be achieved by reducing $|B|$ at the strikepoint at fixed angle of B-field incidence [127]. Since $|B| \approx\left|B_{\phi}\right|$ in the divertor and $B_{\phi} \propto 1 / R$, increasing the strike-point major radius is a potentially effective means of divertor heat-flux reduction and is a major motivation for longleg and super-X [127] divertor configurations. As is evident from comparing figures $13(b)$ and $(c)$, the major radius of the strike-point can be increased by a factor of 2-3 in the long-leg/ super-X configuration as compared to the 'conventional' and 'snowflake' configurations. Other important changes shown in figure 13(c) include: the PF1 and 2 coils are closer to the midplane, the PF3-5 coils are inside the TF coils to increase proximity to the plasma make the strike-line more horizontal, 
(a)

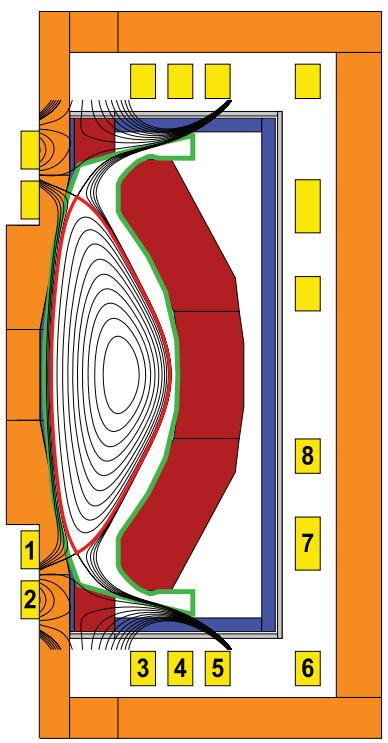

(b)

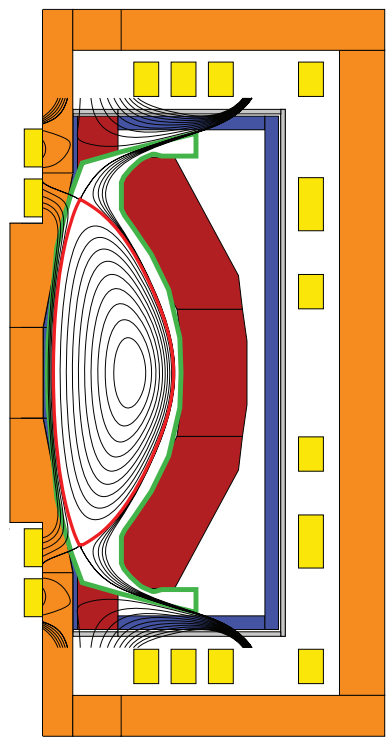

(c)

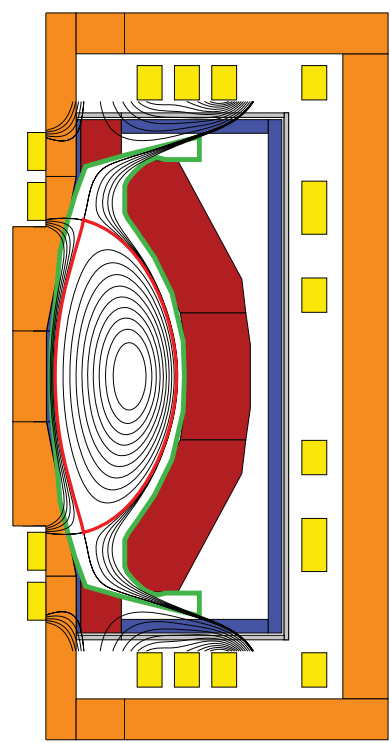

Figure 14. $R_{0}=1.7 \mathrm{~m}$ FNSF device cross-sections for 3 equilibria with $\beta_{\mathrm{N}}=5$ and $(a)$ low $l_{\mathrm{i}}$ and high $\kappa,(b)$ intermediate/reference $l_{\mathrm{i}}$ and $\kappa$, and $(c)$ high $l_{\mathrm{i}}$ and low $\kappa$.

the primary $\mathrm{x}$-point is radially outboard of $\mathrm{PF} 1$, and the secondary $\mathrm{x}$-point is radially outboard of PF2 and the presence of this $\mathrm{x}$-point assists in generating a snowflake-like divertor leg that is significantly closer to horizontal than the strikeline near the primary x-point. The more horizontal exhaust channel aids in increasing the strike-point radius without significantly increasing the overall height of the device. Additional benefits of this increased strike-point radius include reduced neutron flux and fluence at the divertor plasma facing components (PFCs) due to at least partial shielding by the breeding blankets [127]. Further, rather than having a divertor power and particle exhaust region at the top and bottom of the device, this region can be used for breeding which is important for increasing the TBR $>1$ for the ST-FNSF configurations studied here (see section 4.2). The multiple advantages of the long-leg divertor configuration in figure 13(c) motivate adoption of this type of divertor for ST-FNSF.

2.2.2. Equilibrium shape variation. With the adoption of a long-legged divertor for ST-FNSF, the next important step is to assess device flexibility with respect to current profile and pressure variation while retaining acceptable power exhaust configurations. Since predictive capability for electron thermal and ion/electron particle transport have not yet been achieved, there is insufficient theoretical basis for predicting pressure and current profile shapes in ST-FNSF. However, as shown in figure 1, a vast majority of the NSTX data at high elongation was achieved with $l_{\mathrm{i}}=0.4-0.85$, and this range of inductance can be explored as a viable equilibrium range for ST-FNSF.

Figure 14 shows free-boundary equilibrium calculations for a range of inductance and corresponding elongations $\kappa_{x-\mathrm{ST}} \leqslant 3.4-l_{\mathrm{i}}$. For each of the configurations shown, the divertor strike-point radius was maintained near the nominal radius $R_{\text {strike }}=2.5 \mathrm{~m} \pm 0.1 \mathrm{~m}$ and the total $\mathrm{B}$-field angle of incidence maintained at $\theta_{\mathrm{B}}=1-1.5^{\circ}$. The positions of the poloidal field coils (in particular the outboard PF coils 6-8) have been optimized to maintain sufficient inboard and outboard gaps to the blankets and divertors as the current profile and plasma shape are varied while also minimizing the coil current density and staying within allowable limits. There is insufficient space for inboard (high-field-side) slotted divertors in this device configuration for particle control, but a large outboard-to-inboard power exhaust ratio of at least $4: 1$ has been observed in balanced double-null divertor (DND) ST configurations [133-135], and this asymmetry combined with the large poloidal flux expansion near the x-points in the DND should have acceptable inboard peak divertor heat fluxes (see for example projections in figure 20).

Figure 15 shows an overlay of the plasma boundaries for some of the equilibria used to define the limiter and coil positions. As is evident from the figure, the inboard limiter shape is determined primarily by the need for sufficient inboard gap near the $\mathrm{x}$-points of the lowest internal inductance scenario (red boundary). In contrast, the outboard blanket and limiter shapes are determined by the need for sufficient gaps/space for the boundary shape of the highest internal inductance shape (purple boundary) and also the increased poloidal flux expansion in the entrance of the divertor region as evident in figure 14(c). Figure 15 also shows that aside from the elongation change with internal inductance, there is also inboard gap and aspect ratio variation, and more importantly significant variation in the boundary squareness [136].

Figure 16 shows several plasma boundary shaping parameters for a range of internal inductance $l_{\mathrm{i}}$ and $\beta_{\mathrm{N}}$ values. It is not expected that $\beta_{\mathrm{N}}=8$ equilibria can be stably accessed in ST-FNSF, but this high value of $\beta_{\mathrm{N}}$ is useful for equilibrium scoping and PF coil specification. Figure 16(a) shows that the aspect ratio $A$ depends primarily on $l_{\mathrm{i}}$, and $A$ decreases with increasing $l_{\mathrm{i}}$ as the inboard gap shrinks with a more peaked 


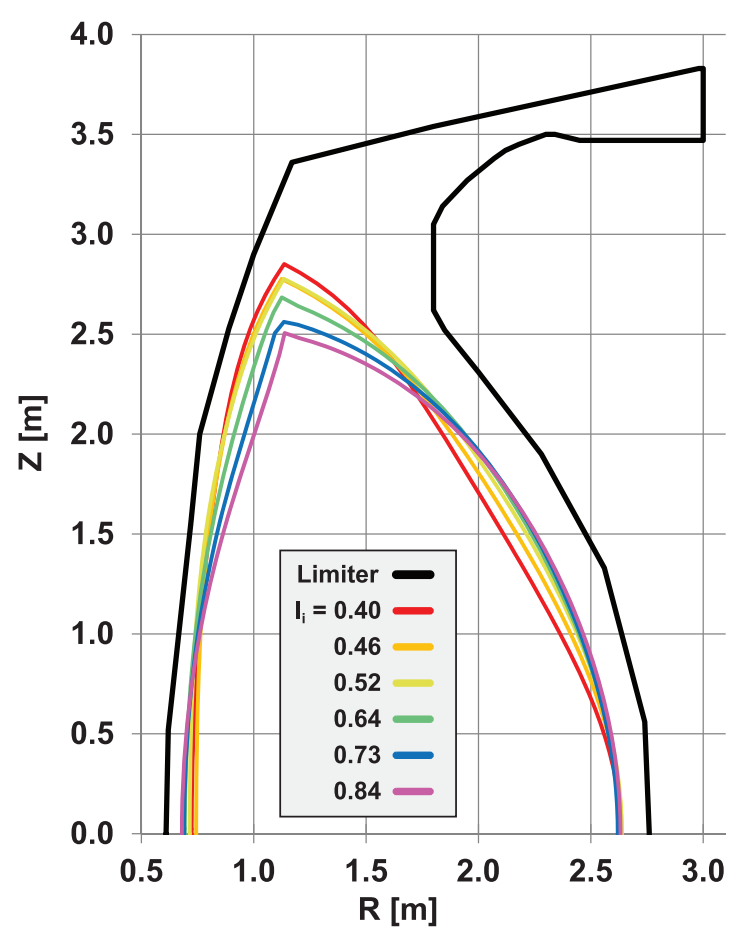

Figure 15. Overlay of limiter surface (black) and free-boundary $\beta_{\mathrm{N}}=5$ equilibrium plasma boundaries for varied internal inductance $l_{\mathrm{i}}$ for $R_{0}=1.7 \mathrm{~m} \mathrm{ST}-F N S F$.

current profile and lower elongation. Figure $16(b)$ shows that all equilibria approximately follow the prescribed scaling of X-point elongation $\kappa_{x}$ with $l_{\mathrm{i}}$ shown by the dashed black line. Figure $16(c)$ shows that for the higher $\beta_{\mathrm{N}}$ scenarios the $\mathrm{x}$-point triangularity $\delta_{x}=0.5-0.65$ and is nearly independent of $l_{\mathrm{i}}$ for $l_{\mathrm{i}} \leqslant 0.7$, while for low $\beta_{\mathrm{N}}$ the achievable $\delta_{x}=0.45-0.5$ is lower and transitions to the lowest values for $l_{\mathrm{i}} \geqslant 0.6$. Figure $16(d)$ shows the boundary outer squareness increases nearly linearly from $\zeta_{o}=-0.15$ to 0.0 over the $l_{\mathrm{i}}$ range $0.4-0.7$ and plateaus at $\zeta_{o}=0-0.05$ for $l_{\mathrm{i}} \geqslant 0.7$.

2.2.3. Safety factor, poloidal field coil currents. As described in section 2.1.3, maintaining sufficiently high safety factor is important for avoiding the onset of current-driven kink modes and increased disruptivity. Increased $l_{\mathrm{i}}$ reduces the maximum $\kappa$ and $q^{*}$ as shown in figure 17(a) for the reference $R_{0}=1.7 \mathrm{~m}$ equilibrium with $I_{\mathrm{P}}=11.6 \mathrm{MA}$. As is evident from this figure, maintaining $q^{*} \geqslant 3$ requires maintaining $l_{\mathrm{i}} \leqslant 0.7$ and the lower $\beta_{\mathrm{N}}$ scenarios have slightly higher $q^{*}$ due to slightly lower aspect ratio. Figure $17(b)$ shows a similar trend of decreasing $q_{95}$ with increasing $l_{\text {i }}$ with a minimum $q_{95}$ value of 4.5 for the highest $l_{\mathrm{i}}$ studied. Just as there are current and current density limits for the plasma current channel, there are also current and current density limits on the poloidal field coils maintaining the tokamak equilibrium. Figure 17(c) shows that the total divertor PF coil current (PF1 + PF2) is in the range of 8-12 MA which is comparable in magnitude to the reference $I_{\mathrm{P}}=11.6 \mathrm{MA}$. The highest divertor coil current is needed for the lowest $\beta_{\mathrm{N}}$ and highest $l_{\mathrm{i}}$ values. In contrast, figure $17(d)$ shows that the primary outboard vertical field coils (PF7 + PF8) have the highest magnitude (most negative) current for the highest $\beta_{\mathrm{N}}$ and lowest $l_{\mathrm{i}}$ values.
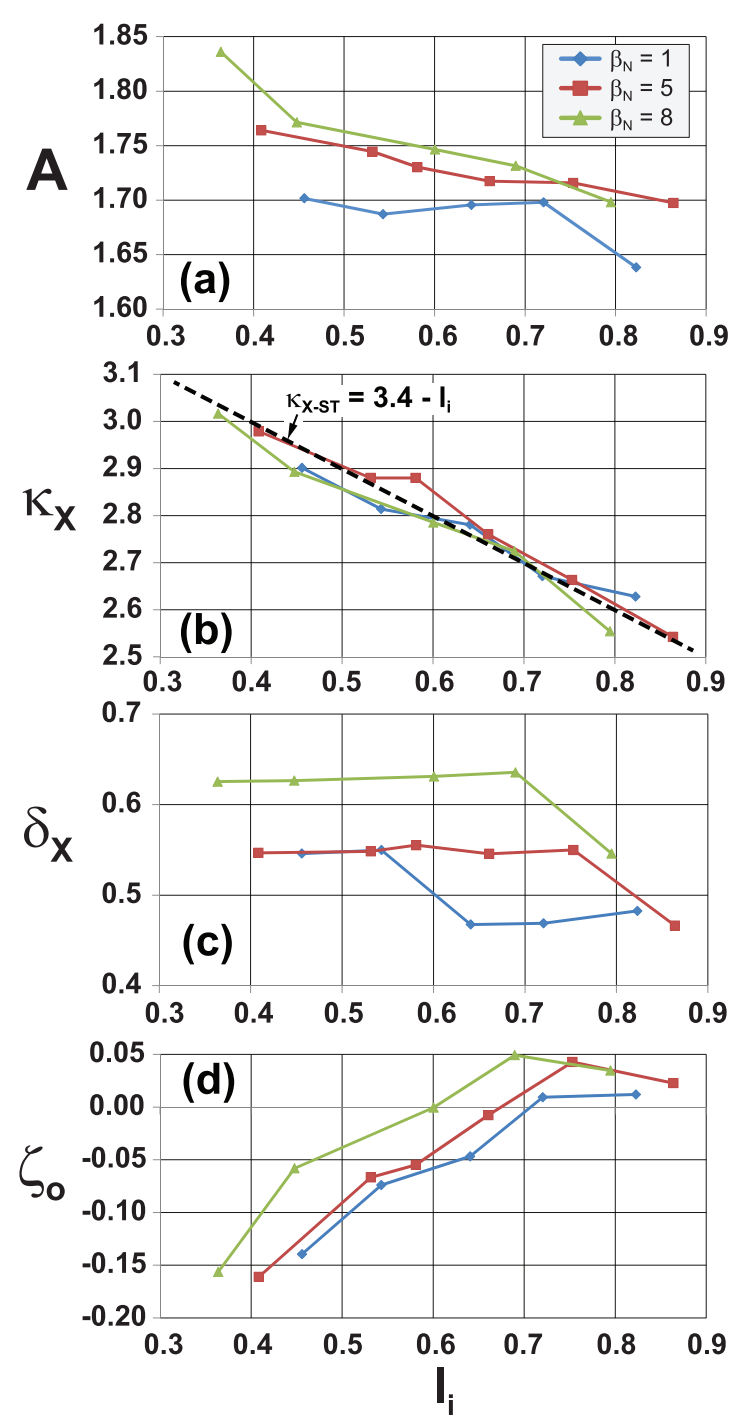

Figure 16. Plasma boundary ( $a$ ) aspect ratio $A,(b)$ x-point elongation $\kappa_{X},(c)$ x-point triangularity $\delta_{X}$, and $(d)$ outboard squareness $\zeta_{o}$ for a range of $l_{\mathrm{i}}$ and $\beta_{\mathrm{N}}$ for $R_{0}=1.7 \mathrm{~m} \mathrm{ST-FNSF}$.

For the PF coils considered for ST-FNSF, thermalhydraulic analysis finds the estimated winding-pack current density limits for multi-turn water-cooled copper coils using $\mathrm{MgO}$ insulation and stainless steel jacketing is 4-10 $\mathrm{MA} \mathrm{m}^{-2}$ $[137,138]$, while Bitter plate magnets have been operated at very high current densities up to $400 \mathrm{MA} \mathrm{m}^{-2}$ [139] in environments where radiation resistance is not an issue. Incorporating a ceramic radiation-resistant insulator may degrade this current density value somewhat, and research will be needed to verify that radiation will not induce arcing through the magnet cooling water [140]. Approximate winding-pack current density limits for low-temperature-superconductor (LTS) cablein-conduit conductor (CICC) [141] coils are $14 \mathrm{MA} \mathrm{m}^{-2}$ for ITER NbTi PF coils $[142,143], 18 \mathrm{MA} \mathrm{m}^{-2}$ for $1000 \mathrm{~A} \mathrm{~mm}^{-2}$ critical current density $\mathrm{Nb}_{3} \mathrm{Sn}$ strand [144, 145], and up to $25 \mathrm{MA} \mathrm{m}^{-2}$ for $2600 \mathrm{~A} \mathrm{~mm}^{-2} \mathrm{Nb}_{3} \mathrm{Sn}$ strand [145] (when designed with a graded low-field/high-field winding pack) all at $4.2 \mathrm{~K}$. Further, high-temperature-superconductor (HTS) cables such as the twisted stacked-tape cable conductor CICC show promise for providing $40 \mathrm{MA} \mathrm{m}^{-2}$ or higher 

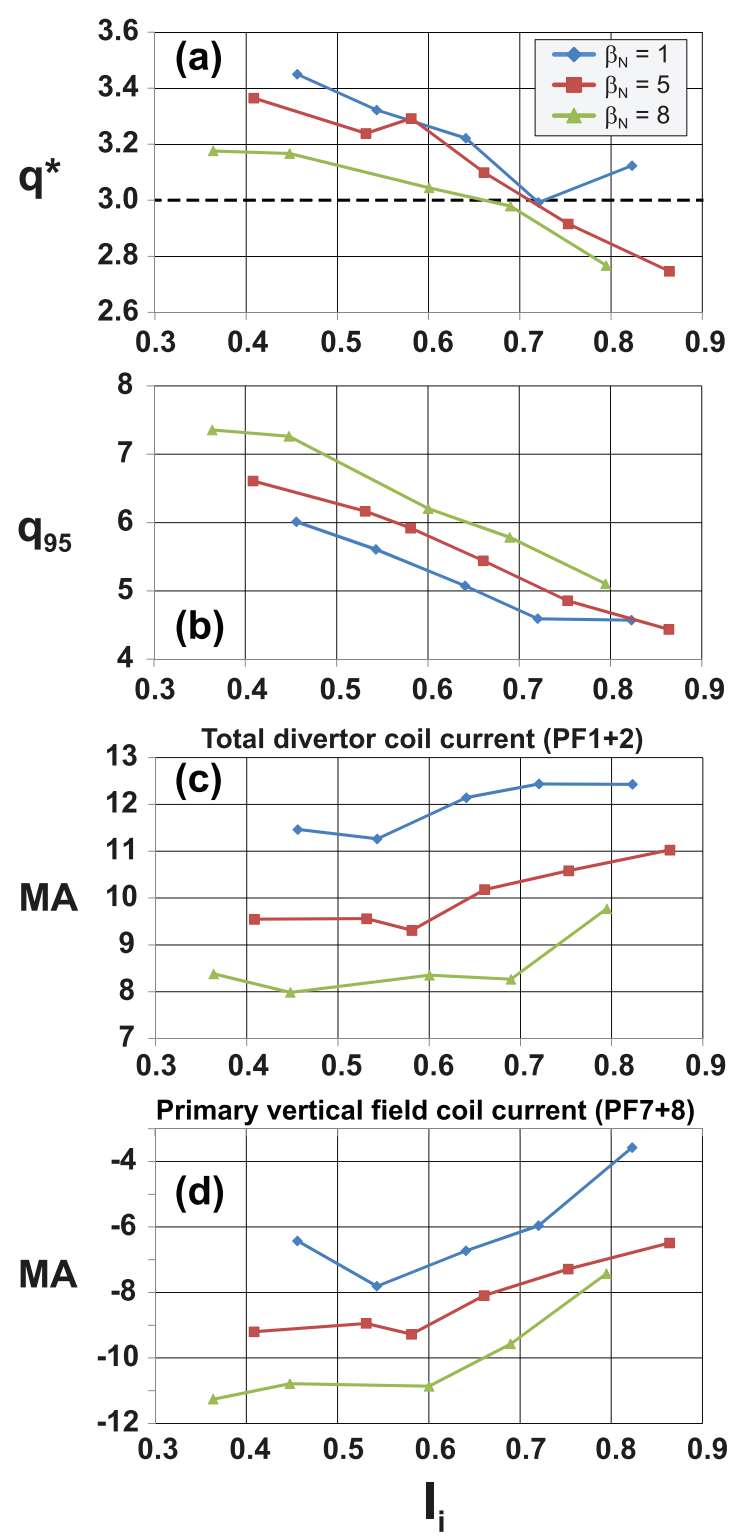

Figure 17. (a) Cylindrical safety factor $q^{*},(b)$ safety factor at 95\% flux surface $q_{95},(c)$ total inboard divertor coil current, and $(d)$ primary vertical field coil current for a range of $l_{\mathrm{i}}$ and $\beta_{\mathrm{N}}$ for $R_{0}=1.7 \mathrm{~m}$ ST-FNSF

winding-pack current density [146], and conductor on round core (CORC) cables have achieved up to $70 \mathrm{MA} \mathrm{m}^{-2}$ or higher winding-pack current density at fields up to $19 \mathrm{~T}$ in 'CORC4' samples in [147]. For the design study described here, 40 MA $\mathrm{m}^{-2}$ was targeted as the nominal current density value for HTS and also used to conservatively establish the design space for a radiation-resistant Bitter-plate coils.

Figure 18 shows the effective winding-pack current densities of the ST-FNSF PF coils with a $40 \mathrm{MA} \mathrm{m}^{-2}$ limit indicated by dashed lines. Figure 18( $a$ ) shows that PF1 has low current density ( $\leqslant 10 \mathrm{MA} \mathrm{m}^{-2}$ ) at the lowest $l_{\mathrm{i}}$ values studied, but reaches $30-40 \mathrm{MA} \mathrm{m}^{-2}$ at the highest $l_{\mathrm{i}}$ values. In contrast, figure $18(b)$ shows that $\mathrm{PF} 2$ requires approximately 30-50 MA m ${ }^{-2}$ for all cases treated, but lower current is required when PF1 is at its highest current. Thus, since the nuclear heating and damage to these divertor coils is too high for superconducting coils (see section 4.1), both PF1 and PF2 must use an advanced Bitter/helical plate approach. Figure 18(c) shows that a comparatively modest $\pm 7 \mathrm{MA} \mathrm{m}^{-2}$ is required for PF3 implying conventional $\mathrm{Cu}$ coil technology could be utilized, or possibly LTS or HTS if the thermal/neutron shielding is sufficient. Figure 18 $(d)$ shows that PF4 current is zero for most equilibrium scenarios and has non-zero current only for low $l_{\mathrm{i}} \leqslant 0.5$. Figure 18(e) shows that PF5 has a significant positive current/current density used to extend the divertor strike-point to larger major radius as shown in figure 14. Figure 18( $f$ ) shows that the PF6 current is small for low and intermediate $\beta_{\mathrm{N}}$ values for $l_{\mathrm{i}}=0.5-0.75$ but can have significant negative values for lower or higher $l_{\mathrm{i}}$. Figure $18(\mathrm{~g})$ shows that the PF7 current is highest in magnitude for the lowest $l_{\mathrm{i}}$ decreases to nearly zero current at the highest $l_{\mathrm{i}}$. In contrast, as shown in figure $18(\mathrm{~g})$, the PF8 current changes sign and varies from approximately $20 \mathrm{MA} \mathrm{m}^{-2}$ to $-20 \mathrm{MA} \mathrm{m}^{-2}$ as $l_{\mathrm{i}}$ is varied from the lowest to highest values. Coils PF4-7 all have current densities that exceed $20 \mathrm{MA} \mathrm{m}^{-2}$ for at least some equilibrium configurations and would therefore require either high critical-current-density-strand LTS or advanced HTS magnets.

\subsection{Divertor power handling}

As discussed in section 2.2.1, incorporating a divertor geometry capable of mitigating the projected high parallel heat flux of the divertor scrape-off-layer (SOL) is critical to the overall design of an ST-FNSF. Recent assessments of the divertor heat flux scaling in tokamaks [148, 149] finds an unfavorable scaling with plasma current in which the scrape-off-layer (SOL) heat flux width scales nearly inversely with plasma current. If realized, such SOL narrowing at high current projects to very narrow heat-flux channels and high peak heat flux values in next-step devices including ITER. The reference ST-FNSF scenario considered here has $R_{0}=1.7 \mathrm{~m}, P_{\text {fusion }}=160 \mathrm{MW}$, $P_{\mathrm{NBI}}=80 \mathrm{MW}$, and $Q_{\mathrm{DT}}=2$. Assuming $Z_{\mathrm{eff}}=2.0, \bar{Z}=1.25$, and $\bar{A}=1.6$ in the Goldston heuristic heat-flux model [149] and using other ST-FNSF parameters, the projected poloidalaverage $\lambda_{m}=1.8 \mathrm{~mm}$ with an outboard midplane value $\lambda_{m}^{*}=0.8 \mathrm{~mm}$ [148]. Taking $\lambda_{\text {sol }}=\lambda_{m}^{*}=\lambda_{\text {Goldston }}^{*}$ appears to be a reasonable estimate [150], but the power-spreading value (the parameter $S$ in the Eich model) appears to be more divertor geometry dependent and not amenable to scaling from simple divertor or main plasma parameters [150, 151].

In order to proceed, it is assumed that $w_{\mathrm{pvt}} \approx \lambda_{\mathrm{sol}} \approx \lambda_{\text {Goldston }}^{*}$ in the Makowski nomenclature [150] as may be appropriate for more closed divertor geometries and small $\lambda_{\mathrm{sol}} \approx 1 \mathrm{~mm}$ [151]. The integral heat flux width is then given approximately by [150] $\lambda_{\text {Eich-int }}=\lambda_{\text {sol }}+1.64 w_{\text {pvt }} \approx 2.1 \mathrm{~mm}$ and this width can be used to relate the total power flux in the SOL to the peak heat flux at the target. It is further assumed that $80 \%$ of the total power flux is radiated away either from the core or edge prior to reaching the divertor target plate, that the SOL heat flux is evenly split between upper and lower divertors, and that $80 \%$ of the total SOL heat flux is exhausted to the outboard divertors (see section 2.2.2). Figures $19(a)$ and $(b)$ show that 

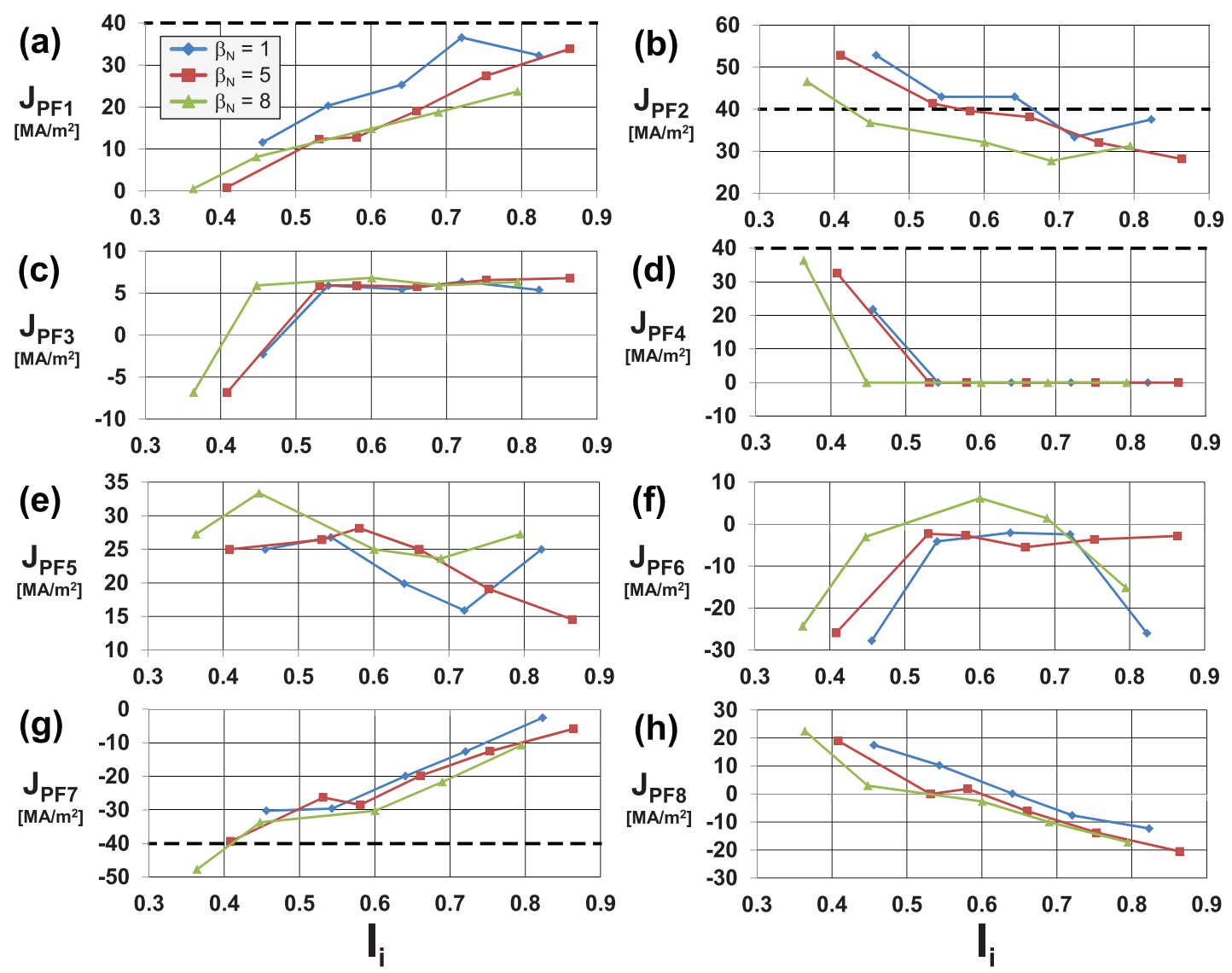

Figure 18. Poloidal field coil current densities $J_{\mathrm{PF}}$ for a range of $l_{\mathrm{i}}$ and $\beta_{\mathrm{N}}$ for $R_{0}=1.7 \mathrm{~m}$ ST-FNSF.
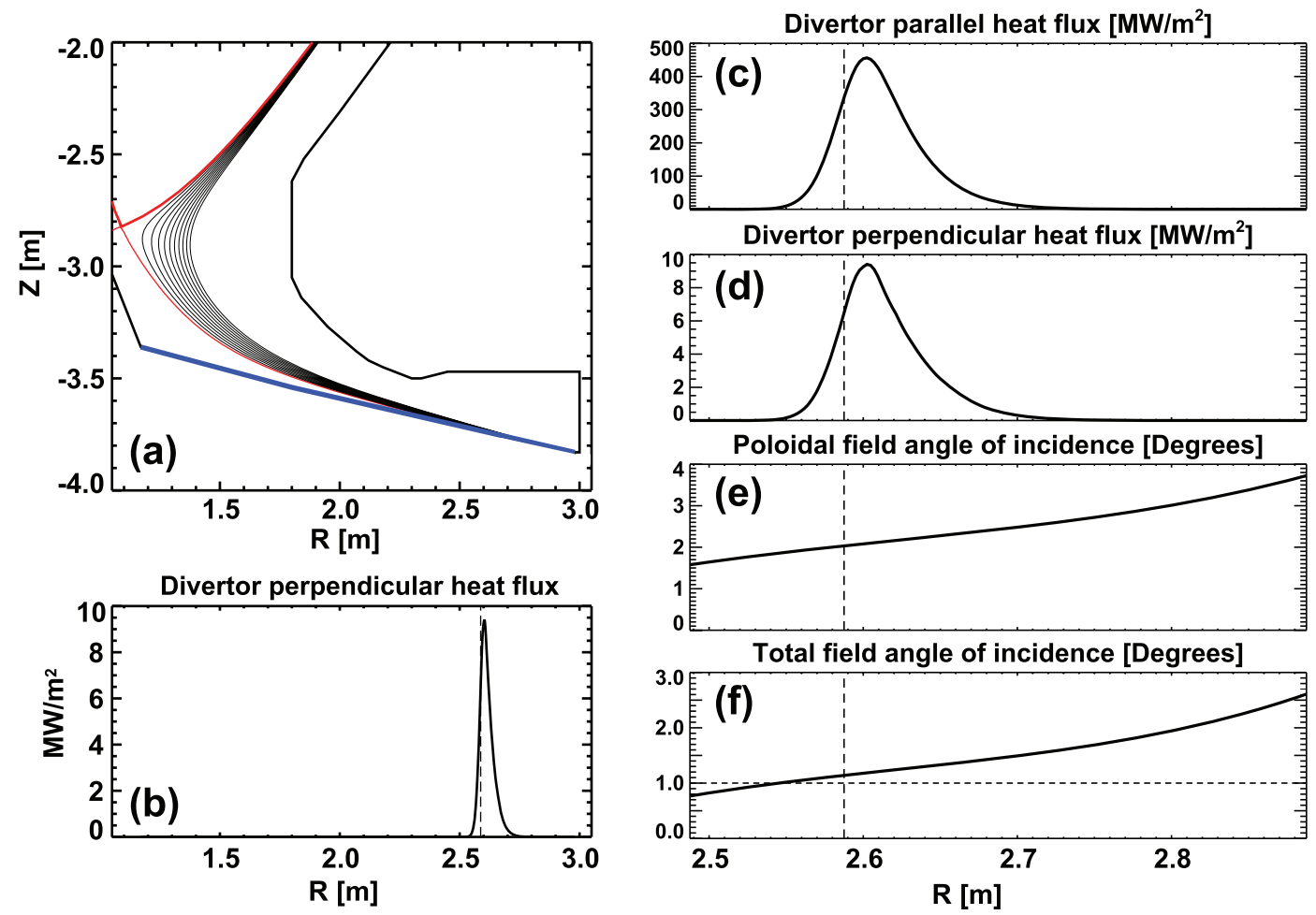

Figure 19. (a) Poloidal flux contours in the outboard horizontal divertor region with separatrix shown in red, target plate in blue, and limiter in black, and $(b)$ perpendicular heat flux profile at target plate. Radial profiles near strike-point radius (vertical dashed line) of: $(c)$ parallel heat flux, $(d)$ perpendicular heat flux, $(e)$ poloidal field angle of incidence, and $(f)$ total magnetic field angle of incidence. 

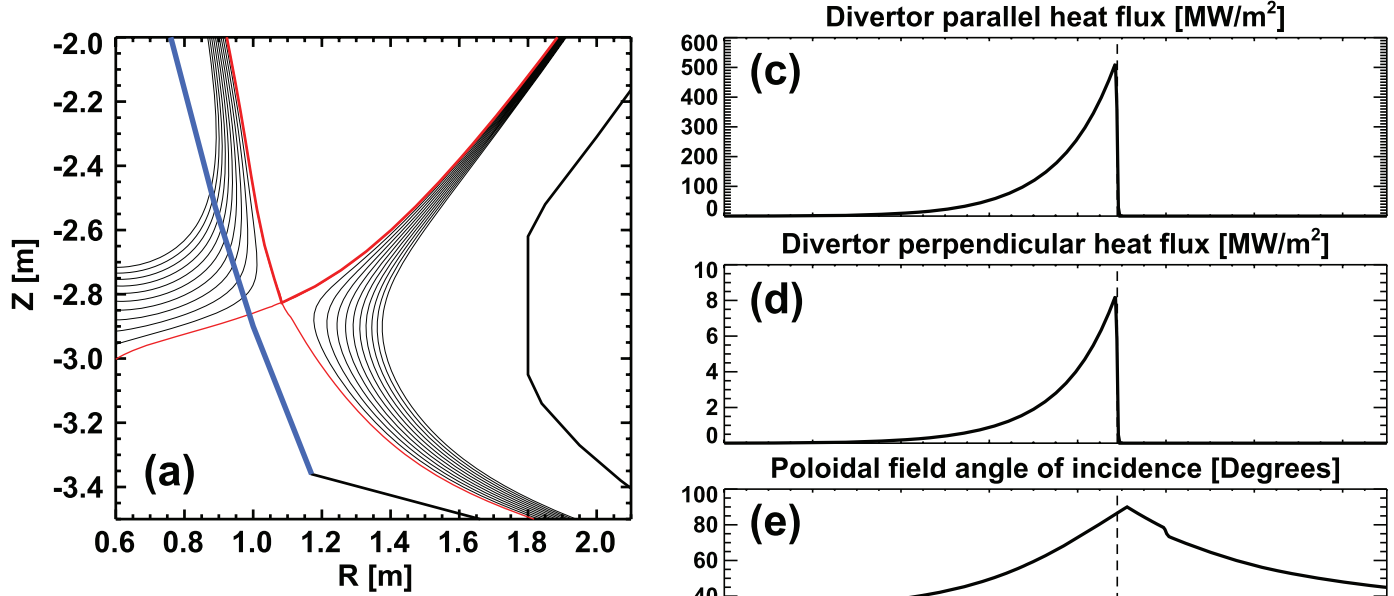

Divertor perpendicular heat flux $\left[\mathrm{MW} / \mathrm{m}^{2}\right]$

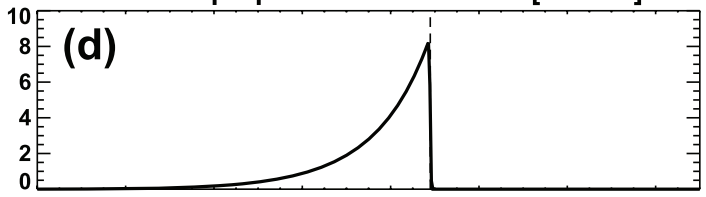

Poloidal field angle of incidence [Degrees]
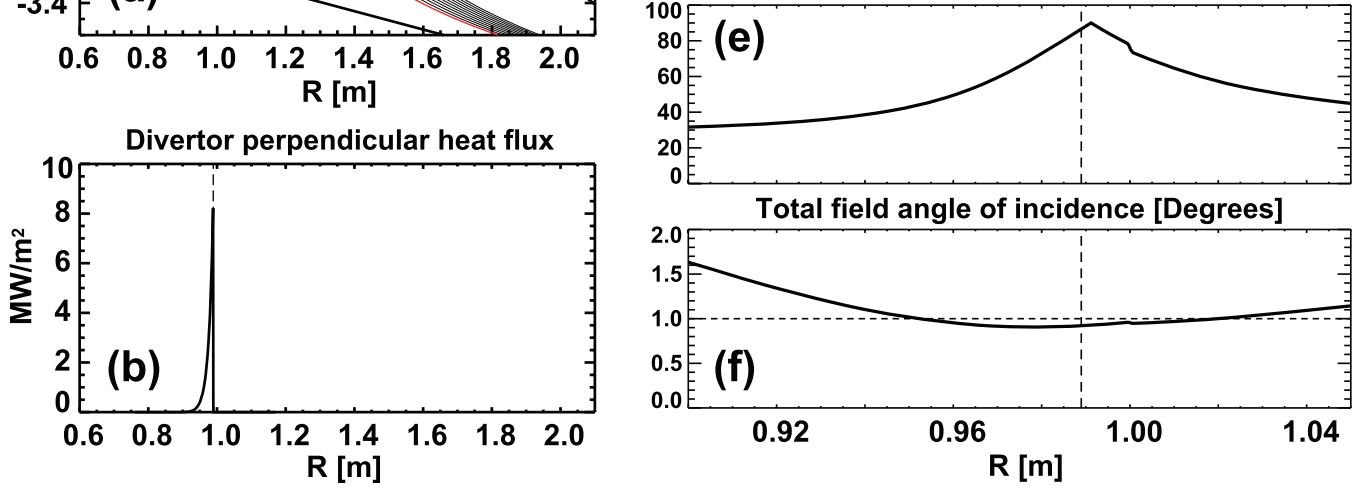

Figure 20. (a) Poloidal flux contours in the inboard vertical divertor region with separatrix shown in red, target plate in blue, and limiter in black, and $(b)$ perpendicular heat flux profile at target plate. Radial profiles near strike-point radius (vertical dashed line) of: $(c)$ parallel heat flux, $(d)$ perpendicular heat flux, $(e)$ poloidal field angle of incidence, and $(f)$ total magnetic field angle of incidence.

the peak divertor heat flux on the outboard divertor is below $10 \mathrm{MW} \mathrm{m}{ }^{2}$ with the above assumptions. Figures $19(c)$ and $(d)$ show a significant shift between the strike-point radius (vertical dashed line) and the location of peak heat flux due to the assumed power spreading into the private flux region along the long divertor leg. Without this spreading, a pure exponential heat flux profile would have a peak perpendicular heat flux of nearly $20 \mathrm{MW} \mathrm{m}^{-2}$. Figure $19(e)$ shows the poloidal field lines are nearly tangential to the target plate with an angle of incidence of $2^{\circ}$ at the divertor strike-point, and figure $19(f)$ shows the total magnetic field-line angle of incidence is $\approx 1^{\circ}$ at the strike-point.

Similarly, figures $20(a)$ and $(b)$ also show that the peak divertor heat flux on the inboard divertor is also below $10 \mathrm{MW} \mathrm{m}^{-2}$ with the above assumptions. However, figures 20(c) and (d) do not show a significant shift between the strike-point radius (vertical dashed line) and the location of peak heat flux because the assumed spreading width $w_{\text {pvt }} \approx \lambda_{\text {sol }}$ is much narrower than the highly poloidal-fluxexpanded strike-point region which is relatively close to the primary x-point. Figure 20(e) shows the poloidal field lines are nearly perpendicular to the target plate with an angle of incidence of $87^{\circ}$ at the divertor strike-point, and figure $20(f)$ shows the total magnetic field-line angle of incidence is again $\approx 1^{\circ}$ at the strike-point. It is important to note that conditions with peak heat flux $\leqslant 10 \mathrm{MW} \mathrm{m}^{-2}$ in both the outboard and inboard divertors are in principle obtainable without detachment, and detached conditions would further reduce the peak heat flux and reduce sputtering and erosion.

To more accurately examine the prospects for achieving heat fluxes $\leqslant 10 \mathrm{MW} \mathrm{m}^{-2}$ and temperatures of $2-10 \mathrm{eV}$ in the divertor region of ST-FNSF [123, 152], simulations of the SOL and divertor have been carried out using the SOLPS code [153]. This code uses a 2D fluid treatment of the plasma transport (using the B2 code [154]), coupled to a Monte-Carlo neutral transport calculation of the recycled neutrals (using EIRENE [155]). These calculations use similar assumptions as used in initial SOLPS calculations comparing different power exhaust geometries [123] and those assumptions are repeated here. In particular, transport is assumed to be classical parallel to the magnetic field (with kinetic corrections), and cross-field transport is governed by user-specified anomalous transport coefficients. Since a physics-based prediction of these coefficients is not readily available, they have instead been chosen to produce a SOL width that is in the range predicted by the multi-machine scaling experiments. The power flowing to the plasma edge is input as $50 \mathrm{MW}$ in the simulations; i.e. it is assumed that $55 \%$ of the $110 \mathrm{MW}$ total plasma heating power (80 MW NNBI, $30 \mathrm{MW}$ alpha heating) is dissipated by radiation from the core. The assumed edge power is still well above the L-H transition threshold [156], estimated to be 15-30 MW. The density at the core-most grid cell is set as a boundary condition and is used in the density scans to be described below. Radiation from nitrogen seeding at a fixed fraction of $2 \%$ is included.

Figure 21 shows SOLPS simulation results for two combinations [123] of particle diffusivity (D) and perpendicular thermal conductivity $(\chi)$ with a fixed ratio of $D / \chi=0.3 \mathrm{~m}^{2} \mathrm{~s}^{-1}$ / $1 \mathrm{~m}^{2} \mathrm{~s}^{-1}$ (blue) and $0.075 \mathrm{~m}^{2} \mathrm{~s}^{-1} / 0.25 \mathrm{~m}^{2} \mathrm{~s}^{-1}$ (red) to scan a range of heat-flux widths consistent with existing models and heuristic estimates. Figure 21 shows several exhaust parameters as a function of electron density at the outboard 


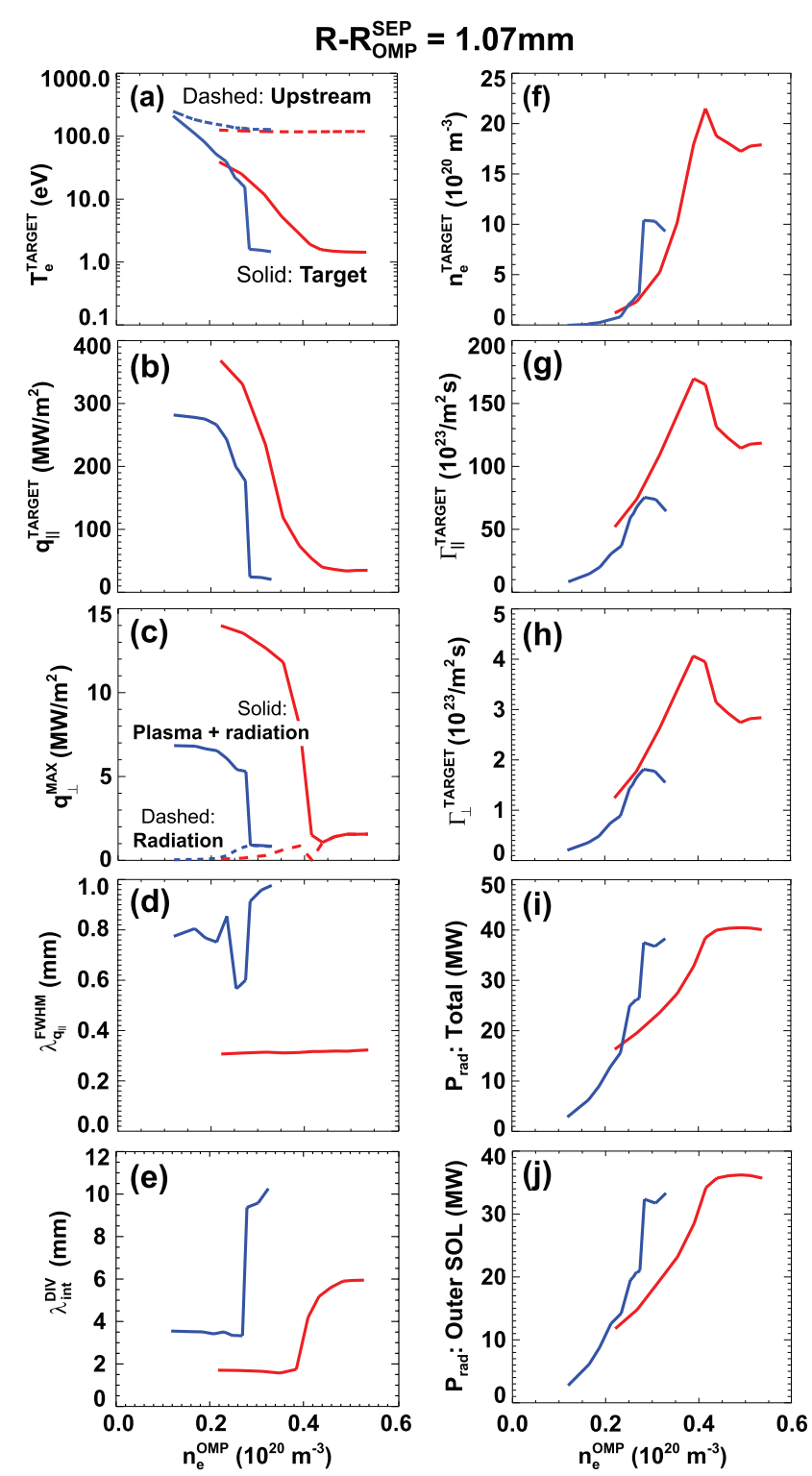

Figure 21. Results from SOLPS divertor power exhaust calculations for two different combinations of $D / \chi=0.3 / 1.0 \mathrm{~m}^{2} \mathrm{~s}^{-1}$ (blue) and $0.075 / 0.25 \mathrm{~m}^{2} \mathrm{~s}^{-1}$ (red).

mid-plane (OMP) $\left(n_{\mathrm{e}}^{\mathrm{OMP}}\right)$ including: (a) electron temperature at divertor target, (b) parallel heat flux at divertor target, (c) peak perpendicular heat flux at target, (d) full-width half-maximum parallel heat-flux width, (e) integral heat flux width at divertor target, (f) electron density at target, (g) parallel particle flux at target, (h) perpendicular particle flux at target, (i) total radiated power in divertor, and $(\mathrm{j})$ radiated power in outer scrapeoff-layer (SOL). Unless otherwise noted, these parameters are computed on flux-tubes $\approx 1.07 \mathrm{~mm}$ into the $\mathrm{SOL}$ as measured at the outboard midplane. As is evident from figures 21(a) $-(c)$, substantial reductions in divertor target plasma temperature and parallel and perpendicular heat fluxes occur in the range of $n_{\mathrm{e}}^{\mathrm{OMP}}=0.25-0.4 \times 10^{20} \mathrm{~m}^{-3}$ for the range of diffusion coefficients treated. As shown in figure 21(e), the computed integral heat flux width $\approx 1.5 \mathrm{~mm}$ for the $D / \chi=0.075 \mathrm{~m}^{2} \mathrm{~s}^{-1} /$ $0.25 \mathrm{~m}^{2} \mathrm{~s}^{-1}$ case (red) is somewhat below the empirically estimated value of $\approx 2.1 \mathrm{~mm}$ indicating that this narrower
SOL width case may provide an upper bound on peak heat fluxes that might be expected. For this narrowest SOL case there is potentially a relatively narrow density operating window $n_{\mathrm{e}}^{\text {OMP }}=0.35-0.410^{20} \mathrm{~m}^{-3}$ that simultaneously satisfies $q_{\perp}^{\mathrm{MAX}} \leqslant 10 \mathrm{MW} \mathrm{m}{ }^{-2}$ and $1-2 \mathrm{eV} \leqslant T_{\mathrm{e}}^{\mathrm{TARGET}} \leqslant 10 \mathrm{eV}$, i.e. a temperature range that avoids complete detachment and large material sputtering. As shown in figures $21(f)-(h)$, the target density and particle fluxes rise secularly as the edge density is increased until the onset of complete detachment $\left(n_{\mathrm{e}}^{\mathrm{OMP}} \geqslant 0.4\right.$ $-0.45 \times 10^{20} \mathrm{~m}^{-3}$ ) after which these parameters decrease while the radiated powers remain roughly constant as shown in figures $21(i)-(j)$. If the SOL heat-flux width is wider than obtained in this most pessimistic calculation, then the den-

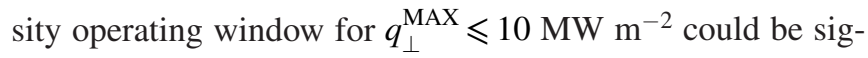
nificantly wider as shown by the blue curves in figure $21(c)$, although $T_{\mathrm{e}}^{\mathrm{TARGET}}$ would exceed $10 \mathrm{eV}$.

If a wider heat-flux width is not accessible, increasing the edge radiation fraction is an option, but could degrade core energy confinement [125]. Increasing the core radiation fraction is another potential option to increase the divertor density operating window, but thermal stability of the core plasma would need to be assessed [125], though it should be noted that thermal stability should be improved by the modest $Q \approx 2$ of the ST-FNSF operating point considered here. It should also be noted that there is significant flexibility in the radiating impurity and electron density operating point since the nominal line-average density of $3 \times 10^{20} \mathrm{~m}^{-3}$ (at $f_{\text {Greenwald }}=0.8$ ) exceeds the above identified OMP density for detachment control by a factor of 7-8, and provides a factor of two in density reduction flexibility even if the OMP density is only $25 \%$ of the line-average. Finally, more recent UEDGE calculations including an up-stream cryo-pumping duct and baffling for long-leg divertors like those shown here indicate the detachment front may remain stable even under fully detached conditions [152], and this could allow operation over a relatively wide density operating range while remaining fully detached. Issues such as these are planned to be studied in upcoming MAST-U divertor experiments [12] and could also be studied at conventional aspect ratio and high-field in the proposed ADX experiment [157].

\subsection{Plasma sustainment and ramp-up}

Neutral beam injection has been identified as well-suited to providing heating and current drive for ST-FNSF as described in section 2.1.5. However, while neutral beams have positive attributes including high current drive efficiency, no accessibility/density limit, and no plasma facing components near the plasma boundary, neutral beams do require substantial testcell floor space and can require large apertures and penetrations in the first-wall and blanket which can adversely impact neutron shielding and reduce tritium breeding. There are also practical limits on maximum tangency radius of injection due to space constraints between the toroidal field coil outer legs and due to maximum toroidal field ripple constraints. Further, whatever NBI configuration is chosen for the highperformance phases of ST-FNSF operation, the same systems 

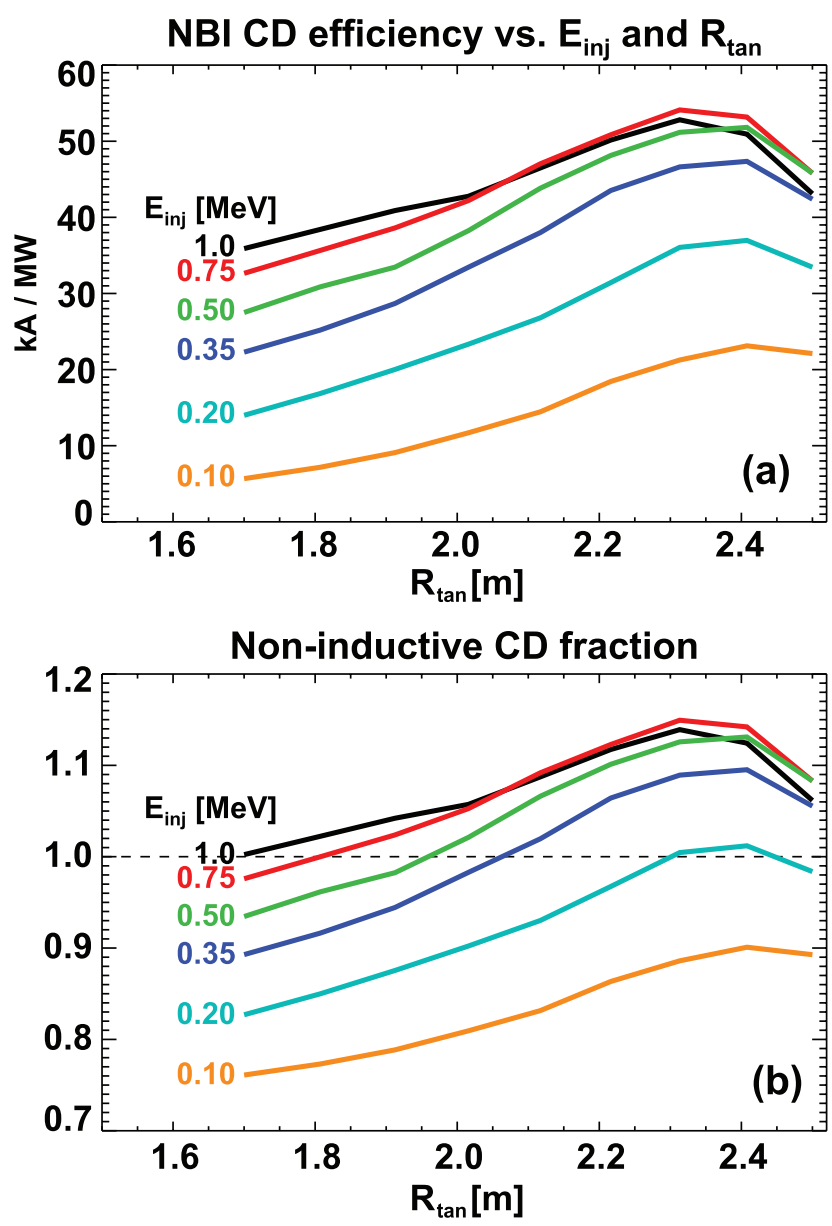

Figure 22. (a) Neutral beam current drive efficiency versus tangency radius of injection $R_{\tan }$ and injection energy $E_{\mathrm{inj}}$ for a D-D target plasma, and $(b)$ non-inductive current drive fraction for a $I_{\mathrm{P}}=7.5 \mathrm{MA}$ target plasma heated with $60 \mathrm{MW}$ of NNBI.

must also be compatible with providing NBI plus bootstrap current overdrive since ST-FNSF will have no or only a small ohmic solenoid. For all these reasons, careful consideration must be given to the injection geometry of the NBI systems to make the NBI compatible with the overall physics, engineering, and neutronics constraints.

2.4.1. Neutral beam current drive optimization. An important constraint on the overall device design and in particular heating and current drive system is that sufficiently high plasma current can be sustained to confine D-T fusion alpha particles even in the absence of alpha heating. With this capability it is in principle possible to continuously vary the fusion power by varying the mix of $\mathrm{D}$ and $\mathrm{T}$ fuel from pure $\mathrm{D}-\mathrm{D}$ to $50-50 \%$ D-T. Figure 22 shows how negative neutral beam injection (NNBI) current drive efficiency (in $\mathrm{kA} \mathrm{MW}^{-1}$ ) scales with injection energy and tangency radius of injection as calculated using the free-boundary TRANSP code [158, 159] and NUBEAM [13-15] module. The target plasma is fixed for this scan and has $A=1.8, R_{0}=1.7 \mathrm{~m}, B_{\mathrm{T}}=2.9 \mathrm{~T}, I_{\mathrm{P}}=7.5 \mathrm{MA}$, $f_{\mathrm{GW}}=0.7, q_{\min }=2.2, q_{95}=8.9$, and $\beta_{\mathrm{N}}=4.1$ consistent with $H_{98}=1.2$ if $80 \mathrm{MW}$ of DD NNBI was injected and absorbed. Fixed temperature and density profiles are used (scaled from NSTX profiles without constraints on the thermal or particle diffusivities) in order to provide fixed plasma target parameters for computing NBI current drive profiles and efficiency scalings for conditions similar to the fully noninductive DD targets shown in figure 23. In particular, the target plasma has $T_{\mathrm{e}}(0)=8.6 \mathrm{keV}, T_{\mathrm{i}}(0)=11.6 \mathrm{keV}$, and the $Z_{\text {eff }}$ profile is held fixed in all scans and has a value of 2 from the magnetic axis to approximately $r / a=0.7$ and increases to 2.5 at the plasma boundary. As shown in figure 22(a), the current drive efficiency increases rapidly with injection energy from $0.1-0.35 \mathrm{MeV}$, then increases more slowly up to 0.5 $\mathrm{MeV}$, and above $0.5 \mathrm{MeV}$ increases little or begins to decrease slightly at large $R_{\tan }$ due to shine-through. The maximum CD efficiency is achieved for $R_{\mathrm{tan}}=2.3-2.4 \mathrm{~m}$ for a $R_{0}=1.7 \mathrm{~m}$ ST-FNSF device. Thus, the optimal injection energy is apparently $0.5-0.75 \mathrm{MeV}$, and the optimal radius range is approximately $R_{\tan }=1.7-2.4 \mathrm{~m}$ for control of $J(r)$ and $q(0) / q_{\min }$ while avoiding excessive shine-through at larger $R_{\tan }$. However, the lower $E_{\mathrm{NBI}}$ value $=0.5 \mathrm{MeV}$ in this optimal range is chosen to reduce fast-ion losses at lower $I_{\mathrm{P}}$ values during the current ramp-up as discussed in section 2.4.3. Figure 22(b) shows the non-inductive $\mathrm{CD}$ fraction that would be achieved for a $I_{\mathrm{P}}=7.5 \mathrm{MA} \mathrm{D}-\mathrm{D}$ target plasma heated with $60 \mathrm{MW}$ of NNBI and shows that fully non-inductive operation at this plasma current would require some power to be injected with $R_{\mathrm{tan}}>2$ $\mathrm{m}$ if $E_{\mathrm{NBI}}<0.5 \mathrm{MeV}$. These calculations also enable optimization of the power versus tangency radius of injection in order to provide a desired total current density profile with $q_{\text {min }}>2$ without generating deep reversed shear. Such a power versus tangency radius optimization is utilized in the next section to study fully-non-inductive equilibrium profile dependence on confinement and density.

2.4.2. Steady-state scenario dependence on density and confinement. To more systematically and self-consistently investigate the plasma performance as a function of confinement and density, free-boundary TRANSP and NUBEAM are again used taking scaled electron density and temperature profiles from NSTX but this time solving for the ion temperature profile assuming neoclassical ion thermal transport across the entire profile based on NSTX results where $\chi_{\mathrm{i}} \approx \chi_{\mathrm{i}-\text { neoclassical }}$ was observed over a majority of the plasma outer minor radius in rapidly-rotating NBI-heated H-mode plasmas [68, 69]. The NNBI has injection energy $E_{\text {inj }}=0.5 \mathrm{MeV}$ and the injection geometry and source powers are fixed with values $R_{\text {tan }}=170 / 201 / 231 / 240 \mathrm{~cm}$ and $P_{\mathrm{NBI}}=5 / 25 / 25 / 25 \mathrm{MW}$ for 80 MW total injected power. The confinement multiplier $H_{98}$ and normalized density $f_{\mathrm{GW}}$ are varied to assess scenario performance. D-D plasmas with $R_{0}=1.7 \mathrm{~m}$ and $B_{\mathrm{T}}=2.9 \mathrm{~T}$ are assessed first to establish a performance baseline in the absence of alpha heating.

As shown in figure 23(a) for D-D plasmas, the total $\beta_{\mathrm{N}}$ is primarily a function of $H_{98}$ and $\beta_{\mathrm{N}} \leqslant 6$ for all density and confinement values used. Figure $23(b)$ shows that the thermal fraction of the total $\beta_{\mathrm{N}}$ increases if either $H_{98}$ or $f_{\mathrm{GW}}$ are increased. Figure 23(c) shows that the plasma current is maximized by increasing confinement or decreasing density, and the achievable plasma current in D-D is $~ 10 \mathrm{MA}$ at $H_{98}=1.5$ and $f_{\mathrm{GW}}=0.5$. Figure $23(d)$ shows the beam 
(a)

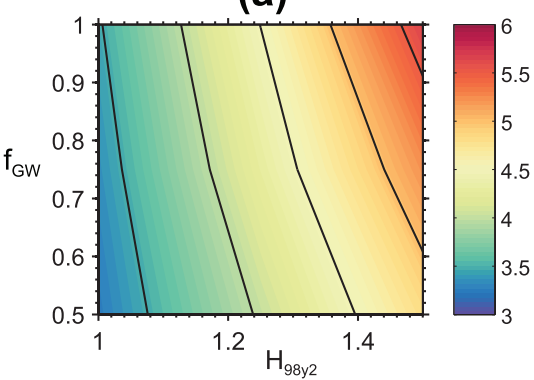

(c)

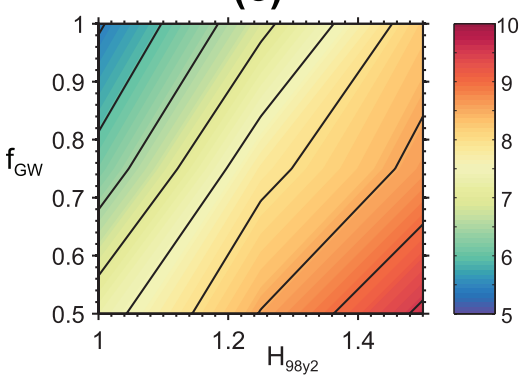

(e)

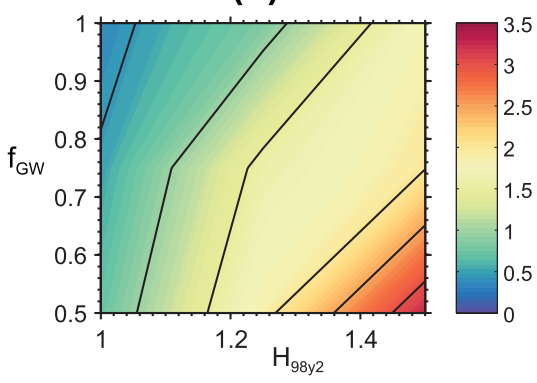

(b)

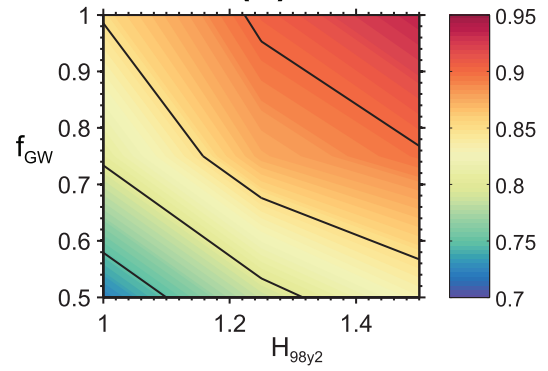

(d)

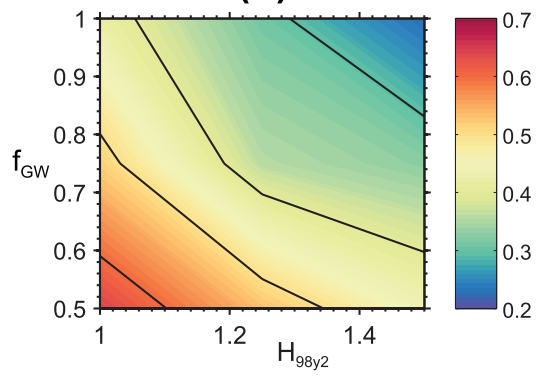

(f)

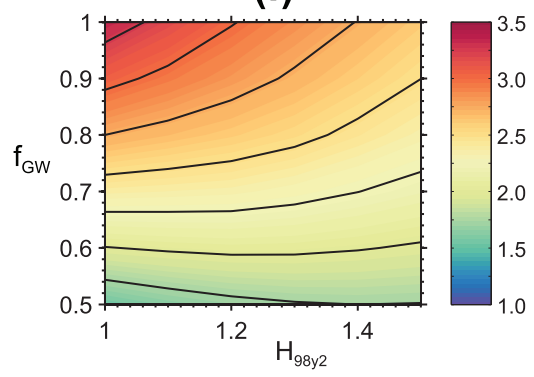

Figure 23. Plasma performance parameters computed by TRANSP as a function of confinement multiplier $H_{98 y_{2}}$ and normalized density $f_{\mathrm{GW}}$ for D-D plasma. (a) Total $\beta_{\mathrm{N}}$. (b) Thermal $\beta_{\mathrm{N}}$ fraction. (c) Plasma current (MA). (d) Beam current drive fraction. (e) Fusion power (MW). $(f) q_{\min }$.

current drive fraction is minimized to $20-25 \%$ for the highest density and confinement multipliers considered. Figure 23(e) shows the expected small fusion power for these D-D cases, and figure $23(f)$ shows that $q_{\text {min }}$ remains above 1 for all confinement and density cases considered, and maintaining $q_{\text {min }} \geqslant 2$ requires maintaining $f_{\mathrm{GW}}>0.6$.

Otherwise identical confinement and density scans have been performed assuming 50-50\% D-T main ion composition as shown in figure 24. As shown in figure 24(a) the total $\beta_{\mathrm{N}}$ is again primarily a function of $H_{98}$, but due to the additional alpha heating, $\beta_{\mathrm{N}}$ values up to $8-9$ are accessible for $H_{98}=1.5$, so either confinement or tritium fraction control is needed to avoid $\beta_{\mathrm{N}}$ limits. Figure $24(b)$ shows that the thermal fraction of the total $\beta_{\mathrm{N}}$ increases if either $H_{98}$ or $f_{\mathrm{GW}}$ are increased with trends similar to that observed for the D-D case. Figure 24(c) shows that at higher confinement multipliers the plasma current depends primarily on $\mathrm{H}_{98}$, and the maximum achievable plasma current would be 11-12 MA at the highest $H_{98}$ values (which are likely MHD unstable). Figure $24(d)$ shows the beam current drive fraction is 5-10\% lower than for the D-D cases due to the additional alpha heating, increased thermal pressure, and increased bootstrap current. Figure 24(e) shows the expected strong dependence of fusion power on confinement multipliers with very high fusion powers theoretically possible at higher $H_{98}$. To limit $\beta_{\mathrm{N}} \leqslant 6$ likely requires either limiting $H_{98} \leqslant 1.35$ or decreasing the NNBI power and this would limit the maximum fusion power to approximately $300 \mathrm{MW}$. Figure $24(f)$ shows that $q_{\text {min }}$ again remains above 1 for all confinement and density cases considered, but $q_{\text {min }}$ decreases more rapidly with increasing high $H_{98}$ than observed for D-D targets.

Figure 25 shows TRANSP calculations of various profiles of a representative FNSF scenario. For this scenario, $A=1.8$, $R_{0}=1.7 \mathrm{~m}, H_{98}=1.3, f_{\mathrm{GW}}=0.7, I_{\mathrm{P}}=8.9 \mathrm{MA}, B_{\mathrm{T}}=2.9 \mathrm{~T}$, $f_{\mathrm{NICD}}=100 \%, f_{\mathrm{BS}}=65 \%, P_{\mathrm{NBI}}=80 \mathrm{MW}, E_{\mathrm{NBI}}=0.5 \mathrm{MeV}$, $P_{\text {fusion }}=200 \mathrm{MW}, Q_{\mathrm{DT}}=2.5, \beta_{\mathrm{N}}=5.5, W_{\mathrm{tot}}=58 \mathrm{MJ}$, and $W_{\text {fast }} / W_{\text {total }}=14 \%$. The alpha loss power is dominated by bad orbit loss which is $2.6 \%$ of the total heating power. Figure $25(a)$ shows that the ion temperature exceeds the electron temperature in the plasma core for the assumed neoclassical thermal ion diffusivity, and this is representative of hot-ion H-mode operation. Figure 25(b) shows fully non-inductive operation with the bootstrap current density peaking at mid-radius and in the pedestal region, and the NBI current density peaking on axis and at mid-radius. As shown in figure 25(c), the assumed electron density profile is centrally peaked, and like the 
(a)

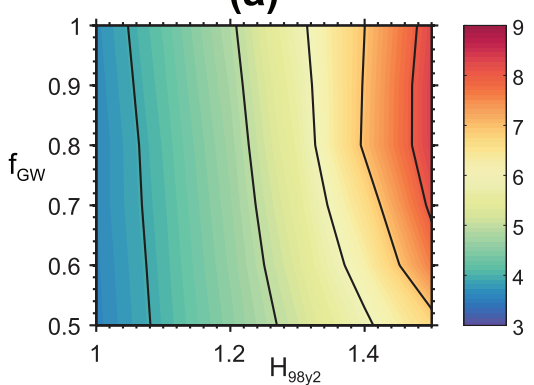

(c)

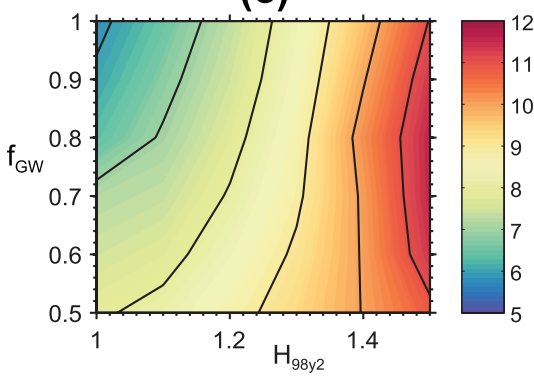

(e)

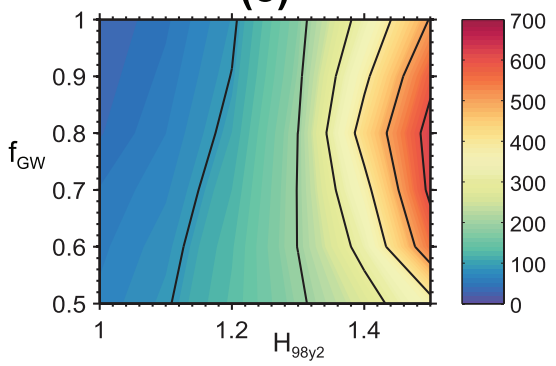

(b)

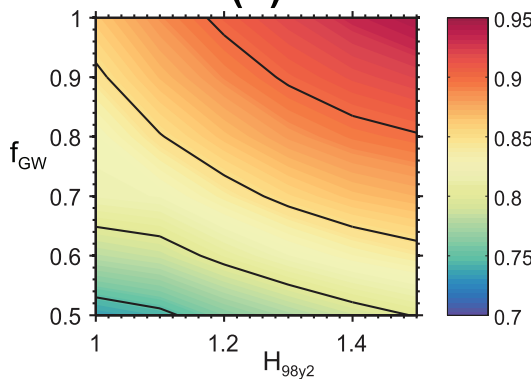

(d)

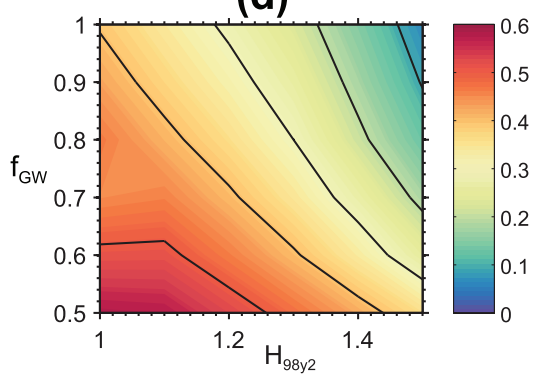

(f)

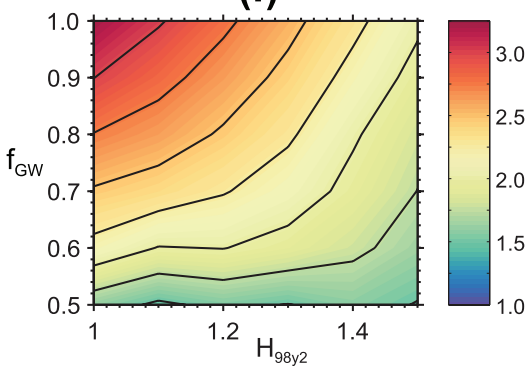

Figure 24. Plasma performance parameters computed by TRANSP as a function of confinement multiplier $H_{98 y 2}$ and normalized density $f_{\mathrm{GW}}$ for $50-50 \%$ D-T plasma. (a) Total $\beta_{\mathrm{N}}$. (b) Thermal $\beta_{\mathrm{N}}$ fraction. (c) Plasma current (MA). (d) Beam current drive fraction. (e) Fusion power (MW). ( $f$ ) $q_{\text {min }}$.

electron temperature profile is scaled from NSTX results and is not computed from first-principles transport calculations. As shown in figure $25(d)$, the safety factor profile has $q_{\min }>2$ and $q(0)=3.5$, and additional variations of injection radius, density, and confinement can be used to modify the minimum $q$ and $q$-shear in the core region (not shown).

As is the case for vertical stability (see section 2.1.2), the location of the conducting wall is very important for low-n kink stability when operating above the no-wall limit. Figure 26 shows low-n ideal kink stability analysis starting from the profiles shown in figure 25 . In this analysis, small near-axis current drive is added to decrease $q(0)$ to 2.6 (with $q_{\text {min }}$ remaining near 2.1$)$ to stabilize core interchange modes so only external kink modes are unstable. Figure 26(a) shows the limiter boundary (gray), plasma boundary (red), and set of outboard wall locations $\Delta R_{\text {wall }}$ scanned (blue) with conducting wall radial shift relative to the nominal first-wall location varying from 0 to $100 \mathrm{~cm}$. Figure $26(b)$ shows that the $n=1$ mode is the most stable with marginal wall location $0.9-1 \mathrm{~m}$ behind the first wall. Toroidal mode numbers $n=2$ and $n=3$ require a closer fitting wall for stabilization than $n=1$, and the exact marginal wall locations are sensitive to details of the pressure and current density profiles. Higher-n modes $n=4$ and 5 are found to be stable. These results show that $n=1$ wall stabilization can likely be supported by shells $50 \mathrm{~cm}$ behind the first wall as shown in figure 38 . However, wall stabilization of $n=2$ and 3 modes requires a closer fitting wall/shell as is required for stabilizing the $n=0$ mode for high $l_{\text {i }}$ plasmas at high elongation.

2.4.3. Plasma current ramp-up. Since ST-FNSF devices will have either no central solenoid or only a very small start-up flux, plasma current ramp-up via current overdrive is a critical issue. Non-inductive current ramp-up is envisioned to be achieved by non-inductive overdrive ( $>100 \%$ non-inductive current drive) using the same current drive systems used for sustainment, i.e. neoclassical bootstrap current and NBI current drive. To study the achievable range of plasma currents that can be supported using NNBI + bootstrap current, systematic scans of beam tangency radius combination, turn-on sequence, source power, and voltage were performed using the same simulation methodology used in section 2.4 .2 but with fixed confinement multiplier $H_{98}=1.15$ in the range of experimentally achieved values, fixed normalized density at a relatively high $f_{\mathrm{GW}}=0.95$ to maximize beam absorption at low plasma current, and assumed D-D operation with no 

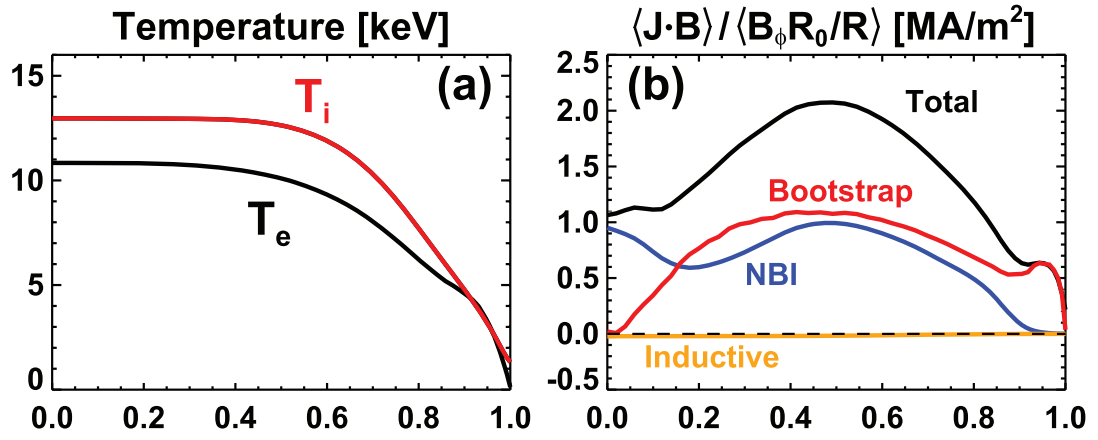

Density $\left[10^{20} \mathrm{~m}^{-3}\right]$

Safety factor
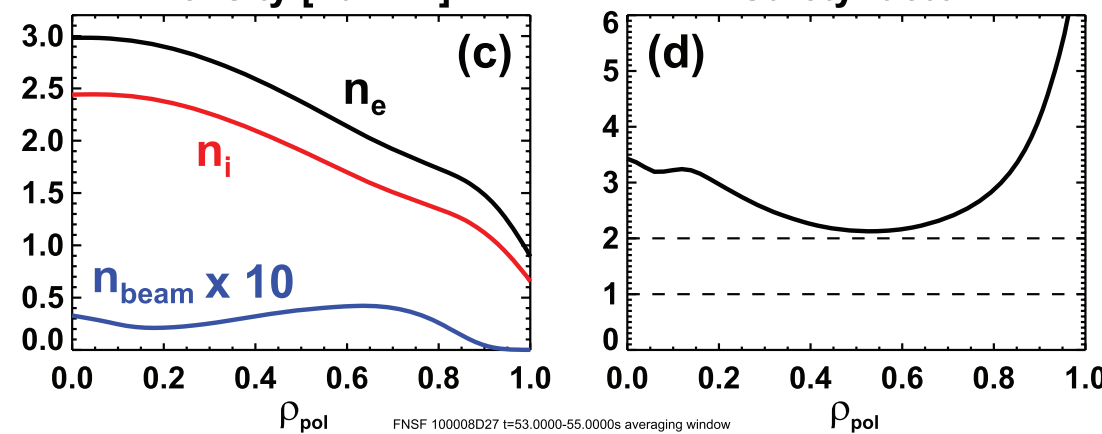

Figure 25. Profiles from a TRANSP simulation of a D-T ST-FNSF plasma scenario with $200 \mathrm{MW}$ of fusion power.
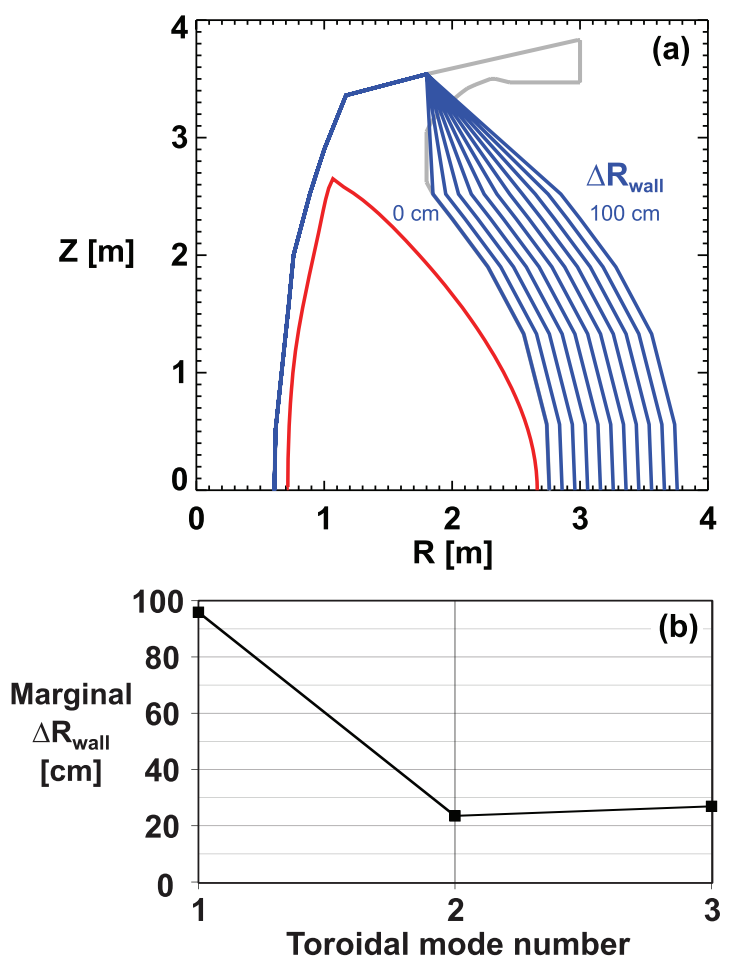

Figure 26. Low-n kink stability analysis for the plasma in figure 25 showing $(a)$ limiter boundary (gray), plasma boundary (red), and wall positions tested (blue), and (b) kink marginal wall position versus toroidal mode number.

additional alpha heating power. An important goal of these scans is to identify scenarios with low fast-ion losses, either monotonic or only weakly-reversed $q$ shear over a wide range of total power and plasma current, and a smooth trajectory of all global parameters of interest from low to high current $I_{\mathrm{P}}=2-8 \mathrm{MA}$. Preference is given to scenarios that only utilize

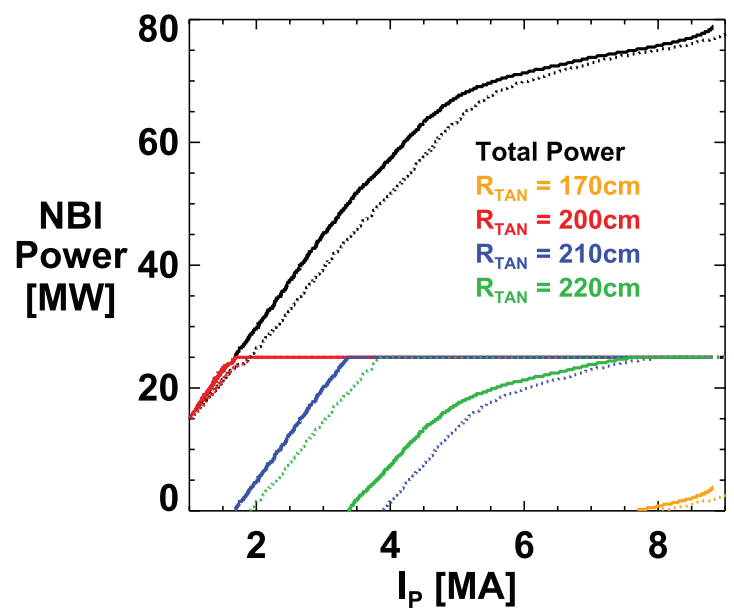

Figure 27. Total and individual source NNBI heating powers versus plasma current for $R_{\tan }=170 / 200 / 210 / 220 \mathrm{~cm}$ injection tangency radii for two different beam voltage combinations: 0.5/0.1/0.5/0.5 $\mathrm{MeV}$ (solid) and $0.5 \mathrm{MeV}$ for all $R_{\mathrm{TAN}}$ (dashed).

a fixed subset of the available beam tangency radii, can utilize a simple sequential beam turn-on, and that use the ST-FNSF baseline NNBI beam energy of $E_{\mathrm{NBI}}=0.5 \mathrm{MeV}$.

Two favorable NBI scenarios have been identified from these scans, and figure 27 plots the total (non-inductive) plasma current versus total and individual source NBI heating power for these two cases both with fixed TF coil current corresponding to $B_{\mathrm{T}}=2.9 \mathrm{~T}$ at $R_{0}=1.7 \mathrm{~m}$. As seen in the figure, the $R_{\mathrm{TAN}}=200 \mathrm{~cm}$ source is used first at low $I_{\mathrm{P}}$, followed by either the 210 or $220 \mathrm{~cm}$ source, and finally the $170 \mathrm{~cm}$ source is used at the highest current values at lower power for central $q$ control. The two optimized cases have the same $R_{\mathrm{TAN}}=170 / 200 / 210 / 220 \mathrm{~cm}$ injection tangency radii but two different beam voltage combinations: $0.5 / 0.1 / 0.5 / 0.5 \mathrm{MeV}$ 

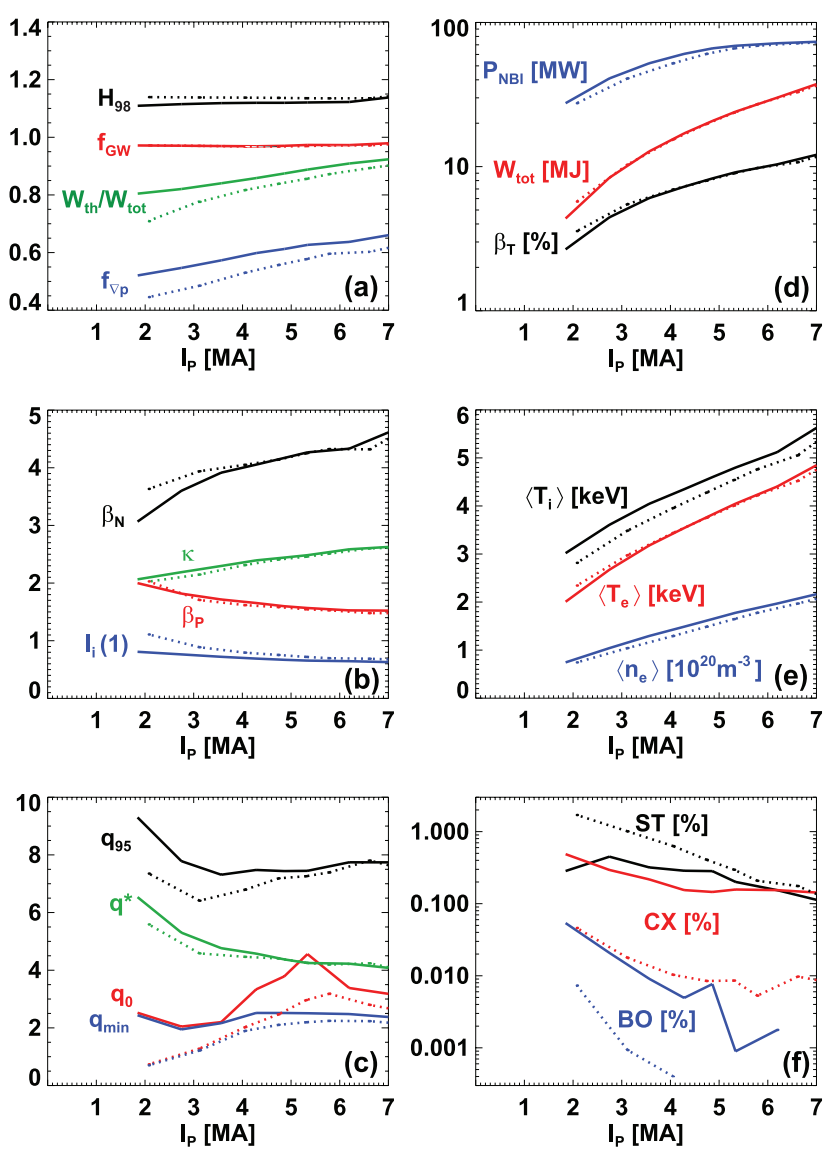

Figure 28. Plasma performance parameters computed by TRANSP a as a function of plasma currrent for $100 \%$ non-inductive D-D plasmas.

(solid) and $0.5 \mathrm{MeV}$ for all $R_{\mathrm{TAN}}$ (dashed), and the variation in $200 \mathrm{~cm}$ NBI energy is used to bracket the effect of beam energy on central $q$ value (and other parameters) at low plasma current. In all of these scans, an artificially high plasma resistivity is used to accelerate TRANSP calculation of equilibrated fully-noninductive current profiles to approximate a nearly-equilibrated slow ramp-up that might be used in an actual ST-FNSF ramp-up.

Figure 28 shows the global parameter variations versus current for the power trajectories shown in figure 27. Figure 28(a) shows the fixed $H_{98}$ and $f_{\mathrm{GW}}$ and shows the thermal stored energy fraction $W_{\text {th }} / W_{\text {tot }}$ increases from $70-80 \%$ at $I_{\mathrm{P}} \approx 2 \mathrm{MA}$ to $90 \%$ at full current, and the fraction of pressure-gradientdriven (bootstrap + diamagnetic) current $f_{\nabla p}$ increases from $45-50 \%$ to $60-65 \%$. Figure $28(b)$ shows $\beta_{\mathrm{N}}$ increases from $3-3.5$ to 4.5 at full current, while the elongation $\kappa$ increases from 2 to 2.6 as the internal inductance $l_{\mathrm{i}}$ decreases from $0.8-0.9$ to 0.6 from low $\left(I_{\mathrm{P}}=2 \mathrm{MA}\right)$ to high $\left(I_{\mathrm{P}}=7 \mathrm{MA}\right)$ current. Figure $28(c)$ shows $q_{95} \approx 7-9$ at low current and has a similar value $q_{95} \approx 8$ at high current $I_{\mathrm{P}}=7 \mathrm{MA}$, while the cylindrical kink safety factor $q^{*}$ decreases from 6 to 4 from low to high current. The $q_{\mathrm{min}}$ and $q_{0}$ curves show that the $q$ profile is monotonic at lower current, but weakly reversed for $I_{\mathrm{P}}$ above $4 \mathrm{MA}$. These curves also show that the lower $E_{\mathrm{NBI}}=0.1 \mathrm{MeV}$ of the $R_{\mathrm{TAN}}=200 \mathrm{~cm}$ source (solid) helps maintain $q_{\text {min }}$ above 2 for all ramp-up currents. In contrast, higher $E_{\mathrm{NBI}}=0.5 \mathrm{MeV}$ for this source can drive too much central current and lower $q_{\min }$ to 1 at lower $I_{\mathrm{P}}=2 \mathrm{MA}$. This comparison shows the increased sensitivity of the $q$ profile to beam energy and injection radius at lower current values, and illustrates that more than one injection radius may be needed at low power to maintain $q_{\min }>2$ if elevated $q$ is necessary for MHD stability.

Figure 28(d) shows the plasma stored energy increases from 4 to $30-40 \mathrm{MJ}$ from low to high current and power, and the toroidal beta $\beta_{\mathrm{T}}$ increases from 3 to $12 \%$. Figure $28(e)$ shows the volume-average ion temperature $\left\langle T_{\mathrm{i}}\right\rangle$ increases from 3 to $5.5 \mathrm{keV},\left\langle T_{\mathrm{e}}\right\rangle$ increases from 2 to $4.8 \mathrm{keV}$, and the volumeaverage electron density increases from 0.6 to $2 \times 10^{20} \mathrm{~m}^{-3}$. Figure 28( $f)$ summarizes NBI power losses normalized to the total injected NBI power and shows that shine-through (ST) is the largest loss mechanism and decreases from $0.4-2 \%$ to $0.1 \%$ at higher current. As expected, the highest injection energy $(0.5 \mathrm{MeV})$ has the highest shine-through loss at the lowest current since this corresponds to the lowest target density and longest beam attenuation length at fixed Greenwald fraction. Charge exchange (CX) losses are highest for the lowest current and lowest $E_{\mathrm{NBI}}$ at low current, but these losses remain below $0.5 \%$. Bad-orbit losses (BO) are significantly lower than either shine-through or charge-exchange, and remain below $0.1 \%$ for all currents treated. Overall, these results indicate that with careful selection of equilibrium parameters, beam losses can be maintained below a few percent even at the $I_{\mathrm{P}}=2 \mathrm{MA}$, and for low $E_{\mathrm{NBI}}=0.1 \mathrm{MeV}$ at low current, even lower plasma currents should have acceptable beam losses. These results imply that if a suitable D-D target plasma of 2 MA (and possibly lower current) can be established, the ST-FNSF NBI system analyzed here can be used to ramp the plasma current to 7-8 MA, and this current level is suitable for confining alpha particles from D-T fusion reactions and for accessing fully non-inductive scenarios with 100-200 MW of fusion power.

An important consideration for the plasma current rampup phase is the ability to provide sufficient PF coil current to maintain the equilibrium and divertor power exhaust configuration. Figure 29(a) shows free-boundary equilibrium calculations of the plasma boundary shape and separatrix flux line into the divertor for a range of plasma currents. The lower plasma currents (2.3 MA, 4.3 MA, 7.6 MA) have current and pressure profiles from the TRANSP simulations of the 0.5/0.1/0.5/0.5 MeV (solid) NBI scenario for current ramp-up in D-D from figures 27 and 28. The highest current shown is the 11.6 MA D-T reference scenario discussed in section 2.2.3. As shown in the figure, all scenarios have divertor strike-points near the nominal design location $R_{\text {strike }} \approx 2.5 \mathrm{~m}$ and maintain (not shown) a total magnetic field-line angle of incidence in the range of $1-1.5^{\circ}$. Figure $29(b)$ shows the elongation and internal inductance for the plasma current scan consistent with the lower elongation at lower plasma current desirable for improved beam absorption. Figure 29(c) plots the PF coil current densities versus plasma current and shows that none of the lower plasma current scenarios have PF current densities exceeding the limits established in section 2.2.3. 

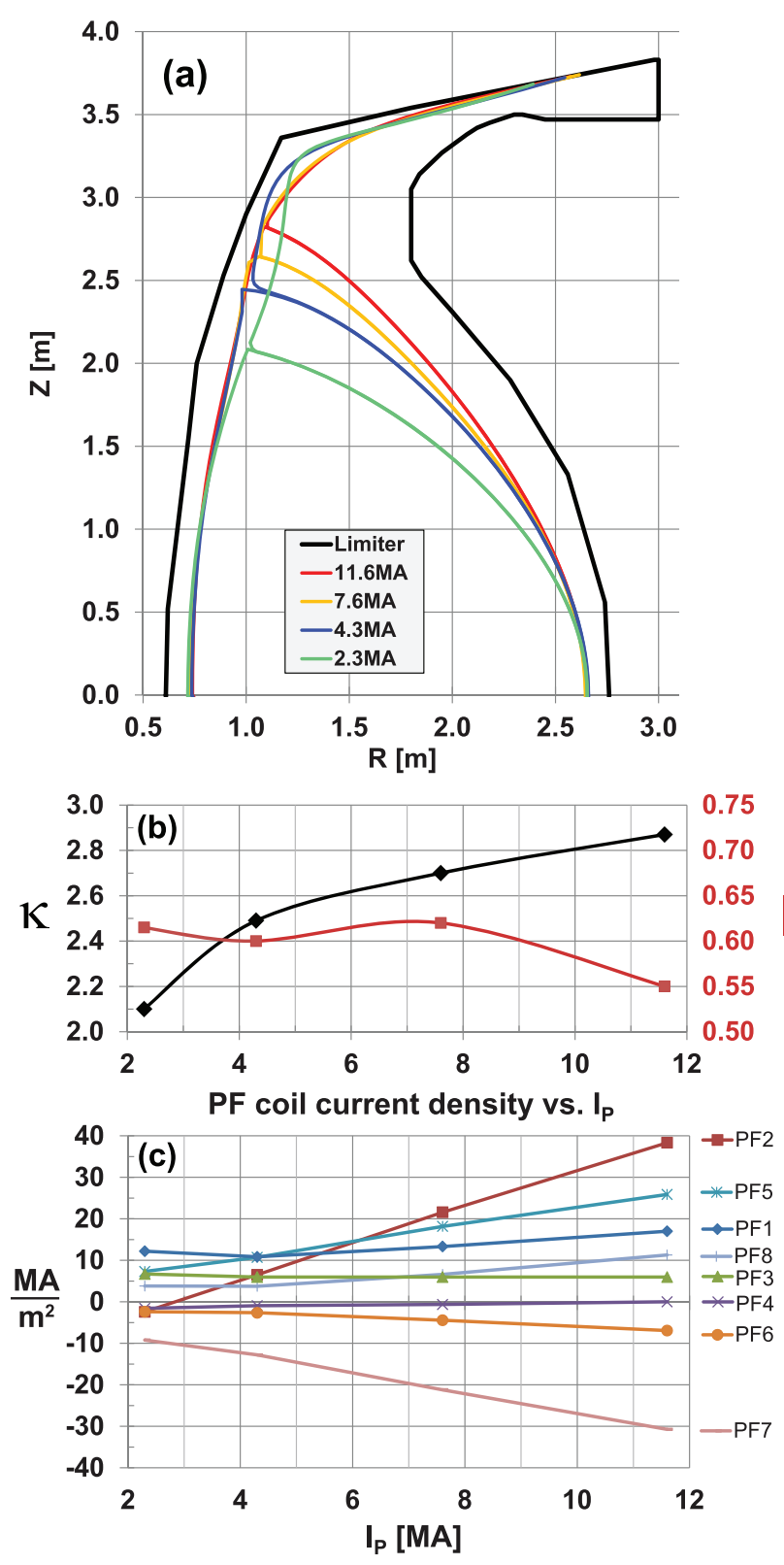

Figure 29. Free-boundary equilibrium calculations of the $(a)$ plasma boundary shape and separatrix flux line into the divertor, (b) elongation (and internal inductance), and (c) PF coil current densities all versus plasma current.

2.4.4. Plasma current formation. A remaining very important issue is generation of the initial target current of 1.5-2 MA needed for efficient NBI absorption for plasma current ramp-up as described in section 2.4.3. Plasma current initiation [160] is being investigated on a range of ST experiments world-wide and several options are being pursued [161]. NSTX-U start-up research will build upon NSTX results and continue to develop transient coaxial helicity injection (CHI) [162] as a means of creating a high-current target plasma of $400 \mathrm{kA}$ which extrapolates to approximately $2 \mathrm{MA}$ in an STbased FNSF [27].

A key concern for $\mathrm{CHI}$ in a nuclear environment is where and how to provide sufficient insulation to allow nominally axisymmetric voltage biasing to create the initial openfield-line plasma arc that ultimately generates a closed-flux tokamak plasma. Using proposed ST-FNSF blanket configurations described in more detail in section 4.2, analysis has already been performed to assess the viability of incorporating CHI voltage biasing within dual-coolant lead lithium (DCLL) blanket modules [163]. Figure 30 shows example concepts for incorporating electrical insulators in dual-coolant leadlithium (DCLL) blanket segments to support voltage biasing for CHI start-up in ST-FNSF. Figure 30(a) shows an 'NSTXlike' $[163,164]$ concept in which the blanket would be supported off the outer vessel using the green insulating plates and sandwiched between metal supports and the assembly bolted together using insulating bolts. Note that this figure shows a view of the blanket structure only. Figure $30(b)$ shows a 'DIII-D-like' [163, 165] concept in which a toroidal electrode would be installed on top of the blanket and separated from the rest of the blanket using the green toroidal insulator plate. Note that for this configuration the green insulating sections for insulating the piping system are not required. For both concepts, if $\mathrm{MgO}$ was used as the insulating material, the blanket module provides sufficient shielding to keep the radiation damage below an estimated damage limit of $10^{11} \mathrm{~Gy}$ for $\mathrm{MgO}[138,166,167]$.

\section{Device configuration and maintenance}

Several ST-FNSF equilibrium, divertor, PF and TF coil, centerstack, vessel, and blanket configurations and maintenance strategies were studied [168, 169] prior to down-selecting to the final configuration discussed here [170]. ST-FNSF engineering details relevant to the physics design are described for completeness and context.

\subsection{Central magnet concept}

As described in section 2.2, strong plasma shaping will be important for operating with sufficient stability margin for FNSF applications, and would be essential for accessing advanced operating modes with very high $\beta$ and fusion performance as shown in figure 12. In particular, achieving high triangularity $\delta$ is essential at high elongation to achieve high ST stability limits [20]. Further, increased triangularity generally increases peeling-ballooning limits and the achievable confinement in the H-mode pedestal region [171-174]. Achieving high- $\delta$ can be challenging in the ST configuration since at least one set of divertor poloidal field (PF) coils is required to be both inboard and close to the divertor $\mathrm{x}$-point. This is challenging in a nuclear environment since neutron damage to the PF coil insulation can substantially reduce the lifetime of the insulator and hence the coil.

Figure 31 shows a potential solution [168] to the divertor coil challenge in which PF coils in a 'Bitter plate' configuration are installed at the ends of the TF central rod and the centerstack shield and the toroidal field $\mathrm{Cu}$ conductor together help shield the PF coils from neutron damage. Unlike conventional magnets wound from coils of wire or cable, Bitter magnets are constructed of circular conducting metal plates with insulating spacers stacked in a helical configuration. Figure 31(a) 
(a)

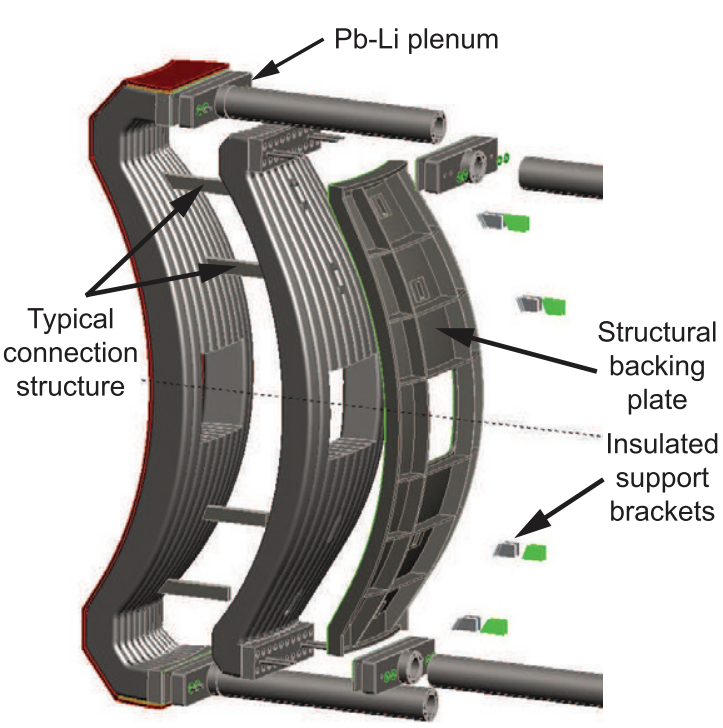

Blanket modules and piping insulated from rest of vessel

(b)

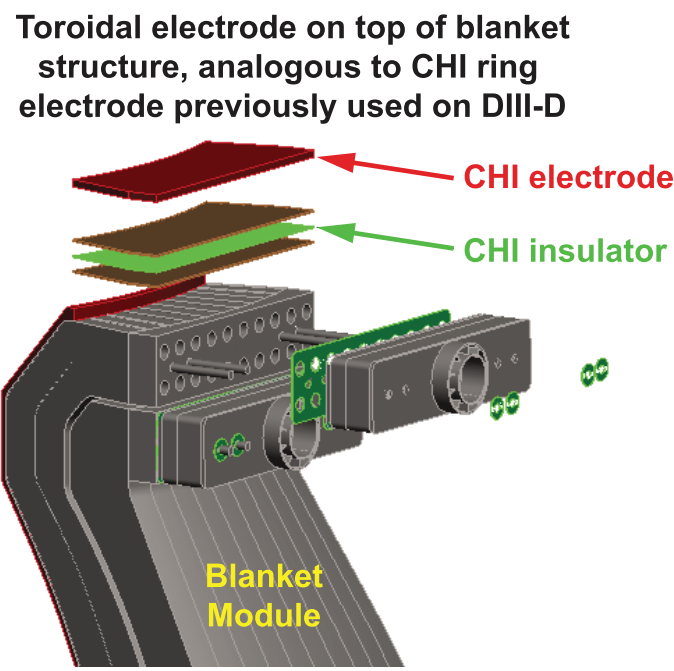

Figure 30. Possible concepts for incorporating electrical insulators in dual-coolant lead-lithium (DCLL) blanket segments to support voltage biasing for CHI start-up in ST-FNSF (figure reproduced with permission [163]). (a) Concept 1-NSTX-U-like. (b) Concept 2-DIII-D-like.

shows conceptually how Glidcop conducting plates might be plasma-sprayed with $\mathrm{MgO}$ to provide plate-to-plate insulation. Figure 31(b) shows how multiple plates could be stacked to form a coil with vertically aligned water coolant channels interfaced to centerpost coolant channels. Figure 31(c) shows the two coils structurally supported together in a cylinder, and figure $31(d)$ shows the location of the upper divertor PF coil cylinder in the end of the centerpost.

The single-turn toroidal field coil in the ST-FNSF centerstack [168] could use NSTX-U-like TF wedge segments [175] that are brazed or pressed-fit together (rather than insulated). Such wedging would enable NSTX-U-like grooves in the wedge face plus a welded tube for water cooling. Alternatively, the cooling channels could be formed from gun-drilled holes. Figure 32 shows a possible assembly/ fabrication sequence for the TF bundle starting from individual wedges/plates, to sub-assemblies/quadrants, to a full brazed center-post, to the final addition of vacuum vessel wall and inboard divertor plasma-facing components modules and divertor poloidal field coils.

\subsection{Arrangement and maintenance}

Figure 33 shows the general arrangement of the complete $R_{0}=1.7 \mathrm{~m}$ device. The central magnet is shown in orange as is the rest of the copper magnet system. The embedded Bitter plate $\mathrm{Cu}$ PF coils are shown at the top and bottom of the central magnet, and the horizontal outer TF legs have a felt-metal sliding joint with the central magnet. Similarly, there are felt-metal sliding joints between the horizontal and vertical TF outer legs. The TF coil leads for each individual outer leg exit the device at the bottom at large major radius. The centerstack, vacuum vessel (gray), blanketshield modules (red/pink), poloidal field coils (blue/gray), and torsional loads are all supported by the large external support structure (also gray) that surrounds other core components.

A key potential advantage of the ST for FNSF applications is modularity of the overall configuration due in large part to the demountability of the normally conducting $\mathrm{TF}$ coils. The cylindrical geometry of the ST configuration naturally lends itself to a vertical maintenance strategy. Figure 34 shows the ST-FNSF design enables independent removal of either the TF centerpost or the blanket system. This removal is accomplished by removal of the magnetic system upper beam structure, TF horizontal legs, and re-weldable vacuum vessel lid which contains a local cryostat containing any superconducting upper PF coils. The blanket feeder pipes are evident in the bottom of the vacuum chamber when the blanket system is removed. These pipes are fed by larger manifolds outside the core machine.

Figure 35 shows a possible ST-FNSF test-cell arrangement from several different vantage points. Figure 35(a) shows a side view of the core device (without centerstack) showing the JT60-SA NNBI layout modified to have 3 vertically stacked sources instead of 2 and up to $8.5 \mathrm{MW}$ per source, and with the beam cross-over point near the vacuum vessel boundary just behind the blankets to minimize the port size, fit between the outboard PF coils, and minimize the blanket aperture size required for the beams. Figure $35(b)$ shows a horizontal mid-plane slice cutaway view showing the central magnet, blankets, TF coils, support structure, but most importantly the layout of the 4 NNBI systems showing the various tangential access ports and also the placement of the beams (all aiming in the co-plasma-current direction) on opposite sides of the device to reduce the overall test-cell building volume. The four beams shown have representative tangency major radii of $R_{\tan }=170 / 210 / 230 / 240 \mathrm{~cm}=R_{0}+r_{\tan }$ where $r_{\tan } / a=0 / 0.4 / 0.6 / 0.7$, and this arrangement is very similar to that assumed for the TRANSP calculations shown in figure 25 . 


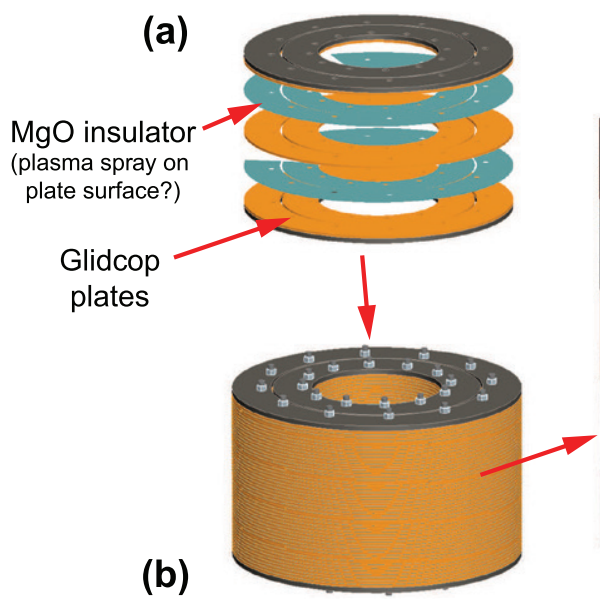

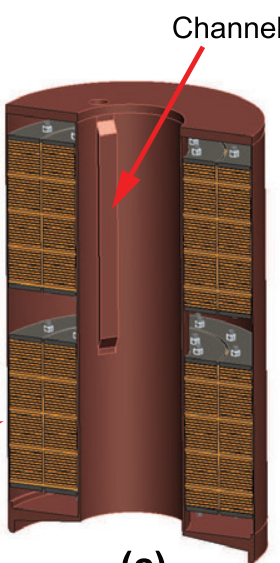

(c)

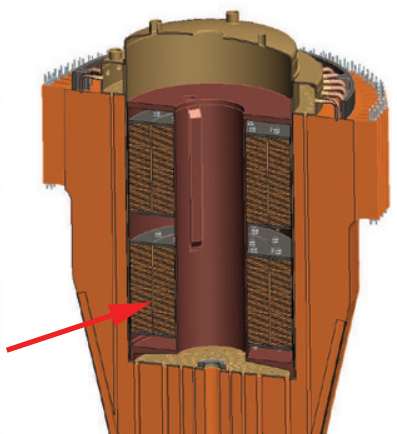

(d)

Figure 31. Bitter magnet concept for divertor poloidal field coils embedded in the ends of the toroidal field center-post.

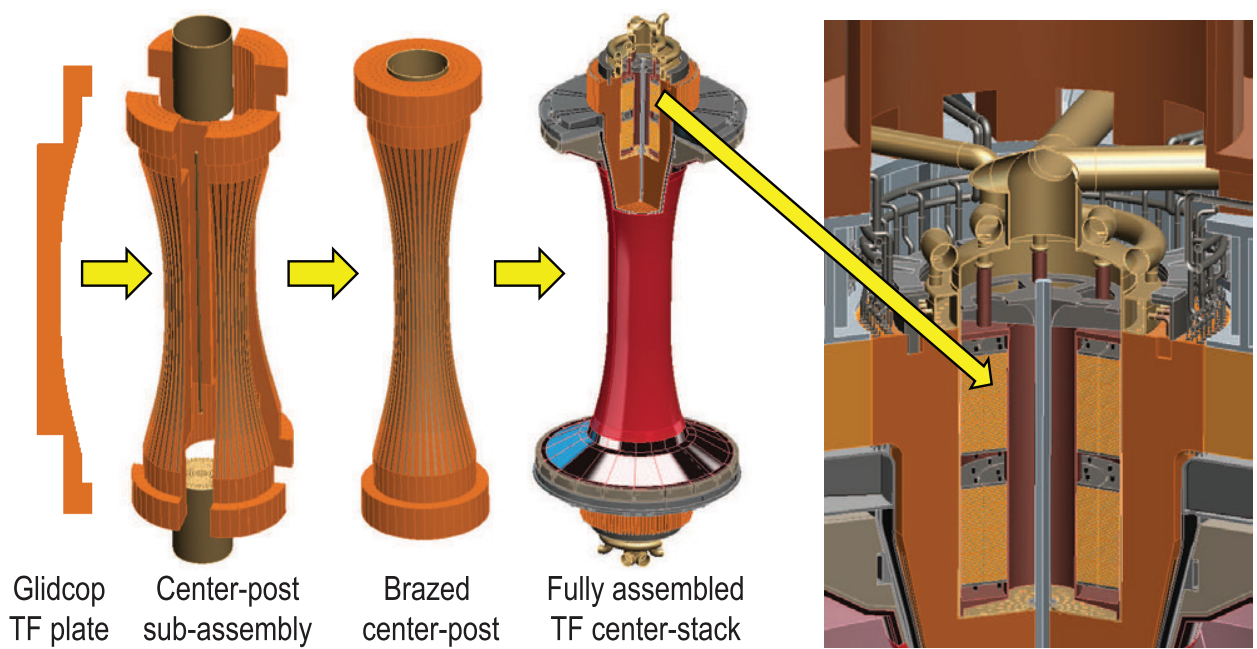

Figure 32. Center-stack assembly sequence from TF conductor plates to sub-assemblies to brazed centerpost conductors to a fully assembled centerpost.

The configuration shown in figure 35(b) has $12 \mathrm{TF}$ coils, and the NNBI ducts do fit in the space available, but additional space for future further optimization of tangency radius and/or additional duct shielding is feasible if the number of TF coils $N_{\mathrm{TF}}$ is reduced to $N_{\mathrm{TF}}=10$ as shown in figure 35(c). Because the major radius of the vertically straight TF outer legs $\left(R_{\mathrm{TF}}=5.85 \mathrm{~m}\right)$ is large compared to the plasma outboardmidplane separatrix radius $\left(R_{\mathrm{p}}=2.65 \mathrm{~m}\right)$, the toroidal field ripple [176, 177] $\delta \approx\left(R_{\mathrm{p}} / R_{\mathrm{TF}}\right)^{N_{\mathrm{TF}}}=0.036 \%$ for $N_{\mathrm{TF}}=10$ is small compared to values that might degrade edge rotation, pedestal confinement, or fast-ion confinement [177-180]. ST-FNSF options with $N_{\mathrm{TF}}=12$ would have very small ripple values $\delta \approx 7.5 \times 10^{-5}$, and either $N_{\mathrm{TF}}=10$ or 12 should be acceptable from a confinement physics standpoint.

Figure 35(c) shows the large test-cell area above the device core enabled by a vertical maintenance approach. This space would be used for assembly/disassembly of various large components including the blanket assemblies and TF centerstack after removal of the dome structure, upper TF horizontal legs, and vessel lid and upper PF cryo-stat. Figure 36 shows more details of the inside vacuum vessel boundary and upper re-weldable vacuum vessel seals that would enable this vertical maintenance approach. This figure also shows radial access ports for divertor module connection/maintenance and access to regions behind the blanket modules. Additional access for maintenance of smaller components inboard of the blankets would likely be provided via midplane radial ports via removal of materials test modules or test blanket modules.

\section{Neutronics calculations}

The coil and component layouts shown in figures 31-34 provide sufficient $\mathrm{CAD}$ detail to calculate important neutronics parameters such as shielding effectiveness and achievable tritium breeding ratio (TBR) [169]. The 3D CAD models have been coupled with the general Monte Carlo N-particle (MCNP) transport code [181] using the University of Wisconsin DAGMC code [182] to accurately represent the entire torus. Two ST device sizes have been analyzed for shielding and TBR as shown in table 1, and both devices include test blanket modules (TBMs) and a materials test module (MTM) to support the FNSF research and development mission [11]. For both sizes the assumed device lifetime is 20 years with an availability ranging from $10-50 \%$ with an 


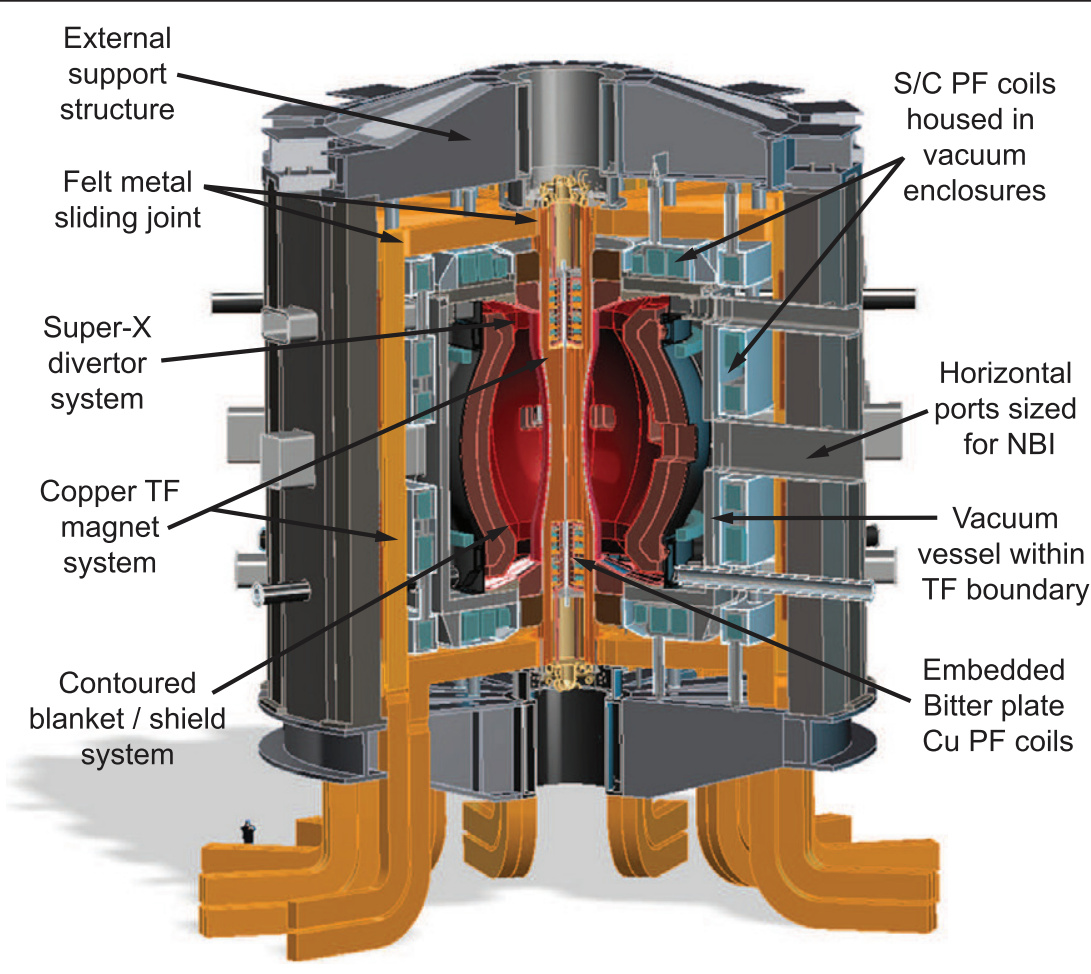

Figure 33. General arrangement for ST-FNSF showing TF and PF magnets, blanket modules, vacuum vessel, and external support structures.

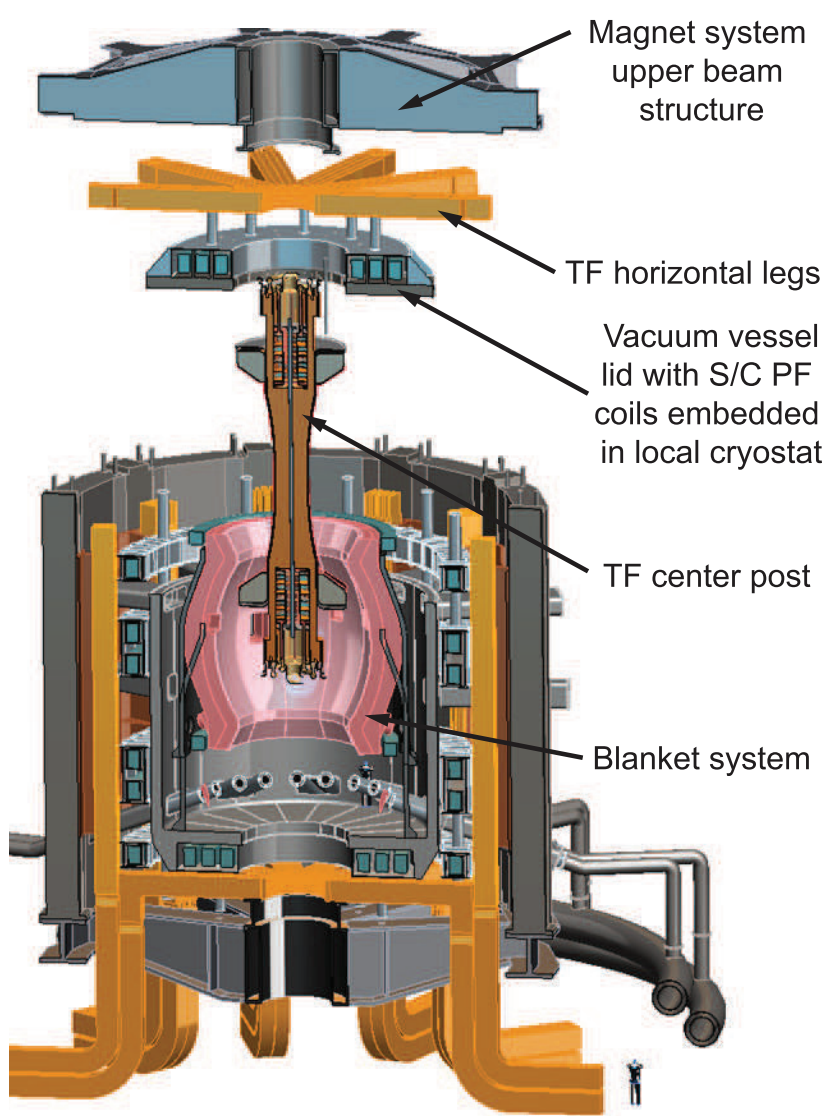

Figure 34. Vertical maintenance scheme showing removal of upper magnet system beam structure, TF horizontal legs, upper PF coils, TF centerpost, and blanket system. average value of $30 \%$ equivalent to 6 full power years (FPY) of operation. Shielding, blanket design, and tritium breeding calculations are described by El-Guebaly [169] and several details are repeated here.

The blanket concept of choice for the ST-FNSF base blanket is the dual-cooled lead-lithium (DCLL) [42] with ferritic steel (FS) structure, LiPb breeder/coolant, SiC flow channel inserts (FCI) and helium coolant. Such 'base' blankets would operate initially with reduced coolant temperature (e.g. LiPb and $\mathrm{He}$ inlet/outlet temperatures of $350{ }^{\circ} \mathrm{C} / 450{ }^{\circ} \mathrm{C}$ ). This concept requires FCI to serve as thermal and/or electric insulators [42]. If the more advanced $\mathrm{SiC}$-based FCIs (that allow high $\mathrm{LiPb}$ exit temperature of $700{ }^{\circ} \mathrm{C}-800{ }^{\circ} \mathrm{C}$ ) cannot be developed and qualified within the FNSF timeframe, lowtechnology sandwich-like inserts made of a FS/alumina/FS multilayer could be employed for the base blanket. Other features of the first-generation (GEN-I) base blanket include:

1. Low-activation FS structure operating at $400{ }^{\circ} \mathrm{C}-500{ }^{\circ} \mathrm{C}$

2. He-cooled first-wall (FW) and blanket structure

3. More uniform FW and blanket structure temperature to minimize thermal stresses

4. FCI made of $\mathrm{SiC}$, if available, or sandwich-like FS/ alumina/FS

5. Beryllium multiplier to enhance the breeding, if needed.

The inclusion of TBM ports in ST-FNSF offers the opportunity to test a wide spectrum of blanket concepts in an environment representative of DEMO or power plant. This includes conventional GEN-I blanket technologies (ceramic breeders and liquid breeders with FS structure operating at 

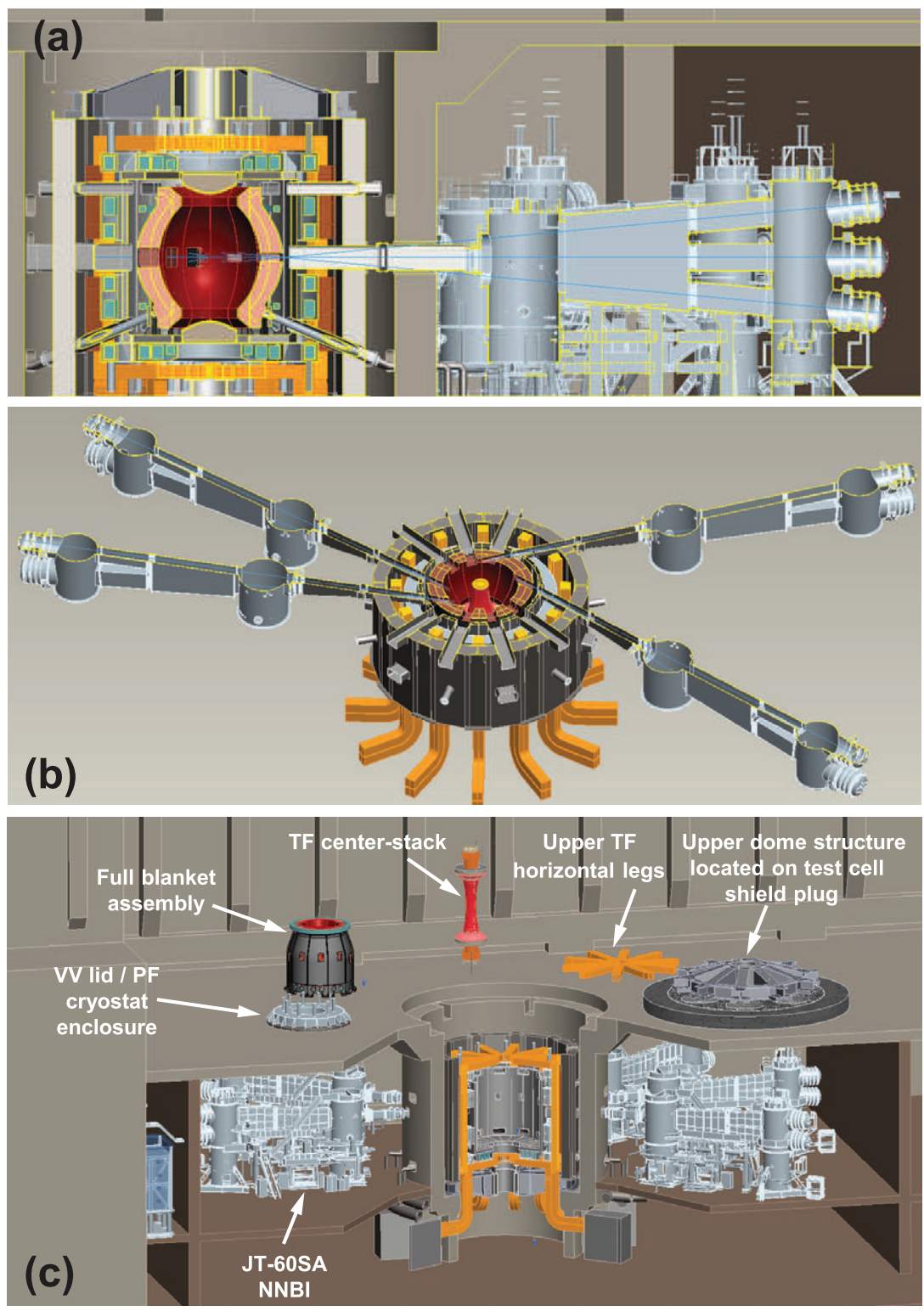

Figure 35. ST-FNSF test-cell layout showing $(a)$ vertically-stacked NNBI beam-line aiming, $(b)$ toroidal layout of NNBI systems, and (c) upper hot-cell above main vessel chamber.

$400{ }^{\circ} \mathrm{C}-500{ }^{\circ} \mathrm{C}$ ), moderately aggressive concepts (GEN-II blanket such as DCLL with $\mathrm{LiPb}$ exit temperature of $700{ }^{\circ} \mathrm{C}-$ $800{ }^{\circ} \mathrm{C}$ ), and advanced blanket concepts (GEN-III blanket with $\mathrm{SiC} / \mathrm{SiC}$ composite structure operating at up to $1000^{\circ} \mathrm{C}$ ). A staged blanket testing strategy would allow the ST-FNSF to start with a lower-technology and higher reliability base blanket, followed by a stepwise upgrade of the base blanket using results obtained from the TBMs to ultimately validate the characteristics and features of more advanced blankets [169].

\subsection{Shielding and neutron irradiation distribution}

Figure 36 shows neutron dose calculations at the corners of the PF coil regions in both the TF centerstack and also behind the divertor exhaust region for the $R_{0}=1.7 \mathrm{~m}$ configuration parameters from table 1 . Assuming $\mathrm{MgO}$ insulation of $\mathrm{Cu}$ conductors for the divertor PF coils (PF1 and 2) in the TF in the centerstack, the peak neutron dose $\left(6 \times 10^{9} \mathrm{~Gy}\right)$ is well below (by a factor of 16) the present best value of the allowable limit of $10^{11}$ Gy $[138,166,167]$. Thus, for the PF coils in the ends of the TF centerstack, the $\mathrm{Cu}$ of the TF bundle not only provides the conducting path for the TF coil current but also provides shielding for the inner-most divertor PF coils. This factor of 16 shielding margin is also adequate to shield the divertor PF coils in the smaller $R_{0}=1 \mathrm{~m}$ ST-FNSF. The top and bottom divertor region PF coils (PF3, 4, 5) have doses below $2 \times 10^{8} \mathrm{~Gy}$ and thus $\mathrm{MgO}$-insulated $\mathrm{Cu}$ coils should have insulator radiation damage values far below $10^{11} \mathrm{~Gy}$. The most outboard PF coils (PF6, 7, 8) that are shielded by both the blankets and the vessel have radiation and heating below limits for superconducting coils and are assumed to be superconducting.

To reduce resistive power consumption it would be advantageous if PF coils 3, 4, and 5 were also superconducting. Neutronics calculations indicate this may be possible using $\mathrm{Nb}_{3} \mathrm{Sn}$ superconductors [183-186]. First, the peak nuclear 


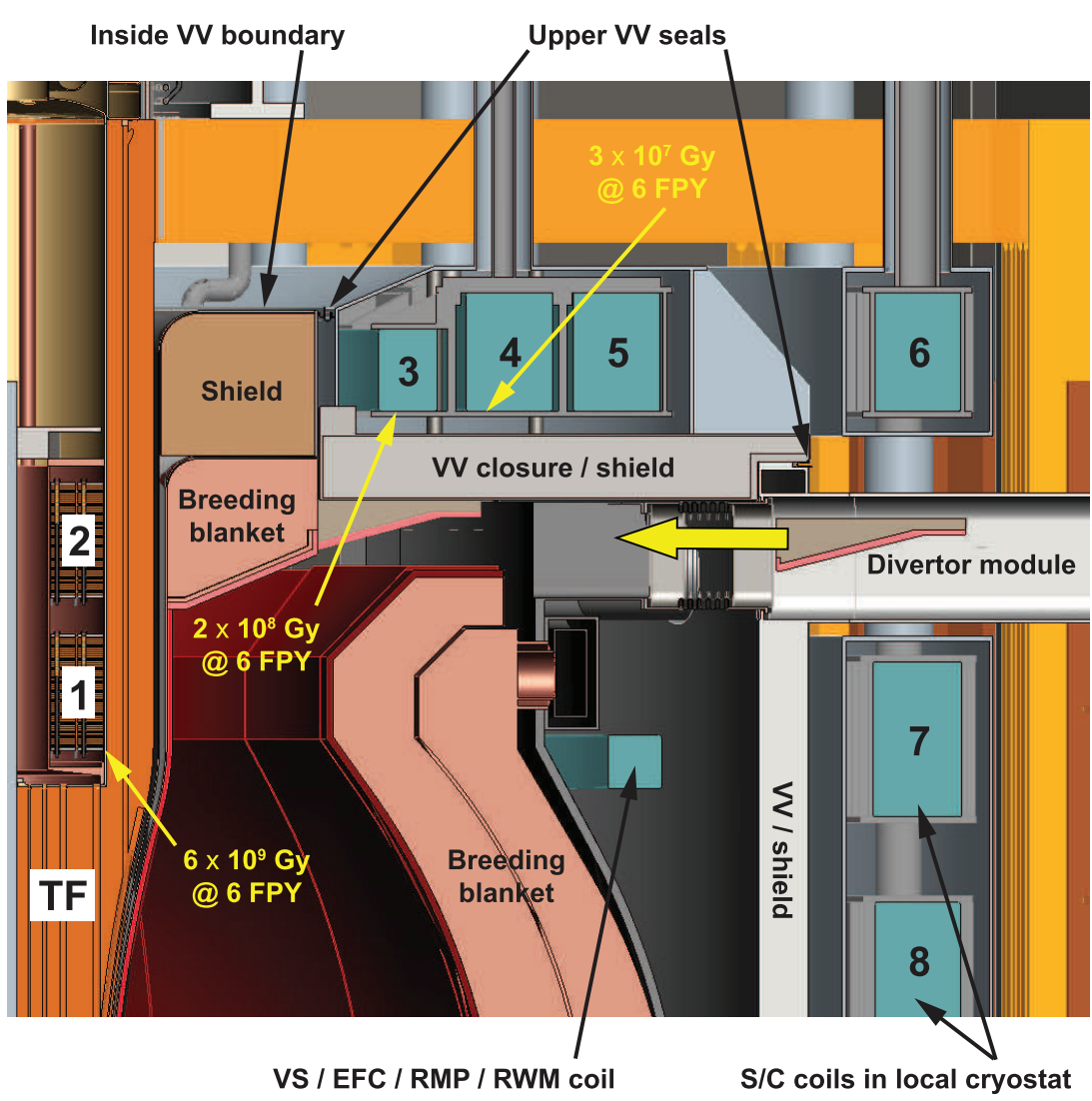

Figure 36. Radial maintenance ports for divertor modules (thick yellow arrow), vacuum vessel boundary and re-weldable seal details, and in-vessel radiation dose values at various inboard divertor field coil positions (thin yellow arrows).

Table 1. Parameters for $R_{0}=1.7 \mathrm{~m}$ and $1 \mathrm{~m}$ ST-FNSF devices for neutronics analysis.

\begin{tabular}{lll}
\hline Major radius $(\mathrm{m})$ & 1.68 & 1.00 \\
Aspect ratio & 1.75 & 1.75 \\
Fusion power $(\mathrm{MW})$ & 162 & 62 \\
${\text { Avg. } \Gamma_{\text {neutron }}\left(\mathrm{MW} \mathrm{m}{ }^{-2}\right)}$ ) & 1 & 1 \\
Number of TF coils $^{\text {Number of TBM ports }}$ & 12 & 10 \\
Number of MTM ports & 1 & 4 \\
Number of NBI ports & 4 & 1 \\
\hline
\end{tabular}

heating of the PF3 coil casing for the $R_{0}=1.7 \mathrm{~m}$ configuration is $2.2 \mathrm{~mW} \mathrm{~cm}^{-3}$ compared to a typical limit of $2 \mathrm{~mW} \mathrm{~cm} \mathrm{~cm}^{-3}$ (and $1 \mathrm{~mW} \mathrm{~cm}^{-3}$ for the winding pack). Further, the peak PF3 dose to cyanate ester epoxy insulators in the superconducting cables is $1.1 \times 10^{8}$ Gy compared to a limit of up to $4.0 \times 10^{8}$ Gy [187]. However, the peak fast neutron fluence $\left(E_{\mathrm{n}}>0.1 \mathrm{MeV}\right)$ to PF3 at 6 FPY is $1.2 \times 10^{23} \mathrm{~nm}^{-2}$. This is roughly one order of magnitude above the generally accepted ITER design limit [188] of $1 \times 10^{22} \mathrm{~nm}^{-2}$ for ternary or quaternary strand [189] for which there is only small degradation in critical temperature and current density. However, if binary conductor could be used, or if 20-30\% degradations in critical temperature and current density in ternary/quaternary strand could be tolerated, operation up to $10^{23} \mathrm{~nm}^{-2}$ may be possible [185] — in particular if more recently developed wires such as restack-rod-processed (RRP) or powder-in-tube (PIT) enable the critical current density to be increased at high radiation doses [190]. Thus, for the $R_{0}=1.7 \mathrm{~m}$ configuration it appears PF4-8 could all be superconducting while PF3 might be superconducting given increased radiation tolerance and/or shielding. These calculations also indicate that PF3 and possibly PF4 cannot be superconducting in the less-well-shielded $R_{0}=1 \mathrm{~m}$ device.

Figure 37 shows the dpa distribution throughout the structure of the $R_{0}=1.7 \mathrm{~m}$ configuration and indicates the expected highest radiation location of the outboard midplane. The peak outboard dpa of $15.5 \mathrm{dpa} / \mathrm{FPY}$ implies $93 \mathrm{dpa}$ total damage to the outboard first-wall for 6 FPY of operation. Among other effects, high temperature He embrittlement can cause intergranular fracture at low stresses, particularly for doses $>10$ dpa (He concentrations $>100$ appm) and temperatures above 0.5 times the melting temperature [191, 192] although some ODS steels may be usable for up to $20 \mathrm{dpa}$ and $200 \mathrm{appm} \mathrm{He}$ concentrations $[193,194]$. Thus, the total dpa for 6 FPY level is 9 times the damage limit for 10 dpa-capable ferritic steel and calls for the further development of more radiation resistant ferritic steel structures that can handle $100 \mathrm{dpa}$ or more. The TBMs and MTMs at the outboard midplane of the ST-FNSF are clearly subject to a fusion-relevant nuclear environment to help develop and test materials and components for fusion power production applications [169].

Finally, figure 37 also shows that the inboard mid-plane $\mathrm{Cu}$ TF magnet peak radiation damage is $10-12 \mathrm{dpa} / \mathrm{FPY}$. However, irradiation at temperatures below $150{ }^{\circ} \mathrm{C}$ causes hardening in pure copper and alloys. This hardening is accompanied by severe embrittlement $[195,196]$ in dispersion strengthened and precipitation hardened alloys such as 


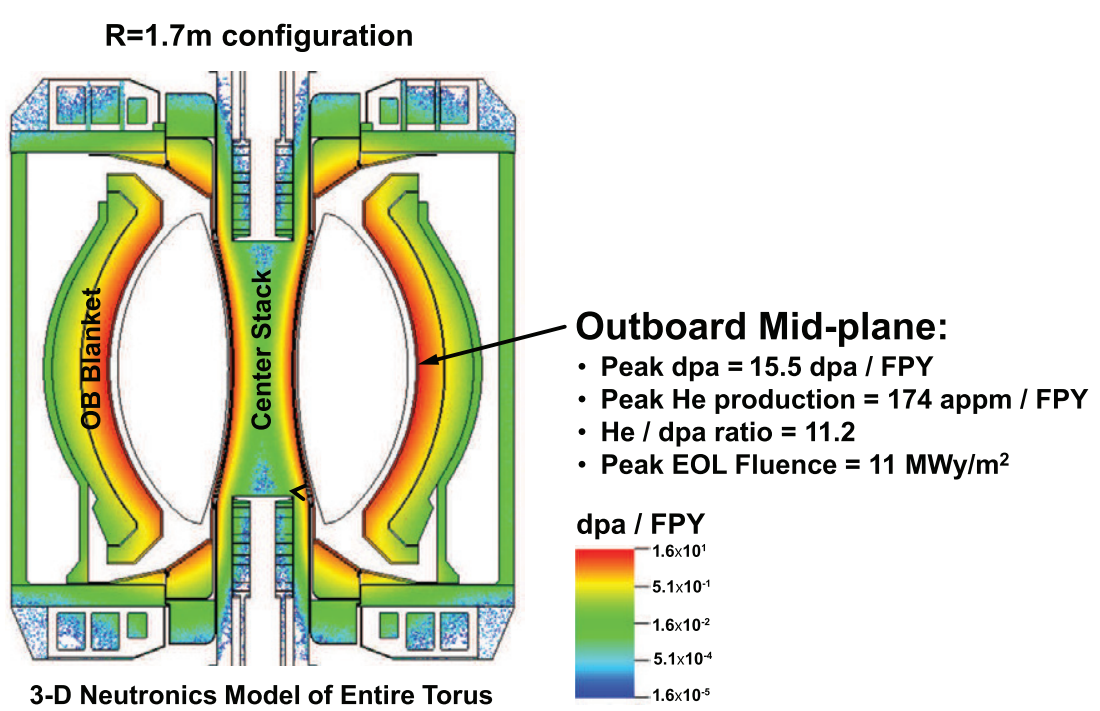

Figure 37. Distribution of dpa for $R_{0}=1.7 \mathrm{~m} \mathrm{ST-FNSF} \mathrm{device.}$

$\mathrm{CuCrZr}$. The uniform elongation generally decreases to less than $1 \%$ even at doses as low as $0.01-0.1 \mathrm{dpa}$. Tensile stresses in the TF central conductor must therefore be minimized [6], and felt-metal sliding joints between the vertical and horizontal TF legs of ST-FNSF are chosen to accommodate TF vertical expansion to maintain stresses within allowable limits $[168,170]$.

\subsection{Tritium breeding}

Achieving tritium self-sufficiency is an important requirement for a fusion system, and the relevant parameter for selfsufficiency is the tritium breeding ratio (TBR), i.e. the ratio of tritium bred to tritium consumed. A particular issue for smaller ST-FNSF devices is that it will likely be more difficult to achieve TBR $=1$ since a higher fraction of in-vessel surface area must be dedicated to auxiliary heating ports and test modules. To begin to analyze the dependence of TBR on device size, the TBR for a range of blanket configurations has been computed for the $R_{0}=1.7 \mathrm{~m}$ FNSF device assuming an idealized (i.e. nominally axisymmetric and homogeneous) blanket and a conventional divertor configuration with little/ no breeding in the top and bottom divertor regions. Figure 38 shows these blanket configurations (light blue) and lists a TBR value for each. Figure 38(a) shows that the TBR of a straight blanket with a height comparable to the plasma height has $\mathrm{TBR}=0.8$ which is significantly below 1 due to losses to the magnets and external components. Additional calculations (not shown) for this blanket vertically extended to the upper height of the vacuum boundary increase the TBR to near 1 , but this does not leave room at the top and bottom of the vessel for divertor pumping or maintenance or other manifolds. Figures $38(b)$ and $(c)$ show that either the additional top/bottom blanket modules or having a conformal blanket can increase the TBR to 1.05 . Figure $38(d)$ extends the conformal blanket to the top and bottom of the vessel. The $3 \mathrm{D}$ model of the $R_{0}=1.7 \mathrm{~m}$ design indicates that the slots in the ends of the blankets for divertor access and maintenance would modify the TBR to be approximately mid-way between the values in figures $38(c)$ and $(d)$, i.e. the effective $\mathrm{TBR}=1.073$. Figure 38(e) shows that the inclusion of stabilizing shells (for suppressing plasma vertical instability) reduces TBR by approximately 0.03 , and figure $38(f)$ shows that 10 midplane penetrations of $0.36 \mathrm{~m}^{2}$ each (typical of the JT-60SA NNBI beam port size) would further reduce the TBR by 0.05 . Thus, the approximate TBR for the $R_{0}=1.7 \mathrm{~m}$ beamdriven ST-FNSF device is $1.073-0.03-0.05=0.993$, i.e. very close to 1 . This nominally axisymmetric and homogeneous blanket analysis indicates that $R_{0}=1.7 \mathrm{~m}$ is very close to the threshold for tritium self-sufficiency, and that smaller devices with relatively larger blanket penetrations will have difficulty achieving $\mathrm{TBR}=1$ even under idealized conditions.

More detailed fully 3D TBR calculations have also been carried out for the final $R_{0}=1.7 \mathrm{~m}$ ST-FNSF configuration as shown in figure 39. No approximations have been utilized in the blanket neutronics analysis, and many configuration details are retained including: (1) $2 \mathrm{~cm}$ wide assembly gaps between toroidal sectors, (2) Internals of two outboard (OB) DCLL blanket segments modeled in great detail, including the first-wall (FW), side, top/bottom, and back walls, cooling channels, and SiC FCI, (3) $2 \mathrm{~cm}$ thick $W$ vertical stabilizing shells between OB blanket segments, and (4) FS port walls for test blanket/materials test modules (TBM/MTM) and NNBI. As seen in figure 39, the inner-most radial segment of the outboard blanket provides a TBR of 0.81, while the outer-most segment provides 0.15 for a total outboard blanket TBR of 0.96. Thus, to achieve TBR $>1$ even with no penetrations or ports, additional breeding regions are needed. A key advantage of the long-legged super-X/snowflake divertor is that the divertor strike-point region can be moved to larger major radius away from the relatively high neutron flux regions at the top and bottom ends of the centerstack. By breeding in these top/bottom end regions, the total TBR can be increased by an additional 0.07 for a total of 1.03 .

Figure 40 shows the impact of including a range of midplane ports including 4 TBMs and 1 MTM with blanket frontface areas of $0.9 \mathrm{~m}^{2}$ each, and penetrations for the negative neutral beams with aperture areas of $0.4 \mathrm{~m}^{2}$ perpendicular 
Straight blanket

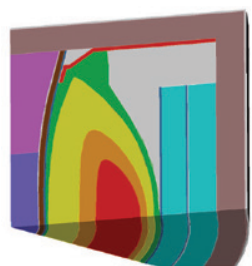

(a)

\section{Straight blanket with flat top}

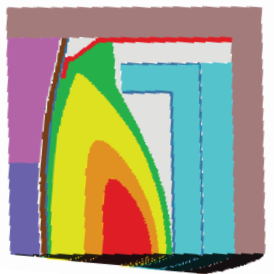

(b)

\section{Conformal blanket}

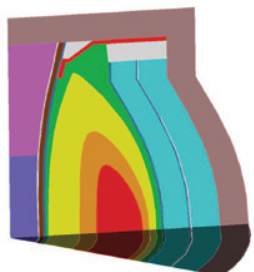

(c)
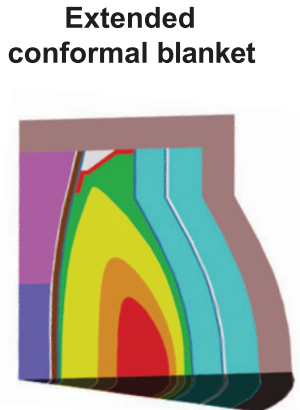

(d)

\section{Extended conformal blanket with $3 \mathrm{~cm}$ thick stabilizing shell}

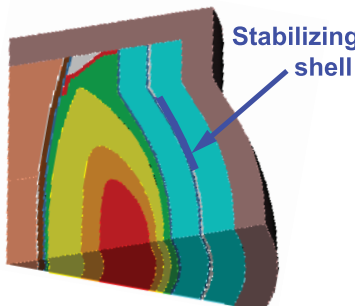

(e)

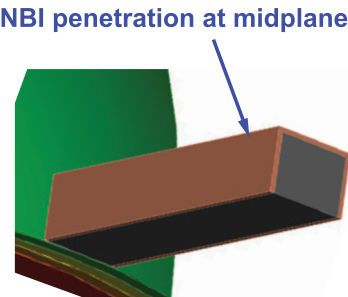

(f)

Figure 38. Calculated TBR for several nominally axisymmetric (except for case $f$ ) blanket configurations for $R_{0}=1.7 \mathrm{~m} \mathrm{ST-FNSF}$. (a) $\mathrm{TBR}=0.8$. (b) $\mathrm{TBR}=1.047$. (c) $\mathrm{TBR}=1.046 .(d) \mathrm{TBR}=1.1$. ( $) \mathrm{TBR}=1.07 .(f) \mathrm{TBR}=1.02$ with 10 NBI penetrations.

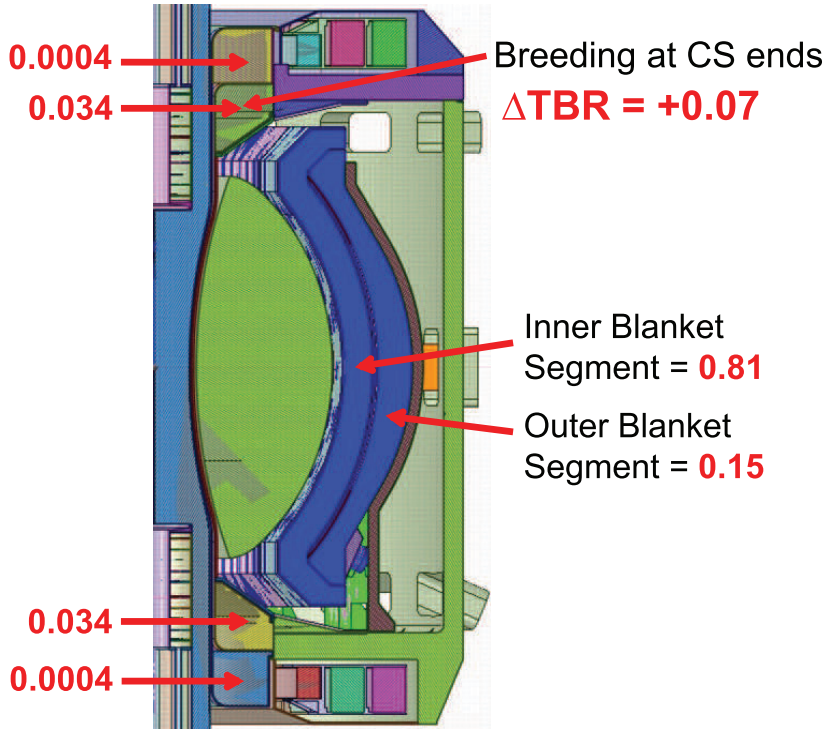

Total TBR $\sim 1.03$ with no penetrations or ports (heterogenous outboard blanket)

Figure 39. Calculated TBR for various components of fully 3D model of $R_{0}=1.7 \mathrm{~m}$ FNSF.

to the beam-line. As shown in the figure, the TBMs provide breeding nearly as efficiently as the DCLL base blanket with an overall TBR reduction of only $1 \%$ ( $0.25 \%$ per TBM). In contrast, the MTM does not provide breeding which leads to a TBR reduction of $2 \%$ per port. Lastly, each of the 4 NNBI ports is sized to support $20 \mathrm{MW}$ of NBI power with a perpendicular aperture area of $0.4 \mathrm{~m}^{2}$ for an average port power density of $50 \mathrm{MW} \mathrm{m}{ }^{-2}$ [197]. As discussed in section 2.4.2, different power-splits per port (5/25/25/25 MW) may ultimately be required to optimize the steady-state current drive profile, so specifying each of the 4 NNBI ports have the same perpendicular aperture could introduce small differences in the TBR calculations. Nevertheless, the total multi-port NNBI aperture area is the same $\left(1.6 \mathrm{~m}^{2}\right)$ for the same total NNBI injected power of $80 \mathrm{MW}$. The total TBR reduction from all 4 NNBI ports is $3 \%$, i.e. an average of $0.75 \%$ per NNBI. Including all 4 TBMs, 1 MTM, and 4 NNBI ports results in an overall TBR of 0.97 .

It is highly desirable to demonstrate tritium self-sufficiency in an FNSF device, and the calculated TBR for the $R_{0}=1.7 \mathrm{~m}$ ST-FNSF of 0.97 is very close to unity. Several ideas/options have been identified to further increase TBR to values above 1 , and these ideas are shown in figure 41 . These options include: adding to the top/bottom PF coil shield a thin breeding blanket (estimated to increase TBR by $\approx 3 \%$ and requiring a $\mathrm{Cu}$-conductor PF3), reducing the size of the opening to the divertor to reduce neutron leakage (and requiring a narrower range of $l_{\mathrm{i}}$ ), having a uniform outboard blanket thickness ( $1 \mathrm{~m}$ thick everywhere versus $0.85 \mathrm{~m}$ at top/bottom), increasing the $\mathrm{LiPb}$ flow channel dimensions and reducing cooling channels and flow-channel inserts within the outboard blanket (thermal analysis needed to confirm), and/or adding a thin breeding region to the inboard vacuum-vessel. It is expected that some combination of these options will enable achievement of TBR $\geqslant 1$ for the $R_{0}=1.7$ m configuration. In contrast, the TBR for the $R_{0}=1 \mathrm{~m}$ configuration (with only 3 NBI ports) is found to be 0.88 which is far enough below 1 that even if the options to increase TBR shown in figure 41 are exploited, the TBR will very likely still be below 1 . Despite 


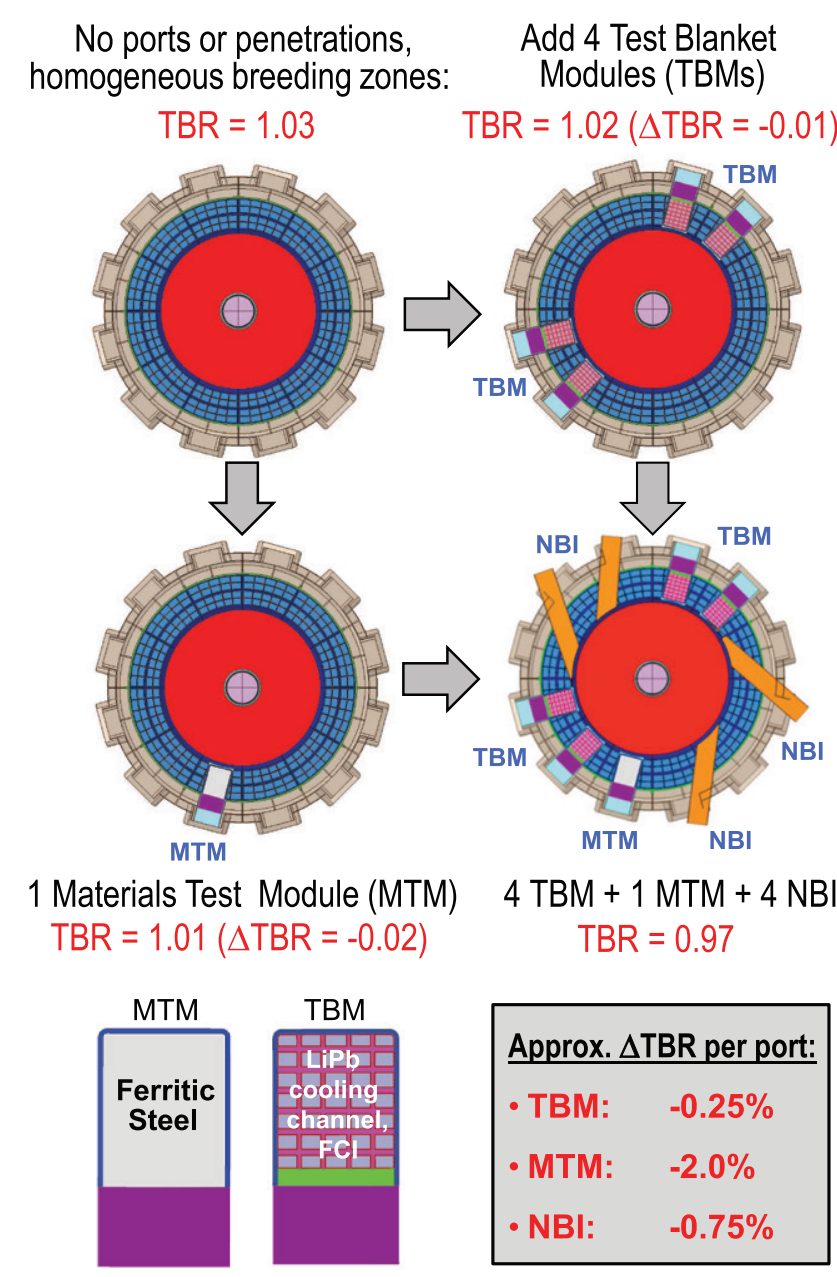

Figure 40. Calculated TBR versus type of mid-plane penetrations for $R_{0}=1.7 \mathrm{~m}$ ST-FNSF configuration.

this (expected) inability to achieve TBR $\geqslant 1$ in the relatively small $R_{0}=1 \mathrm{~m}$ device, TBR of 0.88 is still substantial and would reduce the required external supply of $\mathrm{T}$ by a factor of $1 /(1-\mathrm{TBR}) \geqslant 8$ relative to not breeding any tritium.

Figure 42 shows the spatial distribution of the $\mathrm{T}$ production in the blanket and TBMs for the $R_{0}=1 \mathrm{~m}$ device. Note that despite the significant area opened by the NBI penetrations, the very tangential injection allows streaming neutrons to be captured at the back of the blanket, thereby offsetting the reduction in TBR relative to what would be expected based on blanket front-face-aperture area (i.e. normal to the blanket face not the beam-line) scalings alone. This analysis also indicates the importance of having sufficiently thick outboard blankets to maximize tritium breeding in blanket modules near the tangential beam ducts. For the $R_{0}=1 \mathrm{~m}$ device it will be necessary to purchase $0.4-0.55 \mathrm{~kg}$ of T/FPY from outside sources at a cost of $30-170 \mathrm{~kg}^{-1}$ of T [198] (in 2015 USD) implying a total cost of $12-94 \mathrm{M} / \mathrm{FPY}$. Since the expected average duty factor is 0.3 , the estimated annual average cost for $\mathrm{T}$ is $4-28 \mathrm{M}$ per year which is likely an acceptable operating cost for a major nuclear device and associated program. However, there is uncertainty in relying on external sources to supply $\mathrm{T}$ fuel $(\approx 3 \mathrm{~kg}$ over $6 \mathrm{FPY})$ for such a program.

Finally, Figure 43 shows a side-by-side and to-scale summary comparison of the $R_{0}=1.7 \mathrm{~m}$ and $1 \mathrm{~m} \mathrm{ST-FNSF}$ configurations showing the device sizes, expected TBR values, and the TBM, MTM, and NBI port layouts. It is expected the $R_{0}=1 \mathrm{~m}$ device would have lower electricity and capital cost but higher tritium consumption and purchase costs (assuming the $R_{0}=1.7 \mathrm{~m}$ device can achieve TBR $=1$ ). Details such as the port layout could influence overall device cost in important ways. For example, the smaller $R_{0}=1.0 \mathrm{~m}$ device may require beam injection on only 1 side of the device, and this could reduce the overall size and cost of the building housing the FNSF device. Additional analysis is required to assess device size and cost trade-offs in more detail, and such analysis is beyond the scope of the present work.

\section{ST-based pilot plants using HTS TF magnets}

The $R_{0}=1$ and $1.7 \mathrm{~m}$ ST-FNSF devices operating with $B_{\mathrm{T}}=3 \mathrm{~T}$ at $R_{0}$ require 15 and $25.5 \mathrm{MA}$ of total single-turn TF rod current, respectively. Even with 12 TF legs and independent power supplies for each leg, large power supply currents of 1.25 to $2.1 \mathrm{MA}$ would be required to power the TF magnets. Using conventional rectifier power supplies of $125 \mathrm{kA}$ each, figure 44 shows a possible power supply arrangement and building area/footprint required to power the $R_{0}=1.7 \mathrm{~m}$ ST-FNSF TF coil [170]. While vertical stacking of the power supply sections could likely reduce the power supply distance to the TF coils, the overall floorspace area is large and comparable in size to the main tokamak building. More efficient and compact power supplies based on homopolar generators [199-202] could potentially significantly reduce this footprint but require significant research and development. Including the TF outer legs and current leads, the resistive power dissipation could be in the range of 150 to $250 \mathrm{MW}$ for the $R_{0}=1$ to $1.7 \mathrm{~m} \mathrm{ST-FNSF}$ devices. This translates to 50-80M USD for cost of electricity per year at $30 \%$ duty factor and 0.1 $\mathrm{kWh}$. Further, the higher recirculating/dissipated power of a copper toroidal field coil increases the fusion power required to achieve $Q_{\text {eng }}=1$ in ST-based pilot plants [3].

The obvious option to reduce power loss in the ST TF magnet system is to use superconductors [203, 204]. However, the additional inboard shielding required to reduce nuclear heating and radiation damage to acceptable levels combined with the typical need for inboard breeding for $\mathrm{TBR} \approx 1$ all tend to make larger major radius and/or higher aspect ratio devices more attractive for fusion power production. For reference, the maximum effective current density in the $R_{0}=1.7 \mathrm{~m}$ TF magnet $\mathrm{Cu}$ conductor is approximately $27 \mathrm{MA} \mathrm{m}^{-2}$ at the smallest major radius section of the centerstack. Thus, in order for the use of superconductors to be competitive at lower-A, the effective current density must be significantly higher to provide space for additional shielding. Recent advances in HTS magnet technology potentially capable of accessing much higher current density (see section 2.2.3) of up to $70 \mathrm{MA} \mathrm{m}^{-2}$ (and possibly higher) combined with operation at higher temperature for associated reductions in refrigeration power in the presence of increased nuclear heating [205] may make it possible for lower aspect ratio superconducting configurations to still be advantageous for FNSF and pilot plant applications. The higher field capability of HTS is also advantageous in 


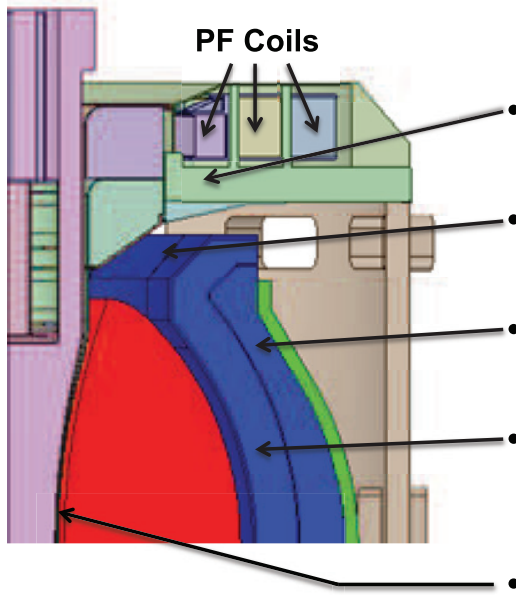

Options to increase TBR:

Add to PF coil shield a thin breeding blanket $(\triangle \mathrm{TBR} \sim+3 \%)$

Smaller opening to divertor to reduce neutron leakage

Uniform OB blanket (1m thick everywhere; no thinning)

Increase LiPb flow channel dimensions and reduce cooling channels and FCls within blanket (need thermal analysis to confirm)

Figure 41. Options for increasing tritium breeding ratio for $R_{0}=1.7 \mathrm{~m}$ device.

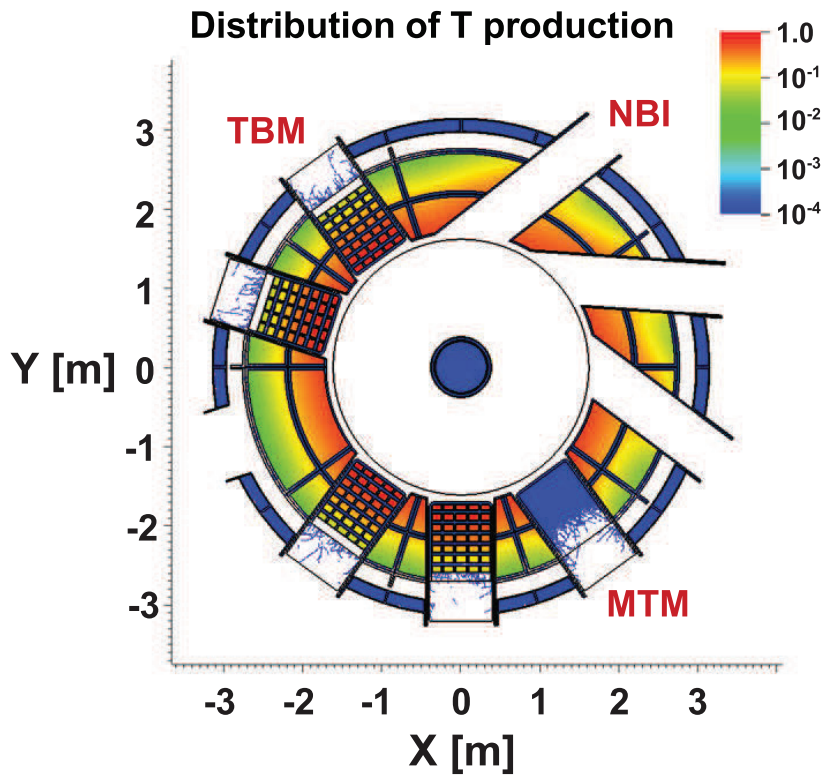

Figure 42. Calculated breeding distribution for $R_{0}=1.0 \mathrm{~m}$.

reducing the device size and/or increasing the MHD stability margin of the operating point as highlighted in the proposed ARC device [206] with $A=2.9$. These considerations motivate studies of projected device performance versus aspect ratio when high-field and high-current-density rare earth barium copper oxide (REBCO) superconducting HTS toroidal field magnets are utilized. Only with such studies is it possible to determine any relative advantages and disadvantages of low-A HTS-based FNSF/pilot plants.

\subsection{Aspect ratio scalings for tokamak pilot plants}

As discussed in section 2.1.1, the assumed $\beta_{\mathrm{N}}$ and $\kappa$ dependence on aspect ratio plays a very strong (approximately quartic) role in the projected fusion performance of highbootstrap-fraction ST and AT scenarios since $P_{\mathrm{f}} \propto \epsilon\left(\beta_{\mathrm{N}} \kappa B_{\mathrm{T}}\right)^{4}$. The profile-optimized no-wall beta limit is a useful guide for parameterizing $\beta_{\mathrm{N}}(\epsilon)$ and is more conservative than ideal-wall stability [47] scalings. A reasonable fit to the computed nowall limit at $f_{\mathrm{BS}} \approx 50 \%[20]$ is $\beta_{\mathrm{N}}(\epsilon)=3.12+3.5 \epsilon^{1.7}$. Using (a)

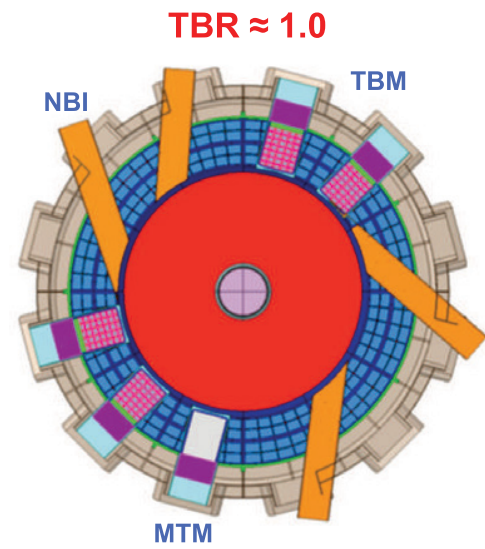

(b)

TBR $<1(\approx 0.9)$

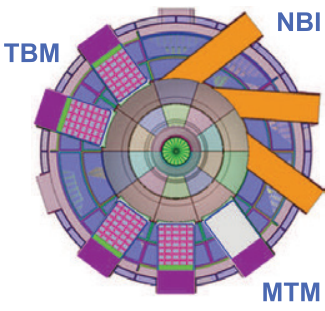

Figure 43. Side-by-side comparison of $R_{0}=1.7 \mathrm{~m}$ and $1.0 \mathrm{~m}$ ST-FNSF device sizes, TBR values, and port layouts. (a) $R=1.7 \mathrm{~m}$, $\mathrm{TBR} \approx 1.0$. (b) $R=1.0 \mathrm{~m}, \mathrm{TBR}<1(\approx 0.9)$.

maximum elongation data from NSTX at $A=1.45$ [207], $A=1.75$ [27], DIII-D record $\beta_{\text {T }}$ plasmas at $A=2.9$ and high $\kappa_{x}$ [208], and projections for the $A=4$ ARIES-AT power plant [122], a reasonable fit to the maximum achievable stable $\kappa_{x-\max }(\epsilon)=1.9+1.9 \epsilon^{1.4}$. These modeling/experimental data points and corresponding fits are shown in figure 45. In the calculations that follow, $\kappa_{x}(\epsilon)$ is reduced by $5 \%$ relative to the maximum value to provide additional stability margin, i.e. $\kappa_{x}(\epsilon)=0.95 \times \kappa_{x-\max }(\epsilon)$. More conservative scalings have also been proposed for tokamak DEMOs [209] with $\kappa_{x}(\epsilon)$ approximately 0.5 lower than the scaling proposed here, but the overall trend of decreasing $\kappa$ with increasing $A$ is otherwise similar.

The maximum achievable toroidal field $B_{\mathrm{T}}$ at the plasma geometric center $R_{0}$ is set by stress/strain limits in the TF magnet and HTS winding pack. The peak vertical stress in the inboard midplane of the TF magnet is the leading order stress, and if the inboard and outboard TF leg radii and $R_{0}$ are known, a simplified coil Lorentz force model [210] can be used to estimate the peak TF tensile stress. Then, given various assumed radial thicknesses for inboard SOL, first-wall, shield/ blanket, vacuum vessel, ohmic $(\mathrm{OH})$ solenoid, and clearances, 


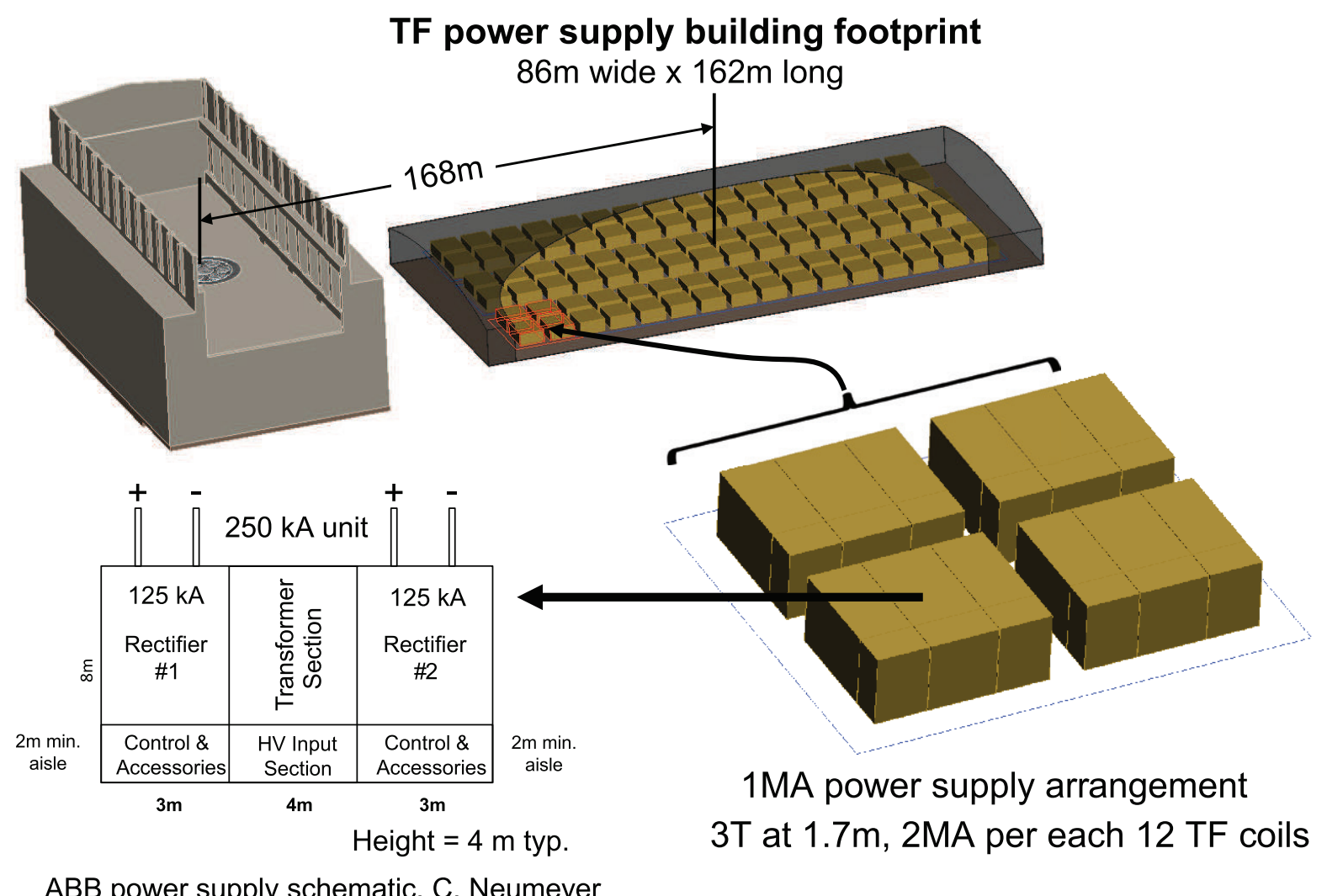

Figure 44. TF power supply building footprint for the $R_{0}=1.7 \mathrm{~m} \mathrm{ST-FNSF}$ device using conventional rectifier power supplies (figure reproduced with permission [170]).

the remaining radial build and cross-sectional area for TF coil winding pack and support structure can be calculated. Then, for a maximum allowable TF structural support stress of 0.66 GPa typical of stainless steel, winding pack current density (70 $\mathrm{MA} \mathrm{m}^{-2}$ ), and winding pack stress limited to $0.4 \mathrm{GPa}$ to ensure strains $\leqslant 0.3 \%$ to avoid any stress-related degradation in critical current [211], the maximum TF current and toroidal field can be computed.

A remaining critical parameter for determining fusion performance versus aspect ratio is the inboard shielding thickness and related radiation damage threshold for significant loss of critical current $I_{\mathrm{c}}$ or temperature $T_{\mathrm{c}}$. Recent studies for YBCO HTS tapes [212] show the fast neutron fluence $(E>0.1 \mathrm{MeV})$ threshold for serious $I_{\mathrm{c}}$ degradation (primarily in the $\mathrm{H} \| \mathrm{ab}$ plane) for $T_{\text {HTS }}<40 \mathrm{~K}$ is around $3.5 \times 10^{22} \mathrm{~nm}^{-2}$. When combined with water coolant, tungsten carbide (WC) is found to be the most effective neutron shield for superconducting magnets [40, 203, 205], but such shielding negatively impacts the breeding of the outboard DCLL blanket in ST devices [213].

Neutronics calculations assuming a water-cooled WC shield (10-15\% water by volume) show that approximately $60 \mathrm{~cm}$ of inboard shielding is needed to provide FNSF mission-relevant peak neutron fluences of 5-6 $\mathrm{MWy} \mathrm{m}^{-2}$ at the outboard midplane while staying below the inboard HTS TF magnet damage limit. Figure 46 shows a simplified model of an $A=2, R_{0}=1.87 \mathrm{~m}, P_{\text {fusion }}=550 \mathrm{MW} \mathrm{ST}$ plasma (grey) with a Gaussian D-T fusion source distribution (peaking at the plasma geometric center) surrounded by inboard shielding and outboard breeding blankets used to compute fast neutron

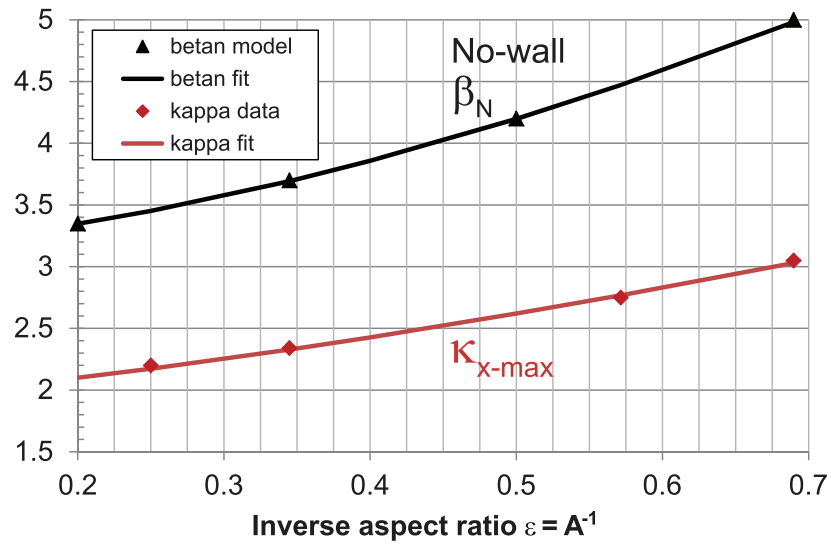

Figure 45. No-wall $n=1$ kink stability $\beta_{\mathrm{N}}$ limit and maximum double-null separatrix elongation $\kappa_{x}$ versus inverse aspect ratio.

attenuation through a WC shield and incident on a center column containing an HTS TF magnet. The model's nonplasma regions are shown in figure $46(a)$ and are assumed to be homogeneous in composition. The center column (green) composition is $57 \%$ copper, $38 \%$ steel, and $5 \%$ helium. The inboard shield (blue) is $87 \%$ WC and $13 \%$ water, the outboard first-wall (black arc) composition is $90 \%$ steel, $5 \%$ chromium zirconium copper, and 5\% helium, and the outboard shield (purple) is $70 \%$ EUROFER and $30 \%$ water. Finally, the outboard blanket (red) is assumed to be a solid breeder of $15 \%$ lithium orthosilicate, $55 \%$ beryllium multiplier, $20 \%$ helium, and $10 \%$ steel. The type of outboard breeding blanket (solid versus liquid) is not expected to significantly impact the 
(a)
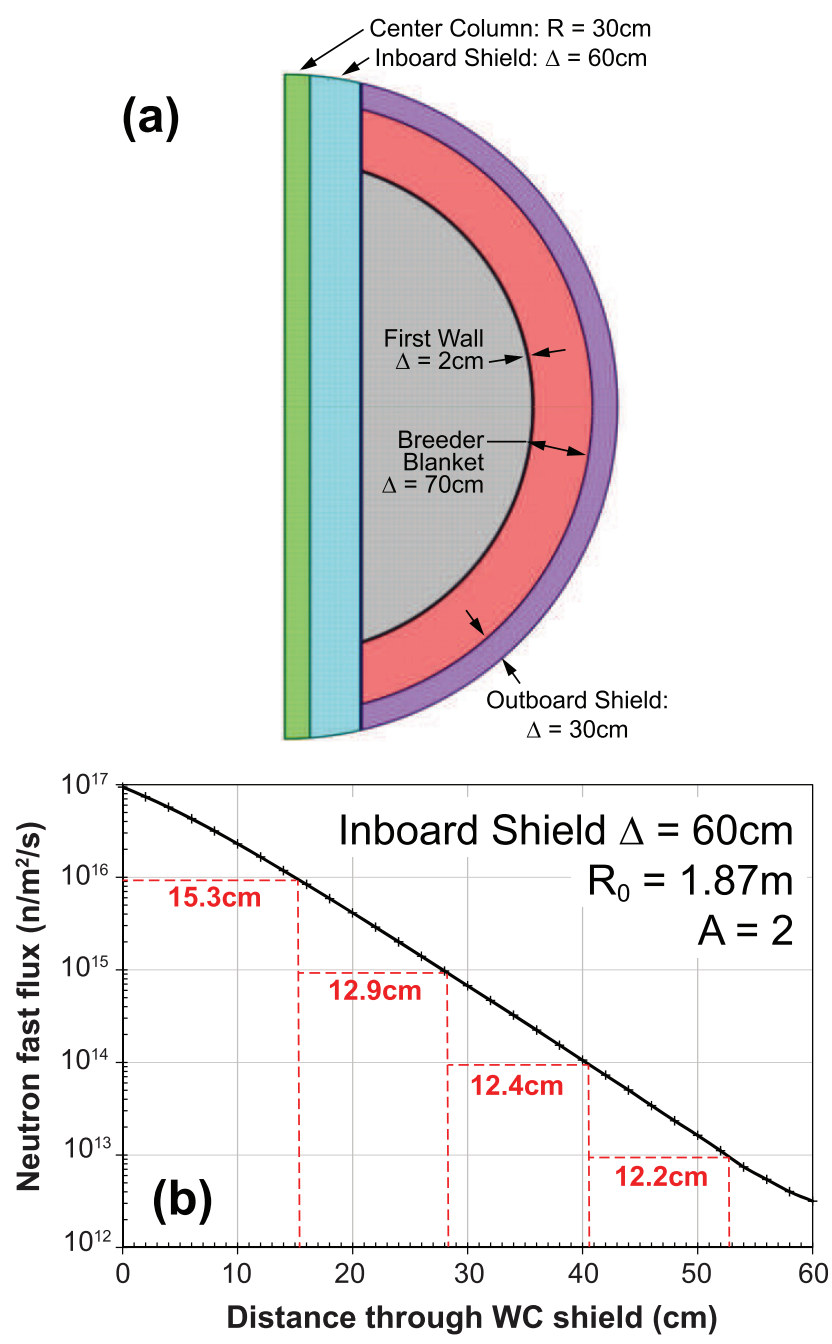

Figure 46. (a) Simplified model for computing fast neutron flux onto HTS TF magnet in center column, (b) fast neutron flux in inboard shield at vertical midplane as a function of distance from front of shield.

inboard shielding requirements. As shown in figure 46(b) for a $60 \mathrm{~cm}$ inboard shield thickness, the first order of magnitude of fast neutron flux attenuation has a decay length of $15 \mathrm{~cm}$, subsequent decadal decay lengths decrease from 13 to $12 \mathrm{~cm}$, and the effective decay length per decade for the full $60 \mathrm{~cm}$ shield is $13.4 \mathrm{~cm}$. More detailed calculations including ferritic steel structure (which has lower neutron attenuation) to support and contain the WC and water coolant indicate longer shield-averaged decay lengths of $15-16 \mathrm{~cm}$ per decade.

As shown in section 4.2, no or only small inboard breeding may be required to achieve $\mathrm{TBR} \approx 1$ if the aspect ratio is sufficiently low $(A=1.7-1.8)$, the major radius is sufficiently high $\left(R_{0} \gtrsim 1.7 \mathrm{~m}\right)$, and long-leg divertors are used to enable breeding at the top and bottom of the central column. Neutronics studies indicate that if the aspect ratio is increased to $A=2$, some inboard breeding ( $10 \mathrm{~cm}$ thick DCLL covering most of the high-field/inboard side) is needed to achieve $\mathrm{TBR} \approx 1$ assuming a $1 \mathrm{~m}$ thick conformal outboard DCLL breeding blanket. Previous calculations indicated that for $A=4$ AT pilot plants [3] $40 \mathrm{~cm}$ of inboard breeding blanket, $40-50 \mathrm{~cm}$ of inboard shielding, and $80 \mathrm{~cm}$ of outboard breeding
Shield and blanket thickness [m]

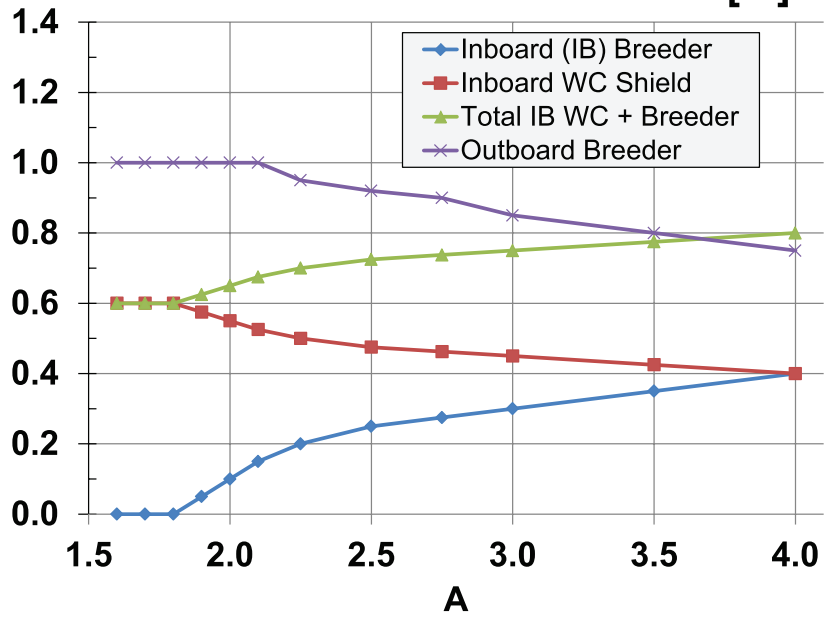

Figure 47. Simplified model for WC shield and breeding blanket thickness versus aspect ratio.

blanket are sufficient for TBR $\approx 1$. From these results it can be concluded that inboard breeding blankets must be roughly twice as thick as WC shielding to achieve the same shielding effectiveness. With these parameters it is possible to construct an approximate shielding and blanket thickness scaling versus aspect ratio that achieves TBR $\approx 1$ and shields the HFS HTS TF magnet sufficiently well to access peak outboard neutron fluences of 5-6 $\mathrm{MWy} \mathrm{m}^{-2}$. The thicknesses for this approximate scaling are shown in figure 47 . The net result is that thicker inboard blankets and overall shield + blanket thicknesses are needed at higher $A$ to achieve TBR $\approx 1$ at the same effective shielding as $60 \mathrm{~cm}$ of WC. More complete 3D calculations of TBR are planned to accurately compute shielding and blanket thickness requirements versus aspect ratio, but figure 47 should capture the leading order trends.

\subsection{Fusion performance versus aspect ratio}

With the baseline shielding and breeding requirements chosen as shown in figure 47, scaling studies find that a plasma major radius of $R_{0}=3 \mathrm{~m}$ can achieve both peak neutron fluences of at least 5-6 $\mathrm{MWy} \mathrm{m}^{-2}$ and also $Q_{\mathrm{eng}} \geqslant 1$ for a wide range of aspect ratios and confinement assumptions. This finding motivates the choice of fixed plasma major radius $R_{0}=3 \mathrm{~m}$ for all HTS FNSF/pilot plant calculations that follow. For these scaling studies the Greenwald fraction is again chosen to be $0.8,0.5 \mathrm{MeV}$ NNBI is assumed for heating and CD, and $P_{\mathrm{NBI}}$ is fixed at $50 \mathrm{MW}$ unless otherwise noted. $H_{98}$ is adjusted to achieve full non-inductive current (bootstrap $+\mathrm{NBI}$ ) and hold the total $\beta_{\mathrm{N}}(\epsilon)$ fixed as shown in figure 45 . The $q^{*}$ value is not constrained but is typically above 3 . The same power production assumptions from section 2.1.6 and equation (1) are used but with $\eta_{\text {th }}=0.45$ and $\eta_{\text {aux }}=0.3$ (instead of 0.4 ).

Figure 48 shows the magnetic field resulting from the scans of aspect ratio $A$ and effective (i.e. relative to watercooled WC) inboard shielding thickness $\Delta_{\text {sh-eff. The refer- }}$ ence scenario has $\Delta_{\mathrm{sh}-\text { eff }}=60 \mathrm{~cm}$, and for these scans, the thickness of the inboard shield and any inboard blanket are assumed to scale linearly together so all aspect ratios have the 
$B_{T}$ at geometric center [T]
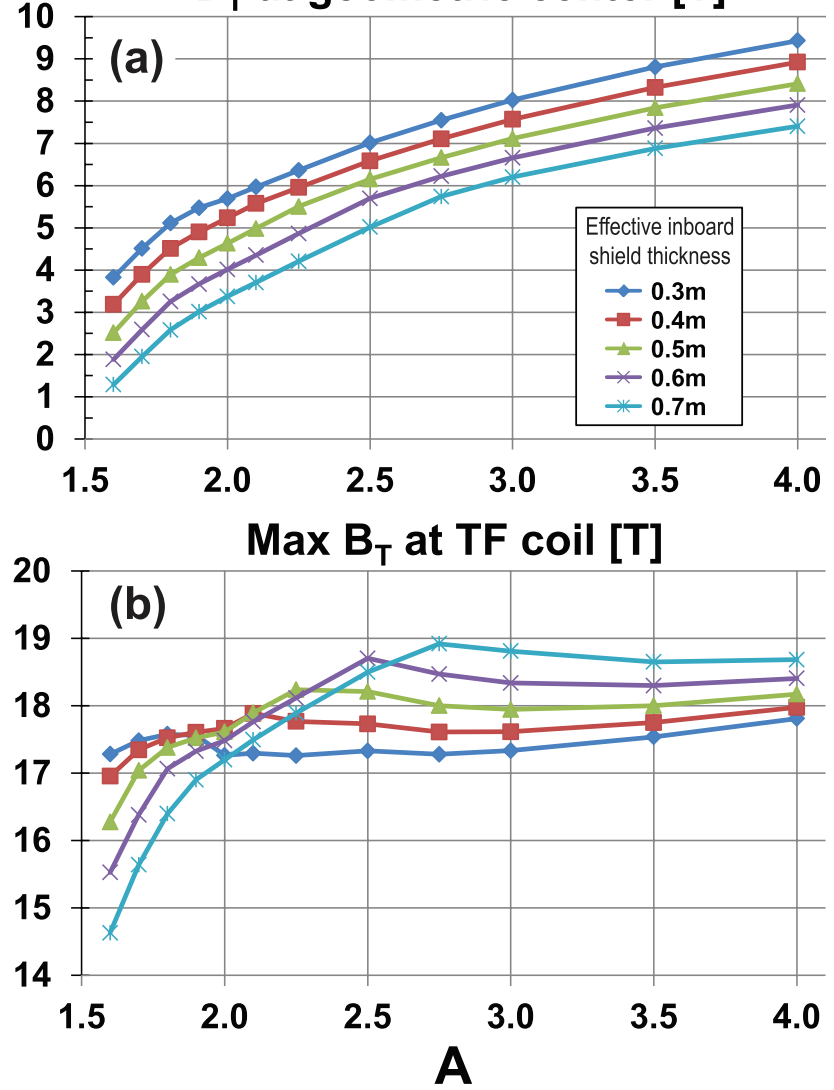

Figure 48. (a) Vacuum toroidal magnetic field at plasma geometric center, and $(b)$ peak fields at TF magnet for $R_{0}=3 \mathrm{~m}$ HTS TF ST/AT pilot plants versus aspect ratio $A$ and effective (WC-equivalent) inboard shielding thickness.

same $\Delta_{\text {sh-eff. This also implies any aspect ratios that require }}$ inboard breeding will have TBR $<1$ when $\Delta_{\text {sh-eff }}<60 \mathrm{~cm}$. The range $\Delta_{\text {sh-eff }}=30-70 \mathrm{~cm}$ is chosen to scan from very thin shields where refrigerator power limits due to nuclear heating become important even at elevated magnet temperatures $T=20-40 \mathrm{~K}$ [205] to relatively thick shields that should provide magnet lifetimes several times longer than needed for achieving FNSF-relevant neutron fluences.

Figure 48(a) shows that for the thinnest effective shields $(30 \mathrm{~cm})$ the vacuum toroidal field $B_{\mathrm{T}}$ at the geometric center $R_{0}=3 \mathrm{~m}$ increases from 4 to $9 \mathrm{~T}$ as the aspect ratio $A$ is varied from $A=1.6$ to 4 . Each $10 \mathrm{~cm}$ increment in shielding reduces $B_{\mathrm{T}}$ by $0.7 \mathrm{~T}$ at the lowest aspect ratios and by $0.45-0.5 \mathrm{~T}$ at the highest aspect ratios. However, as expected, the relative reduction in field is much larger at low-A. For example, increasing the shield thickness from $30 \mathrm{~cm}$ to $60 \mathrm{~cm}$ reduces the $A=1.6$ field by a factor of 2 but reduces the field in the plasma by only $16 \%$ at $A=4$. Figure $48(b)$ shows that the peak field at the TF magnet is between $17 \mathrm{~T}$ and $19 \mathrm{~T}$ for nearly all configurations except for the lowest-A cases with thicker shields. Clearly the ability of HTS to remain superconducting at high field is critical to taking advantage of the higher winding-pack current density assumed for these configurations.

Figure 49 plots the fusion and electricity gains and fusion and net electrical powers for the aspect ratio and shielding thickness scans. Figure 49(a) shows that for thinner shields
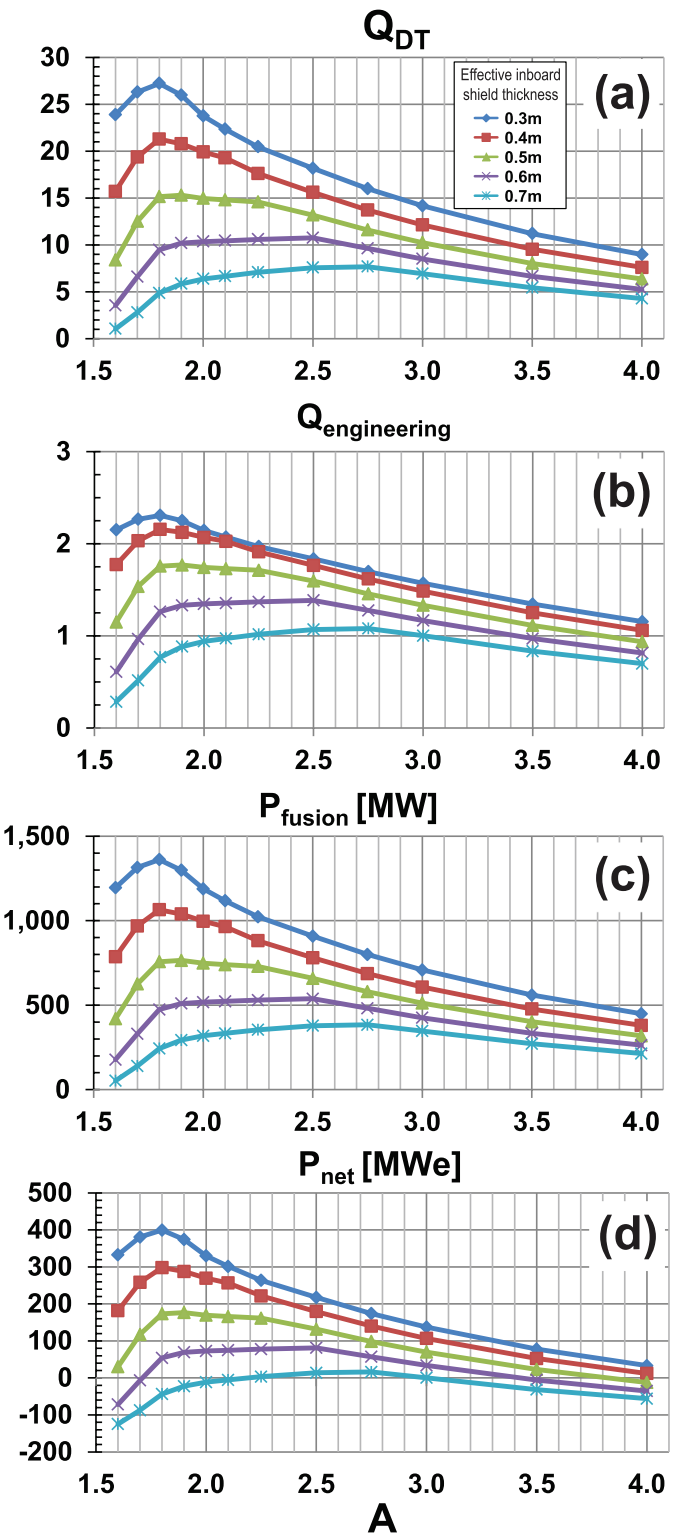

Figure 49. (a) Fusion gain $Q_{\mathrm{DT}},(b)$ engineering gain $Q_{\mathrm{eng}},(c)$ fusion power $P_{\text {fusion }}$, and $(d)$ net electrical power for $R_{0}=3 \mathrm{~m}$ HTS ST/AT pilot plants versus aspect ratio $A$ and effective inboard shielding thickness.

the fusion gain is maximized near $A=1.8$ with $Q_{\mathrm{DT}}>20$. The fusion gain is nearly independent of $A$ between $A=1.9$ and 2.5 for $\Delta_{\text {sh-eff }}=60 \mathrm{~cm}$ and is maximized near $A=2.5-2.7$ for $\Delta_{\mathrm{sh}-\text { eff }}=70 \mathrm{~cm}$. As stated previously, for these scans the auxiliary power is fixed at $P_{\mathrm{NBI}}=50 \mathrm{MW}$ and the confinement is adjusted to operate at the nominal no-wall $\beta_{\mathrm{N}}$ limit shown in figure 45 . These results are consistent with the finding that $A<2$ scenarios have the potential for high fusion gain for thin inboard shielding [214] provided the shield is thick enough to have acceptable refrigeration power for the HTS TF magnet [205]. Figure 49(b) shows that all aspect ratios can make net electricity for the thinnest shield cases, $Q_{\text {eng }}$ is nearly independent of $A$ (at $Q_{\mathrm{eng}} \approx 1.4$ ) between $A=1.9$ and 2.5 for $\Delta_{\text {sh-eff }}=60 \mathrm{~cm}$, and $Q_{\text {eng }} \gtrsim 1$ for $A=2$ to 3 for $\Delta_{\text {sh-eff }}=70 \mathrm{~cm}$. Figures $49(c)$ and $(d)$ show that for 
the reference $\Delta_{\text {sh-eff }}=60 \mathrm{~cm}$ configuration and between $A=1.9$ and 2.5 , the fusion power $P_{\text {fusion }} \approx 550 \mathrm{MW}$ and the net electric power $P_{\text {net }}=90-100 \mathrm{MW}$.

Figure 50 plots several dimensionless parameters for the aspect ratio and shielding thickness scans. Figure 50(a) shows that the required $H_{98}$ is very nearly independent of shield thickness except for the lowest-A and thickest shields cases. For all cases, the $H_{98}$ required to operate fully non-inductively and at the no-wall limit is well above 1 , i.e. $H_{98} \approx 1.75-1.8$ for $A \leqslant 2$ and decreases to $\approx 1.55$ at $A=4$. Interestingly, using a confinement scaling by Petty [73] for which turbulent transport is assumed to be dominated by electrostatic turbulence and therefore having no/weak $\beta$ degradation [214] (unlike the ITER ELMy H-mode scaling which varies as $\sim \beta^{-0.9}$ ), there is a more substantial variation in the required $H_{\text {Petty-08 }}$ as a function of shield thickness. Overall, the required $H_{\text {Petty }-08}$ values are systematically lower than the required $H_{98}$ values, and for the reference $\Delta_{\text {sh-eff }}=60 \mathrm{~cm}$ configuration $H_{\text {Petty-08 }} \approx 1.3$ nearly independent of aspect ratio. As discused in section 2.1.4, the scaling of confinement with dimensionless parameters remains an important research topic for tokamaks of all aspect ratios. Figure $50(b)$ shows that the bootstrap fraction $f_{\mathrm{BS}}$ is between $70-81 \%$ for nearly all scenarios treated and weakly dependent on aspect ratio. Figure 50(c) shows $\beta_{\mathrm{T}} \approx 3.5 \%$ at $A=4,7-10 \%$ at $A=2$, and $10-20 \%$ at $A=1.6$ depending on shield thickness. Lastly, figure 50 $(d)$ shows that $q^{*}>3$ for all cases studied with the highest values $q^{*} \geqslant 4$ occurring between $A=1.8$ and 2.25 even for the thickest shielding cases.

As discussed in section 2.4.3, non-solenoidal plasma current formation and ramp-up are significant challenges for ST-based FNSF and pilot plant devices. An important question is what is the minimum aspect ratio for possible inclusion of a central solenoid for current formation and ramp-up. Figure 51(a) shows the steady-state plasma current varies strongly with aspect ratio and approximately as $I_{\mathrm{P}} \propto A^{-1}$ except for the lowest-A and thicker shield cases. The required currents at the lowest $A=1.6$ and thinnest shield are also relatively high $=15-18 \mathrm{MA}$ and exceed ITER levels. As the aspect ratio is increased, there is increasing space for an ohmic heating $(\mathrm{OH}) /$ central solenoid coil for plasma initiation and possibly ramp-up to the steady-state plasma current value. For the aspect ratio scans treated here, the TF winding pack thickness is held constant at $24 \mathrm{~cm}$, and TF external structure thickness is held constant at $20 \mathrm{~cm}$ from $A=1.6$ to 2 , and is increased approximately linearly with A to $45 \mathrm{~cm}$ at $A=4$. This aspect ratio dependence is chosen to keep the overall inboard midplane TF magnet tensile stress $\lesssim 0.5-0.55 \mathrm{GPa}$. The remaining space inboard of the TF magnet structure is allocated to a central solenoid with HTS conductor current density of $70 \mathrm{MA} \mathrm{m}^{-2}$ and sized to have a maximum internal vertical field of $20 \mathrm{~T}$. The $\mathrm{OH}$ flux swing can be related to the plasma current via an Ejima-Wesley coefficient $C_{\mathrm{E}-\mathrm{W}}(A)$ as $\Delta \Psi_{O H}=C_{\mathrm{E}-\mathrm{W}}(A) \mu_{0} R_{0} I_{\mathrm{P}}$ where $C_{\mathrm{E}-\mathrm{W}}(A)=0.18 \times A$ is linear in aspect ratio [215] but has a reduced coefficient $(0.18$ versus 0.4 ) consistent with auxiliary-heated H-mode ramp-up as observed in NSTX [27]. Figure 51(b) shows that substantial ( $>50 \%$ ) single-swing current ramp-up can be achieved for the thinnest shield case for $A \geqslant 2$ but this fraction is only
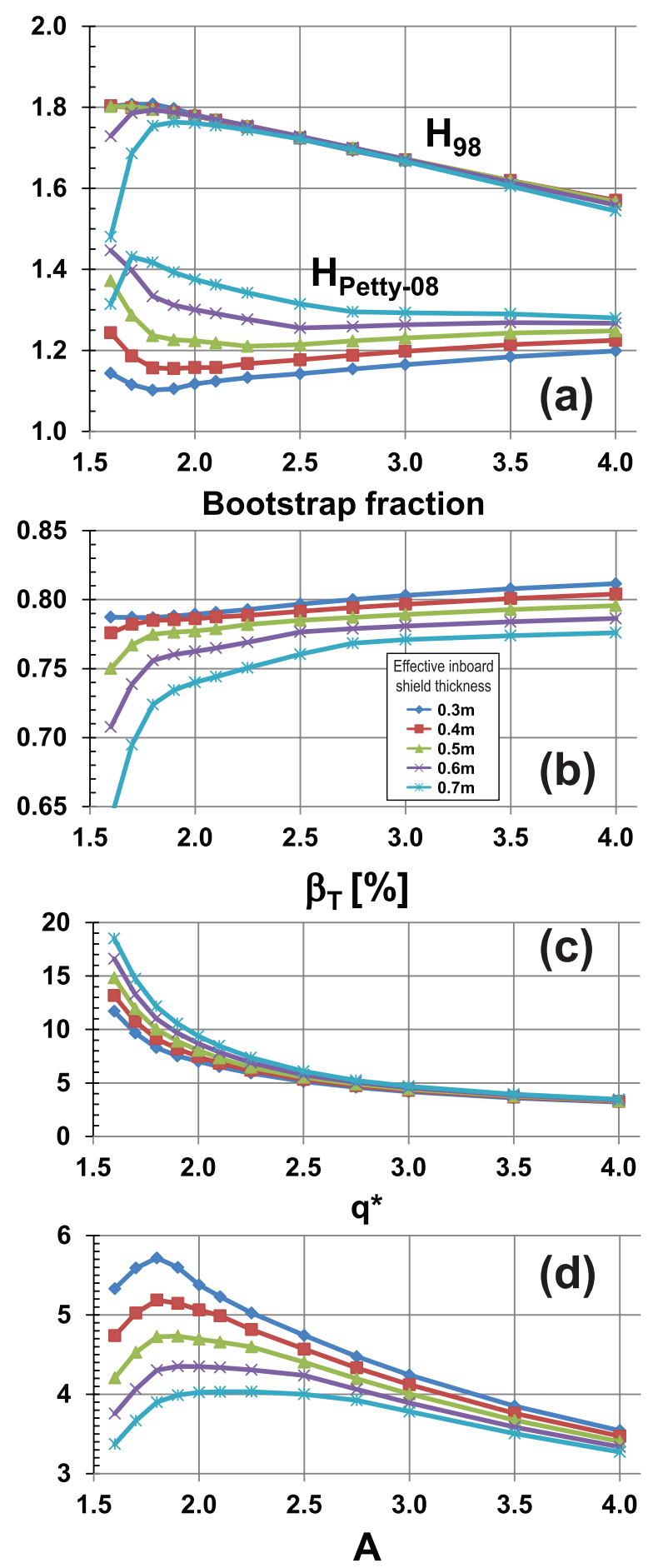

Figure 50. (a) Confinement multipliers $H_{98}$ and $H_{\text {Petty-08, }}(b)$ bootstrap fraction, $(c)$ toroidal beta, and $(d)$ kink safety factor $q^{*}$ for $R_{0}=3 \mathrm{~m}$ HTS ST/AT pilot plants versus aspect ratio $A$ and effective inboard shielding thickness.

achievable for $A \geqslant 3$ for the thickest shield case. If instead one considers only the minimum current value $\approx 2$ MA needed to efficiently absorb NNBI (see section 2.4.3), and if doubleswing $\mathrm{OH}$ could be used (with a long solenoid current rampdown to minimize negative loop voltage), then figure 51(c) shows that $A \geqslant 2$ is required for the reference $\Delta_{\text {sh-eff }}=60 \mathrm{~cm}$ configuration. 

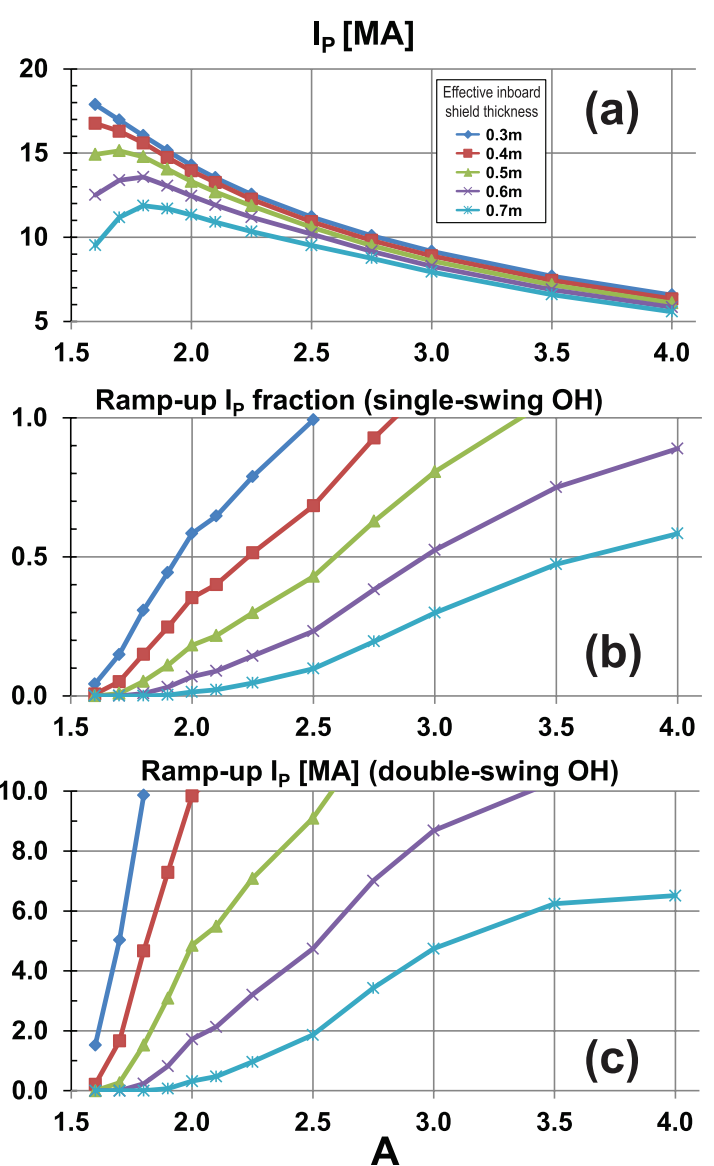

Figure 51. (a) Steady-state full-non-inductive plasma current, (b) fraction of steady-state plasma current achievable with assist of single-polarity swing of ohmic heating $(\mathrm{OH})$ solenoid, and (c) ramp-up plasma current achievable with double-swing $\mathrm{OH}$ for $R_{0}=3 \mathrm{~m}$ HTS ST/AT pilot plants versus aspect ratio $A$ and effective inboard shielding thickness.

In terms of wall loading and power handling, figure 52(a) shows the ratio of the total heating power $P_{\text {heat }}$ (NBI + alpha heating) normalized to the plasma surface area $S$, and this value is near $1 \mathrm{MW} \mathrm{m}^{-2}$ and nearly independent of aspect ratio for the thinnest shield cases. For the thicker shield cases $P_{\text {heat }} / S$ is highest for $A>2.5$. Figure 52(b) shows that the surface-average neutron wall loading is maximized for $A=1.8$ to 2.5 for thinner shields but is maximized between $A=2.5$ to 2.75 for thicker shield cases with the average wall loading at $A=2.5$ approximately $30 \%$ higher than for $A=2$ for the reference $\Delta_{\text {sh-eff }}=60 \mathrm{~cm}$ configuration. Projecting the cost of future FNSF/pilot plant devices is extremely challenging, but it is possible to compare relative masses or (assuming similar component mass densities) compare estimated volumes of core fusion components. Two of the more complex and/or costly objects in the fusion core are likely to be the toroidal field magnets and the blanket modules. The achievable $Q_{\text {eng }}$ is a useful metric for overall device performance. At fixed heating power $Q_{\text {eng }} \propto Q_{\text {DT }} \propto P_{\text {fusion. Figure 52(c) shows }}$ that the fusion power normalized to the blanket volume has a maximum value near $A=3$ for all shielding cases considered, and is nearly independent of $A$ for $A \geqslant 2.5$. For the reference $\Delta_{\mathrm{sh}-\text { eff }}=60 \mathrm{~cm}$ configuration, $A=2$ is found to require
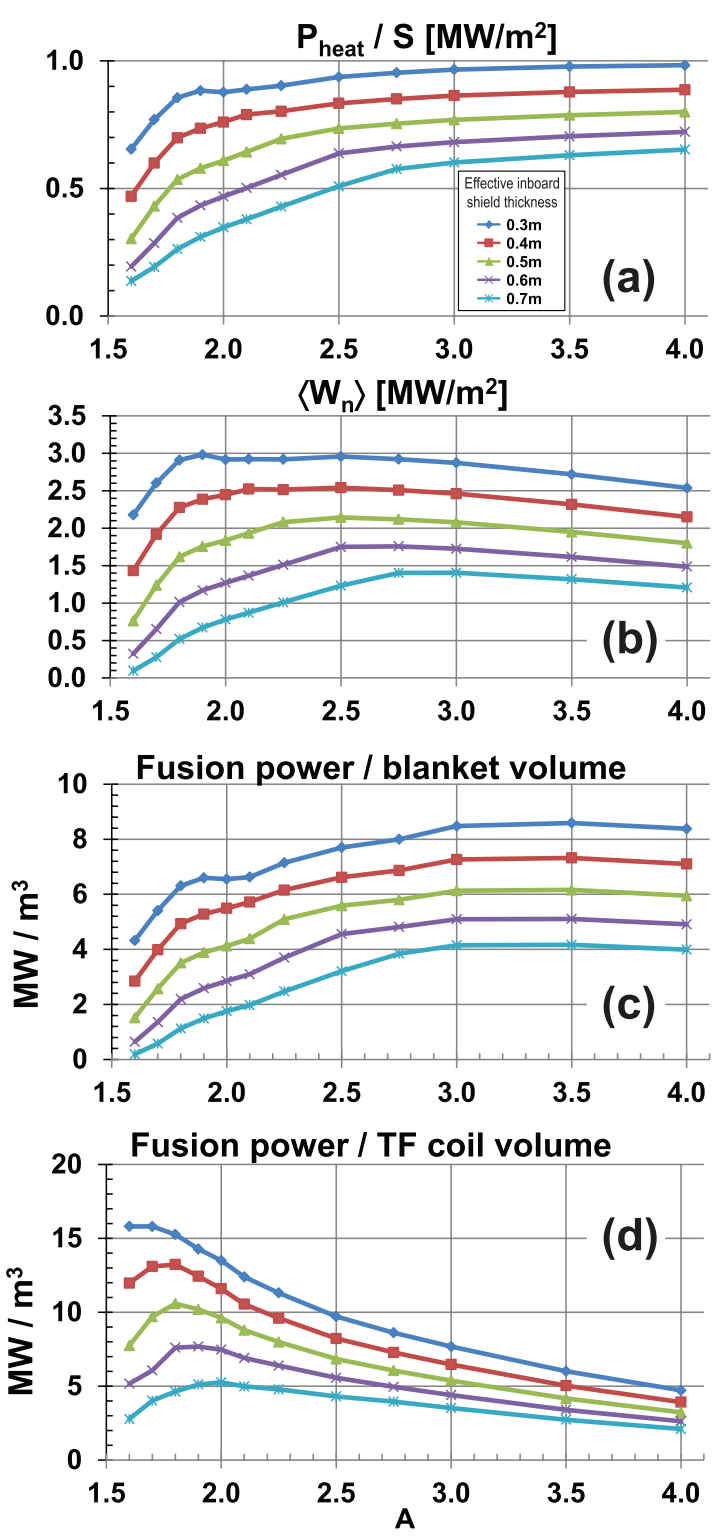

Figure 52. (a) Total heating power $(\mathrm{NBI}+\alpha)$ normalized to surface area at plasma boundary, $(b)$ surface-average neutron flux at plasma boundary, $(c)$ fusion power normalized to estimated volume of breeding blankets, and $(d)$ fusion power normalized to estimated volume of toroidal field magnets for $R_{0}=3 \mathrm{~m}$ HTS $\mathrm{ST} / \mathrm{AT}$ pilot plants versus aspect ratio $A$ and effective inboard shielding thickness.

60-70\% more blanket volume than $A=2.5$ or 3 at similar $Q_{\text {eng. }}$ On the other hand, figure $52(d)$ shows that $A=1.6$ to 2 maximizes the fusion power per unit TF coil volume for all shielding thicknesses, and lower-A would also obviously minimize $\mathrm{OH}$ coil volume. Thus, lower-A would likely minimize core TF and CS magnet cost while higher-A would likely minimize blanket cost for otherwise similar overall fusion performance.

The results of figure 52 imply that depending on the mission emphasis (for example shorter-duration $Q_{\mathrm{eng}}>1 \mathrm{dem}$ onstration versus high neutron fluence goal) different aspect ratios may be optimal. Figure 53 shows this fluence and HTS TF magnet lifetime trade-off versus shielding thickness. In this figure, the peak inboard/outboard neutron fluxes are taken to 


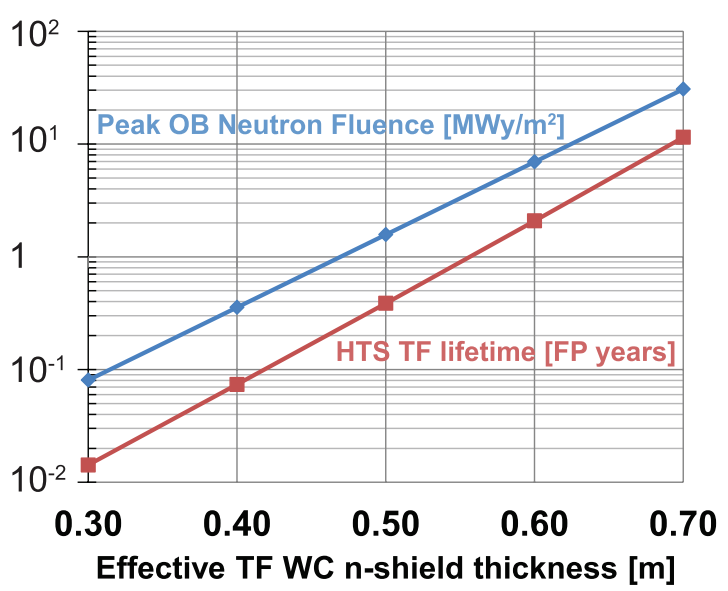

Figure 53. Minimum HTS TF magnet lifetime in full-power years selected from all aspect ratios (red) and peak outboard neutron fluence (blue) versus effective inboard shielding thickness for $R_{0}=3 \mathrm{~m}$ HTS ST/AT pilot plants.

be 1.4/1.9 times the surface-average fluxes, respectively. Note that in this figure the HTS magnet lifetime decreases faster with decreasing shield thickness than the fluence because the fusion power and wall loading increase as the shielding thickness decreases. These results imply that at fusion power levels of $\sim 500 \mathrm{MW}$ the magnet lifetime would be reduced to a few full-power weeks to months for 30 to $40 \mathrm{~cm}$ of equivalent WC shield thickness, respectively.

\subsection{Preliminary concept for an $A=2$ HTS-TF FNSF/pilot plant}

The high current density and high toroidal field potentially possible with HTS magnets combined with reduced aspect ratio to maximize the effectiveness of long-leg/super-X divertors in mitigating high heat fluxes while retaining TBR near 1 opens the possibility of all superconducting FNSF/pilot plants at $R_{0}=3$ m with $A \leqslant 2$. For $A=2$, a relatively high $H_{98} \approx 1.8$ $\left(H_{\text {Petty-08 }}=1.1-1.35\right)$ is required to reach the no-wall limit as shown in figure 50. Figure 54(a) shows that this requirement can be relaxed by increasing the NBI heating power and reducing $Q_{\text {eng }}$ from 1.4 to 1 for the reference $\Delta_{\text {sh-eff }}=60 \mathrm{~cm}$ configuration. As seen in the figure, increasing $P_{\mathrm{NBI}}$ to $100 \mathrm{MW}$ reduces the required $H_{98}$ to 1.4 (values already achieved on NSTX) and the required $H_{\text {Petty }-08}$ is reduced to 1 . Figure 54(b) shows that for these reduced $Q_{\mathrm{eng}}=1$ scenarios the $\beta_{\mathrm{N}}$ is at or below 4 and the average neutron wall loading could be as high as $1.7 \mathrm{MW} \mathrm{m}^{-2}$ (3.2 $\mathrm{MW} \mathrm{m}^{-2}$ outboard-peak) for $100 \mathrm{MW}$ of NBI heating power.

In the interest of scoping this low-A configuration while also retaining a small solenoid for plasma start-up, an $A=2$ FNSF/pilot plant concept is considered with $60 \mathrm{~cm}$ effective inboard shielding thickness, $B_{\mathrm{T}}=4.1 \mathrm{~T}, \kappa_{x}=2.5, \beta_{\mathrm{N}}=4.2$, $P_{\mathrm{NBI}}=50 \mathrm{MW}$, and $P_{\text {fusion }}=560 \mathrm{MW}$, i.e. parameters all consistent with the results shown in figures 48-54. Building on the configuration ideas developed for the $\mathrm{Cu}-\mathrm{TF}$ ST-FNSF and also previous low-A SC DEMO reactor designs [197, 216, 217] but incorporating long-leg outboard divertors and a vertical maintenance strategy, figure 55 shows sectional

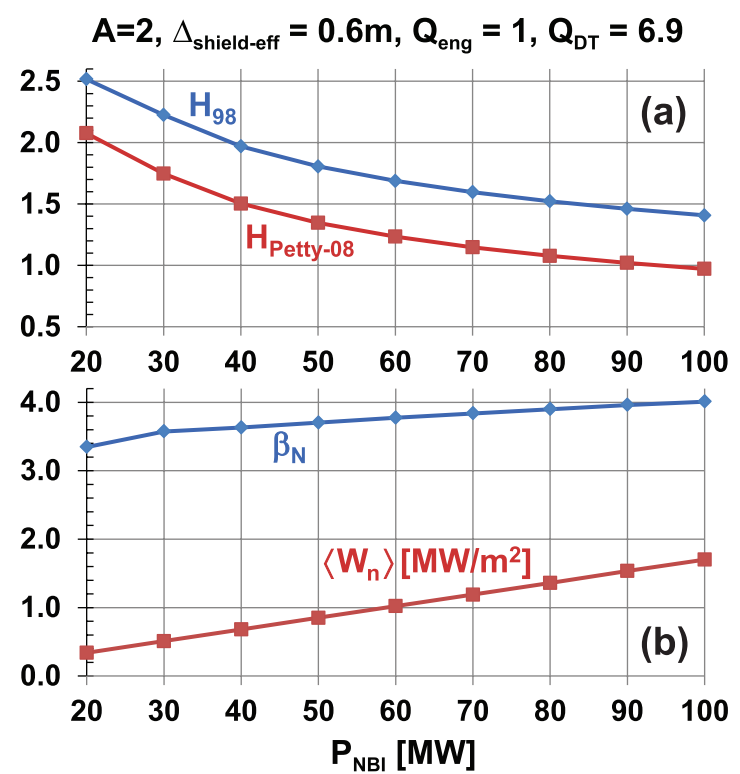

Figure 54. (a) Confinement multipliers $H_{98}$ and $H_{\text {Petty }-08}$ and $(b)$ normalized beta and surface-average neutron flux at plasma boundary at $A=2$ with an effective inboard shielding thickness $=0.6 \mathrm{~m}$ and fixed $Q_{\mathrm{eng}}=1$ and $Q_{\mathrm{DT}}=6.9$.

views of an $R_{0}=3 \mathrm{~m}, A=2 \mathrm{HTS}-\mathrm{TF}$ FNSF/pilot plant concept. Figure 55 shows that it is possible to have a configuration that incorporates [170]:

1. Continuous HTS TF coils with no joints

2. All equilibrium PF coils are superconducting and outside the TF (and therefore just as well shielded as the TF magnet)

3. Inboard divertor PF coils to support equilibria with $\kappa_{x}=2.5$ and $\delta_{x}=0.5$

4. Top/bottom PF coils to support a long-leg/super-X divertor configuration

5. Space for a small HTS solenoid for plasma current initiation up to $2 \mathrm{MA}$

6. Integrated outboard blanket + shield + divertor modules

For this configuration the outboard blanket + shield + divertor modules are toroidally segmented and compatible with a vertical maintenance strategy where modules are removed through ports between the TF coils. To keep most of the cold mass at cryogenic temperatures during maintenance, the upper/top two PF coils are located in a separate cryo-stat that is removed with the larger upper cryostat lid during vertical maintenance activities. Finally, using the same power exhaust scaling assumptions as in section 2.3 and figures $19(a)$ and (b), figure 56 shows that it is possible to have peak divertor heat-fluxes below $10 \mathrm{MW} \mathrm{m}^{-2}$ with the strike-point on either the upper or lower target plate in the divertor slot. Divertor scenarios with the strike-point as shown in figure 56(b) combined with upstream pumping could be favorable for stable full detachment [152]. For both configurations all equilibrium PF coils have SC winding pack current densities below 40 $\mathrm{MA} \mathrm{m}^{-2}$. These results show that if TF and OH HTS winding pack current densities $\geqslant 70 \mathrm{MA} \mathrm{m}^{-2}$ and peak fields up to $20 \mathrm{~T}$ could be achieved, and non-inductive current ramp-up reliably 


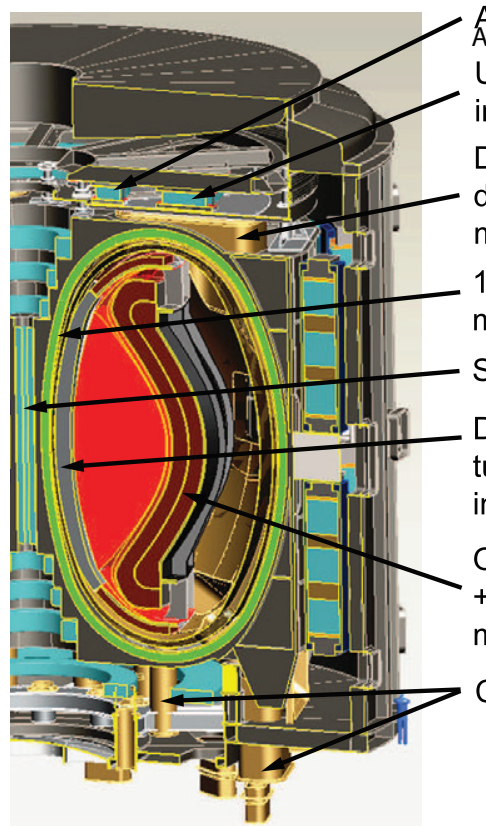

All SC PF coils outside TF

Upper SC PF coils located

in a separate cryostat

Device configuration designed for vertical maintenance

10 continous HTS TF magnets (no joints)

Small HTS solenoid

Double-wall vessel + tungsten carbide shield + inboard blanket

Outboard blanket + shield

divertor sized for vertical maintenance scheme

Coolant supply at bottom

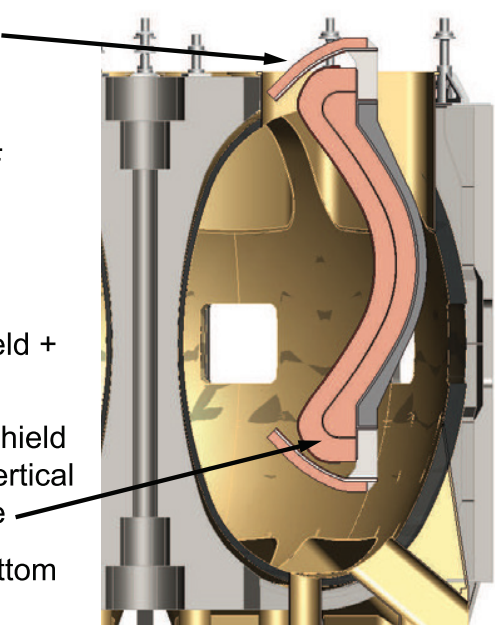

Figure 55. Cross-sectional views of $A=2, R_{0}=3 \mathrm{~m} \mathrm{HTS} \mathrm{FNSF/pilot} \mathrm{plant.}$
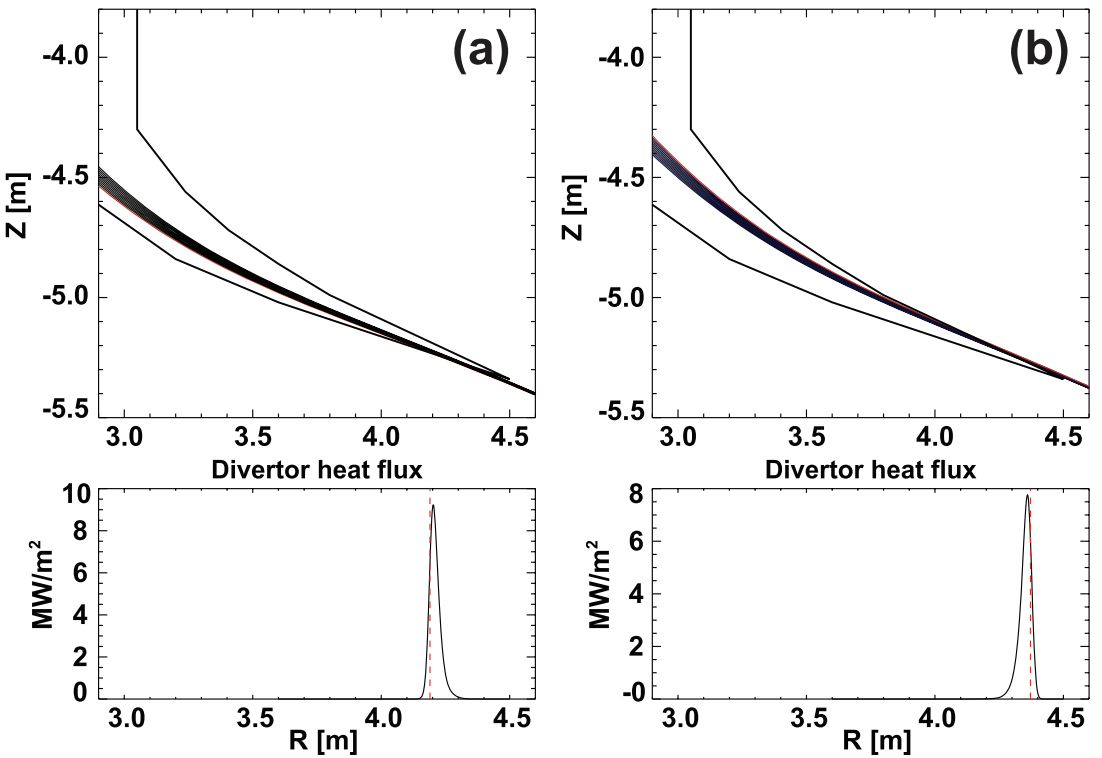

Figure 56. (Upper) Long-leg divertor regions including limiter outlines (thicker black lines) and poloidal flux contours (thinner black lines) with separatrix flux contours shown in red, and (bottom) divertor heat flux profiles with radii of strike-points indicated by red dashed line for divertor target locations ( $a$ ) farther and $(b)$ closer to mid-plane for $A=2, R_{0}=3 \mathrm{~m} \mathrm{HTS} \mathrm{FNSF} /$ pilot plant with $P_{\mathrm{NBI}}=50 \mathrm{MW}$ and $P_{\text {fusion }}=560 \mathrm{MW}$

demonstrated in near-term ST experiments, an $R_{0}=3 \mathrm{~m}, A=2$ superconducting $\mathrm{FNSF} /$ pilot plant capable of $6 \mathrm{MWy} \mathrm{m}^{-2}$ (peak), $Q_{\text {eng }}>1, \mathrm{TBR} \approx 1$, and having significantly reduced TF magnet volume may be feasible. For a net electricity mission, an HTS TF device would be more conservative from a physics and blanket and auxiliary system technology standpoint than the corresponding $\mathrm{Cu}$ TF device at $R_{0} \geqslant 1.75$ and $H_{98}=1.5$ (see figures 12 and 54 for comparison). In particular, the low-A HTS device can achieve electricity breakeven at higher $q^{*}$ (4-4.5 versus 3$)$, lower $\beta_{\mathrm{N}}$ (4 versus 5.5), lower $\eta_{\text {th }}(0.45$ versus 0.59$)$, lower NBI wall-plug efficiency (0.3 versus 0.4$)$, and could utilize a small solenoid for plasma current initiation. Further, even with these more conservative parameters, the HTS TF device has a higher $Q_{\text {eng }}$ (3.7 versus 2.8 [3]) when extrapolated to $1 \mathrm{GW}$ electric by increasing the device major radius to $4.5-5 \mathrm{~m} / 3.2 \mathrm{~m}$ for the HTS/Cu TF devices, respectively.

\section{Summary}

A fusion nuclear science facility (FNSF) could play an important role in the development of fusion energy by providing the nuclear environment needed to develop fusion materials and components. For the first time, copper-TF ST-based FNSF 
configurations have been developed simultaneously incorporating several important features including: (1) a blanket system capable of tritium breeding ratio $\mathrm{TBR} \approx 1$, (2) a poloidal field coil set supporting high elongation and triangularity for a range of internal inductance and normalized beta values, (3) a long-legged/super-X divertor which substantially reduces projected peak divertor heat-flux and has all outboard poloidal field coils outside the vacuum chamber and as superconducting to reduce power consumption, and (4) a vertical maintenance scheme in which blanket structures and the centerstack can be removed independently. Negative neutral beam injection (NNBI) heating and current drive is calculated to effectively support full non-inductive operation including non-inductive current overdrive ramp-up starting from initial plasma current levels as low as 2 MA. The NNBI blanket penetrations do reduce the TBR, but if NBI power fluxes as high as $50 \mathrm{MW} \mathrm{m}^{-2}$ through blanket apertures can be supported, $80 \mathrm{MW}$ of NNBI heat would reduce the TBR in a $R_{0}=1.7 \mathrm{~m}$ device by only $3 \%$. Tangential injection and breeding at the back of the blanket are both computed to help reduce the impact of the NBI penetrations on TBR.

A long-leg divertor also plays a very important role by moving the divertor strike-point to radii locally outboard of the conformal blanket modules. This location not only provides some shielding of the divertor target from neutron fluxes, but also frees the inboard top and bottom regions of the device for tritium breeding. For example, for a $R_{0}=1.7 \mathrm{~m}$ ST-FNSF device, the top/bottom breeding can increase the total TBR by at least $7 \%$. Overall, at $A=1.7$, the plasma geometric major radius threshold for tritium self-sufficiency in a $\mathrm{Cu}$-TF ST-FNSF is found to be approximately $1.7 \mathrm{~m}$. A smaller $R_{0}=1 \mathrm{~m}$ ST-FNSF device has TBR $\approx 0.9$ which is below unity but substantially reduces $\mathrm{T}$ consumption relative to not breeding. For a $R_{0}=1 \mathrm{~m}$ device it would be necessary to purchase approximately $0.4-0.55 \mathrm{~kg}$ of T/FPY from outside sources at a cost of $30-200 \mathrm{~kg}^{-1}$ of $\mathrm{T}$ implying a total cost of $12-110 \mathrm{M}$ per full-power year. Shielding calculations indicate that the vacuum vessel, TF coils, outboard PF coils, and most or all of the divertor PF coils can be lifetime components for both $\mathrm{Cu}$-TF ST-FNSF devices, i.e. could support the neutron fluence mission of $6 \mathrm{MWy} \mathrm{m}^{-2}$. For the smaller $\mathrm{Cu}-\mathrm{TF}$ ST-FNSF devices, more of the divertor coils must be normally conducting due to nuclear heating and damage issues.

Building on the TF and PF coil layouts found to be optimal for the $\mathrm{Cu}$-TF ST-FNSF configuration, net-electricity producing pilot plants utilizing HTS TF magnets have been systematically studied as a function of aspect ratio and inboard shielding thickness. To achieve peak outboard neutron fluence $>6 \mathrm{MWy} \mathrm{m} \mathrm{m}^{-2}$ for the FNSF mission, approximately $60 \mathrm{~cm}$ of inboard WC-equivalent shield is needed to reduce radiation damage to the HTS TF magnets to acceptable levels. For shields in this thickness range, $R_{0}=3 \mathrm{~m}$ is a favorable plasma major radius size for achieving $Q_{\text {eng }}>1$ for a wide range of aspect ratios and shielding thicknesses. Lower aspect ratios with $A=1.6$ to 2 are found to maximize the fusion power per unit $\mathrm{TF}$ (and $\mathrm{OH}$ ) coil volume for all shielding thicknesses, while higher $A=2.5-3$ would minimize blanket volume for otherwise similar overall fusion performance. As for the $\mathrm{Cu}$-TF ST-FNSF, long-leg/super-X divertor scenarios in low-A HTS-TF FNSF/pilot plants are found to substantially reduce peak divertor heat fluxes. For low-A devices to be attractive at the $R_{0}=3 \mathrm{~m}$ scale, high HTS winding pack current densities $\geqslant 40-70 \mathrm{MA} \mathrm{m}^{-2}$ and peak fields up to $18 \mathrm{~T}$ are needed to provide space for shielding, but if such magnets could be fabricated, an $R_{0}=3 \mathrm{~m}, A=2$ all superconducting FNSF/pilot plant with poloidally continuous TF coils (no joints), all equilibrium PF coils outside the TF, and a small solenoid for current initiation would be feasible and could support fusion powers at the 500-600 MW level. Such a device would be capable of $6 \mathrm{MWy} \mathrm{m}^{-2}$ (peak), $Q_{\mathrm{eng}}>1, \mathrm{TBR} \approx 1$ (assuming a thin inboard breeding blanket), and would have significantly reduced TF magnet volume (relative to conventional aspect ratio) which could help reduce overall magnet cost.

\section{Acknowledgments}

This work was supported primarily by the U.S. DOE Contract Number DE-AC02-09CH11466. CCFE authors were funded by the Research Councils UK Energy Programme grant number EP/I501045. Configuration development for a thinshield $R_{0}=1.4 \mathrm{~m}$ HTS ST pilot plant concept [170] was supported by Tokamak Energy, LTD (UK). The digital data for this paper can be found in: http://arks.princeton.edu/ ark:/88435/dsp01pn89d906g

\section{References}

[1] Neilson G.H. et al 2013 Fusion Sci. Technol. 64463

[2] Waganer L., Najmabadi F. and Tillack M. 1995 What must DEMO do? IEEE Proc. of 16th Int. Symp. on Fusion Engineering vol 2 (IEEE) pp 1157-61

[3] Menard J. et al 2011 Nucl. Fusion 51103014

[4] Peng Y.-K.M. et al 2005 Plasma Phys. Control. Fusion 47 B263

[5] Peng Y.-K.M. et al 2009 Fusion Sci. Technol. 56957

[6] Voss G. et al 2008 Fusion Eng. Des. 831648

[7] Kotschenreuther M., Valanju P., Mahajan S. and Schneider E. 2009 Fusion Eng. Des. 8483

[8] Kuteev B. et al 2011 Nucl. Fusion 51073013

[9] Chan V., Stambaugh R. and Garofalo A. 2010 Fusion Sci. Technol. $\mathbf{5 7} 66$

[10] Garofalo A. et al 2014 Nucl. Fusion 54073015

[11] Abdou M. et al 1996 Fusion Technol. 291

[12] Fishpool G. et al 2013 J. Nucl. Mater. 438 S356

[13] Goldston R., White R. and Boozer A. 1981 Phys. Rev. Lett. 47647

[14] Pankin A. et al 2004 Comput. Phys. Commun. 164421

[15] Pankin A., Mccune D., Andre R., Bateman G. and Kritz A. 2004 Comput. Phys. Commun. 159157

[16] Huba J.D. 2013 NRL Plasma Formulary (Washington, DC: Naval Research Laboratory)

[17] Menard J., Jardin S., Kaye S., Kessel C. and Manickam J. 1997 Nucl. Fusion 37595

[18] Wong C., Wesley J., Stambaugh R. and Cheng E. 2002 Nucl. Fusion 42547

[19] Menard J.E. et al 2003 Unified ideal stability limits for advanced tokamak and spherical torus plasmas Technical Report PPPL-3779

[20] Menard J.E. et al 2004 Phys. Plasmas 11639 
[21] Najmabadi F. 2003 Fusion Eng. Des. 65143

[22] Peng Y. et al 2011 Fusion nuclear science facility (FNSF) 2011 IEEE/NPSS 24th Symp. on Fusion Engineering (IEEE) pp 1-7

[23] Lumsdaine A. and Peng M. 2011 Structural analysis of an optimally designed spherical tokamak centerpost 2011 IEEE/NPSS 24th Symp. on Fusion Engineering (IEEE) pp $1-6$

[24] Lumsdaine A., Tipton J. and Peng M. 2012 Fusion Eng. Des. 871190

[25] Roberto M. and Galvao R. 1992 Nucl. Fusion 321666

[26] Kolemen E. et al 2011 Nucl. Fusion 51113024

[27] Menard J. et al 2012 Nucl. Fusion 52083015

[28] Gerhardt S., Andre R. and Menard J. 2012 Nucl. Fusion 52083020

[29] Crowell J.A. and Blanchard J.P. 2000 Electromagnetic disruption effects in the ARIES-RS tokamak design Technical Report University of Wisconsin-Madison, Report Number UWFDM-1148

[30] Jordan T. 1998 Fusion Eng. Des. 43173

[31] Jordan T. and Schneider D. 1996 Fusion Eng. Des. 31313

[32] Menard J. http://nstx-u.pppl.gov/software/lrdfit

[33] Berzak Hopkins L. et al 2012 Nucl. Fusion 52063025

[34] Menard J. et al 2006 Phys. Rev. Lett. 97095002

[35] Hofmann F., Moret J.-M. and Ward D. 1998 Nucl. Fusion 381767

[36] Ward D. and Hofmann F. 1994 Nucl. Fusion 34401

[37] Humphreys D. et al 2009 Nucl. Fusion 49115003

[38] Inho S. et al 2014 IEEE Trans. Appl. Supercond. 241

[39] Kessel C., Mau T., Jardin S. and Najmabadi F. 2006 Fusion Eng. Des. 8063

[40] Elguebaly L. 2006 Fusion Eng. Des. 8099

[41] Mergia K. and Boukos N. 2008 J. Nucl. Mater. 3731

[42] Malang S., Tillack M., Wong C.P.C., Morley N. and Smolentsev S. 2011 Fusion Sci. Technol. 60249

[43] Gerhardt S. et al 2013 Nucl. Fusion 53043020

[44] Sabbagh S.A. et al 2002 Phys. Plasmas 92085

[45] Paoletti F. et al 2002 Nucl. Fusion 42418

[46] Sabbagh S. et al 2006 Nucl. Fusion 46635

[47] Menard J. et al 2014 Phys. Rev. Lett. 113255002

[48] Berkery J. et al 2015 Nucl. Fusion 55123007

[49] Bondeson A. and Ward D. 1994 Phys. Rev. Lett. 722709

[50] Betti R. and Freidberg J. 1995 Phys. Rev. Lett. 742949

[51] Boozer A.H. 1995 Phys. Plasmas 24521

[52] Sontag A.C. et al 2005 Phys. Plasmas 12056112

[53] Sontag A.C. et al 2007 Nucl. Fusion 471005

[54] Liu Y., Chu M.S., Gimblett C.G. and Hastie R.J. 2008 Phys. Plasmas 15092505

[55] Liu Y., Chu M.S., Chapman I.T. and Hender T.C. 2008 Phys. Plasmas 15112503

[56] Berkery J.W. et al 2010 Phys. Rev. Lett. 104035003

[57] Berkery J.W. et al 2010 Phys. Plasmas 17082504

[58] Menard J. et al 2010 Nucl. Fusion 50045008

[59] Berkery J. et al 2011 Phys. Rev. Lett. 1063

[60] Bialek J., Boozer A.H., Mauel M.E. and Navratil G.A. 2001 Phys. Plasmas 82170

[61] Sabbagh S. et al 2004 Nucl. Fusion 44560

[62] Sabbagh S. et al 2006 Phys. Rev. Lett. 97045004

[63] Sabbagh S. et al 2010 Nucl. Fusion 50025020

[64] Katsuro-Hopkins O., Bialek J., Maurer D. and Navratil G. 2007 Nucl. Fusion 471157

[65] Katsuro-Hopkins O., Sabbagh S. and Bialek J. 2009 Analysis of resistive wall mode LQG control in NSTX with mode rotation Proc. of the 48h IEEE Conf. on Decision and Control (CDC) held jointly with 2009 28th Chinese Control Conf. (IEEE) pp 309-14

[66] ITER Physics Expert Group on Confinement, Transport, Confinement Modelling, Database and ITER Physics Basis Editors 1999 Nucl. Fusion 392175
[67] Doyle E. et al 2007 Nucl. Fusion 47 S18

[68] Kaye S. et al 2007 Nucl. Fusion 47499

[69] Kaye S. et al 2007 Phys. Rev. Lett. 981

[70] Valovic M. et al 2009 Nucl. Fusion 49075016

[71] Valovic M. et al 2011 Nucl. Fusion 51073045

[72] Luce T.C., Petty C.C. and Cordey J.G. 2008 Plasma Phys. Control. Fusion $\mathbf{5 0} 043001$

[73] Petty C.C. 2008 Phys. Plasmas 15080501

[74] Greenwald M. et al 1988 Nucl. Fusion 282199

[75] Greenwald M. 2002 Plasma Phys. Control. Fusion 44 R27

[76] Maingi R. et al 2009 J. Nucl. Mater. 390-1 440

[77] Maingi R. et al 2010 Phys. Rev. Lett. 105135004

[78] Gerhardt S. et al 2014 Nucl. Fusion 54083021

[79] Ono M. 1995 Phys. Plasmas 24075

[80] Menard J., Majeski R., Kaita R., Ono M. and Munsat T. 1999 Phys. Plasmas 62002

[81] Taylor G. et al 2010 Phys. Plasmas 17056114

[82] Hosea J. et al 2008 Phys. Plasmas 15056104

[83] Phillips C. et al 2009 Nucl. Fusion 49075015

[84] Green D. et al 2011 Phys. Rev. Lett. 1071

[85] Perkins R. et al 2012 Phys. Rev. Lett. 1091

[86] Perkins R. et al 2013 Nucl. Fusion 53083025

[87] Bertelli N. et al 2014 Nucl. Fusion 54083004

[88] Rosenberg A.L. et al 2004 Phys. Plasmas 112441

[89] Liu D. et al 2010 Plasma Phys. Control. Fusion 52025006

[90] Harvey R.W. et al 2014 AIP Conf. Proc. 1580314

[91] Taylor G. et al 2012 Phys. Plasmas 19042501

[92] Taylor G. et al 2004 Phys. Plasmas 114733

[93] Preinhaelter J. 2005 AIP Conf. Proc. 787349

[94] Urban J. et al 2011 Nucl. Fusion 51083050

[95] Jones B. et al 2003 Phys. Rev. Lett. 901

[96] Diem S. et al 2009 Nucl. Fusion 49095027

[97] Diem S. et al 2009 Phys. Rev. Lett. 1031

[98] Kotschenreuther M. et al 2000 Nucl. Fusion 40677

[99] Kaye S. et al 2009 Nucl. Fusion 49045010

[100] Yuh H.Y. et al 2009 Phys. Plasmas 16056120

[101] Janev R., Boley C. and Post D. 1989 Nucl. Fusion 292125

[102] Hemsworth R. 2003 Nucl. Fusion 43851

[103] Von Halle A. et al 1993 TPX neutral beam injection system design 15th IEEE/NPSS Symp. Fusion Engineering vol 1 (IEEE) pp 212-5

[104] Pamela J. 1995 Plasma Phys. Control. Fusion 37 A325

[105] Bacal M. 2006 Nucl. Fusion 46 S250

[106] Kojima A. et al 2010 Rev. Sci. Instrum. 81 $02 B 112$

[107] Kojima A. et al 2011 Nucl. Fusion 51083049

[108] Kojima A. et al 2014 Rev. Sci. Instrum. 8502 B312

[109] Hemsworth R. et al 2009 Nucl. Fusion 49045006

[110] Franzen P. et al 2013 Fusion Eng. Des. 883132

[111] Ivanov A.A. et al 2014 Rev. Sci. Instrum. 85 02 B 102

[112] Start D.F.H., Cordey J.G. and Jones E.M. 1980 Plasma Phys. 22303

[113] Cordey J.G. 1984 Plasma Phys. Control. Fusion 26123

[114] Fisch N. 1987 Rev. Mod. Phys. 59175

[115] Gerhardt S. et al 2011 Nucl. Fusion 51033004

[116] Strachan J. et al 1993 Nucl. Fusion 33991

[117] Bickerton R.J., Connor J.W. and Taylor J.B. 1971 Nat. Phys. Sci. 229110

[118] Zarnstorff M. et al 1988 Phys. Rev. Lett. 601306

[119] Wilson H. 1992 Nucl. Fusion 32257

[120] Podesta M. et al 2013 Phys. Plasmas 20082502

[121] Fredrickson E. et al 2014 Nucl. Fusion 54093007

[122] Najmabadi F. et al 2006 Fusion Eng. Des. 803

[123] Canik J.M., Gray T.K., Maingi R. and Menard J.E. 2013 Power and particle exhaust in an ST-FNSF IEEE 25th Symp. on Fusion Engineering (IEEE) pp 1-6

[124] Ryutov D.D. 2007 Phys. Plasmas 14064502

[125] Kotschenreuther M., Valanju P.M., Mahajan S.M. and Wiley J.C. 2007 Phys. Plasmas 14072502 
[126] Tenney F. 1976 A tokamak hybrid study Technical Report Princeton Plasma Physics Laboratory, Report Number 1284

[127] Valanju P.M., Kotschenreuther M., Mahajan S.M. and Canik J. 2009 Phys. Plasmas 16056110

[128] Kotschenreuther M. et al 2010 Nucl. Fusion 50035003

[129] Herrmann A. 2002 Plasma Phys. Control. Fusion 44883

[130] Labombard B. 1997 J. Nucl. Mater. 241-3 149

[131] Loarte A. 2001 Plasma Phys. Control. Fusion 43 R183

[132] Soukhanovskii V.A. et al 2012 Phys. Plasmas 19082504

[133] Ahn J.-W. and Counsell G. 2001 J. Nucl. Mater. 290-3 820

[134] Counsell G.F. et al 2002 Plasma Phys. Control. Fusion 44 B23

[135] Paul S., Maingi R., Soukhanovskii V., Kaye S. and Kugel H. 2005 J. Nucl. Mater. 337-9 251

[136] Turnbull A.D., Lin-Liu Y.R., Miller R.L., Taylor T.S. and Todd T.N. 1999 Phys. Plasmas 61113

[137] Tanaka K. et al 1994 IEEE Trans. Magn. 302511

[138] Tanaka K. et al 2004 IEEE Trans. Appl. Supercond. 14402

[139] Bird M.D. 2004 Supercond. Sci. Technol. 17 R19

[140] Weggel R., Pearson C. and King B. 2001 Design study for 20 $\mathrm{T} 15 \mathrm{~cm}$ bore hybrid magnet with radiation-resistant insert for pion capture Proc. of the 2001 Particle Accelerator Conf. vol 5 (IEEE) pp 3398-400 (Cat. No.01CH37268)

[141] Muzzi L., De Marzi G., Di Zenobio A. and Della Corte A. 2015 Supercond. Sci. Technol. 28053001

[142] Ilyin Y. et al 2010 IEEE Trans. Appl. Supercond. 20415

[143] Lim B. et al 2011 IEEE Trans. Appl. Supercond. 211918

[144] Everitt D. et al 2013 ITER Central Solenoid design IEEE 25th Symp. on Fusion Engineering (IEEE) pp 1-8

[145] Kim K. et al 2015 Fusion Eng. Des. 96-7 281

[146] Takayasu M., Chiesa L., Bromberg L. and Minervini J.V. 2012 Supercond. Sci. Technol. 25014011

[147] van der Laan D.C., Noyes P.D., Miller G.E., Weijers H.W. and Willering G.P. 2013 Supercond. Sci. Technol. 26045005

[148] Eich T. et al 2011 Phys. Rev. Lett. 107215001

[149] Goldston R. 2012 Nucl. Fusion 52013009

[150] Makowski M.A. et al 2012 Phys. Plasmas 19056122

[151] Eich T. et al 2013 Nucl. Fusion 53093031

[152] Meier E., Gerhardt S., Menard J., Rognlien T. and Soukhanovskii V. 2015 Nucl. Fusion 55086002

[153] Schneider R. et al 2006 Contrib. Plasma Phys. 463

[154] Braams B.J. 1996 Contrib. Plasma Phys. 36276

[155] Reiter D., Baelmans M. and Börner P. 2005 Fusion Sci. Technol. 47172

[156] Martin Y.R., Takizuka T. and Group T.I.C.H.-M.T.D. 2008 J. Phys.: Conf. Ser. 123012033

[157] Labombard B. et al 2015 Nucl. Fusion 55053020

[158] Hawryluk R. 1981 An empirical approach to tokamak transport Physics Plasmas Close to Thermonuclear Conditions: Int. School of Plasma Physics (Varenna, Italy, 27 August-8 September 1979) vol 1, ed B. Coppi et al (Oxford: Commission of the European Communities) pp 19-46

[159] Ongena J., Evrard M. and Mccune D. 2004 Fusion Sci. Technol. 45371

[160] Mueller D. 2013 Phys. Plasmas 20058101

[161] Raman R. and Shevchenko V.F. 2014 Plasma Phys. Control. Fusion 56103001

[162] Raman R. et al 2014 IEEE Trans. Plasma Sci. 422154

[163] Raman R. 2015 Fusion Sci. Technol. 68 674-9

[164] Raman R. et al 2001 Nucl. Fusion 411081

[165] Schaffer M., Maingi R., Hyatt A. and Mahdavi M.A. 1996 Nucl. Fusion $\mathbf{3 6} 495$

[166] Clinard F., Hurley G. and Hobbs L. 1982 J. Nucl. Mater. 108-9 655
[167] Schultz J. 1981 Sizing of the thermal and electrical systems for an FED bundle divertor design with $\mathrm{MgO}$ insulation Technical Report DOE/ET/51013-2

[168] Brown T. et al 2013 Progress in developing the STFNSF configuration IEEE 25th Symp. on Fusion Engineering (IEEE) pp 1-6

[169] El-Guebaly L. et al 2014 IEEE Trans. Plasma Sci. 421457

[170] Brown T., Menard J., El-Gueblay L. and Davis A. 2015 Fusion Sci. Technol. 68277

[171] Snyder P. et al 2004 Nucl. Fusion 44320

[172] Snyder P.B., Wilson H.R., Osborne T.H. and Leonard A.W. 2004 Plasma Phys. Control. Fusion 46 A131

[173] Diallo A. et al 2011 Nucl. Fusion 51103031

[174] Diallo A. et al 2013 Nucl. Fusion 53093026

[175] Ono M. et al 2015 Nucl. Fusion 55073007

[176] Yushmanov P. 1983 Nucl. Fusion 231599

[177] Mcclements K.G. and Hole M.J. 2012 Phys. Plasmas 19072514

[178] Urano H. et al 2006 Plasma Phys. Control. Fusion 48 A193

[179] Shinohara K. et al 2007 Nucl. Fusion 47997

[180] Saibene G. et al 2007 Nucl. Fusion 47969

[181] Los Alamos National Laboratory 2015 A general Monte Carlo N-particle (MCNP) transport code https://mcnp.lanl.gov/

[182] University of Wisconsin-Madison Fusion Technology Institute 2015 DAGMC users guide http://svalinn.github. io/DAGMC/usersguide/

[183] Simon N. 1995 Radiation limits for $\mathrm{Nb}_{3} \mathrm{Sn}$ superconductors for ITER magnets: a literature review Technical Report Oak Ridge Operations, Oak Ridge, TN

[184] Kuroda K. et al 1995 J. At. Energy Soc. Japan 37652

[185] Nishimura A. et al 2009 Fusion Eng. Des. 841425

[186] Schultz J. 2003 Radiation resistance of fusion magnet materials 20th IEEE/NPSS Symp. on Fusion Engineering (IEEE) pp 423-6

[187] Nishimura A., Izumi Y., Imaizumi M. and Nishijima S. 2012 AIP Conf. Proc. 1435 99-106

[188] 2009 ITER Magnet Superconducting and Electrical Design Criteria (ITER D 22GRQH v12)

[189] Boutboul T. et al 2016 IEEE Trans. Appl. Supercond. 261

[190] Baumgartner T. et al 2015 Sci. Rep. 510236

[191] Zinkle S.J. 2005 Phys. Plasmas 12058101

[192] Zinkle S.J. and Busby J.T. 2009 Mater. Today 1212

[193] Zinkle S., Möslang A., Muroga T. and Tanigawa H. 2013 Nucl. Fusion $\mathbf{5 3} 104024$

[194] Stork D. et al 2014 Fusion Eng. Des. 891586

[195] Fabritsiev S., Zinkle S. and Singh B. 1996 J. Nucl. Mater. 233-7 127

[196] Reiersen W., Dahlgren F., Fan H., Neumeyer C. and Zatz I. 2003 Fusion Eng. Des. 65303

[197] Tobita K. et al 2007 Nucl. Fusion 47892

[198] Ni M., Wang Y., Yuan B., Jiang J. and Wu Y. 2013 Fusion Eng. Des. 882422

[199] Baker N., Mckee B. and Mcnab I. 1986 IEEE Trans. Magn. 221386

[200] Werst M., Brunson G., Hsieh K., Sledge R. and Weldon W. 1989 Design of a prototype 20 Tesla, single turn, toroidal field coil for the fusion ignition experiment (IGNITEX) IEEE 13th Symp. on Fusion Engineering (IEEE) pp 43-6

[201] Hsieh K., Driga M., Weldon W. and Werst M. 1989 Electromechanical analysis of a prototype $20 \mathrm{~T}$, single turn toroidal field coil for IGNITEX IEEE 13th Symp. on Fusion Engineering (IEEE) pp 1138-41

[202] Werst M., Ingram S., Wehrlen D. and Weldon W. 1994 IEEE Trans. Magn. 302550

[203] Hong B. et al 2011 Nucl. Fusion 51113013

[204] Gi K. et al 2015 Nucl. Fusion 55063036

[205] Windsor C., Morgan J. and Buxton P. 2015 Nucl. Fusion 55023014

[206] Sorbom B. et al 2015 Fusion Eng. Des. 100378 
[207] Gates D. et al 2007 Nucl. Fusion 471376

[208] Lazarus E.A. et al 1991 Phys. Fluids B: Plasma Phys. 32220

[209] Zohm H. et al 2013 Nucl. Fusion 53073019

[210] Schwartz J. 1992 J. Fusion Energy 1119

[211] Barth C., Mondonico G. and Senatore C. 2015 Supercond. Sci. Technol. 28045011

[212] Prokopec R., Fischer D.X., Weber H.W. and Eisterer M. 2015 Supercond. Sci. Technol. 28014005
[213] El-Guebaly L.A. 2003 Fusion Eng. Des. 65263

[214] Costley A., Hugill J. and Buxton P. 2015 Nucl. Fusion 55033001

[215] Gryaznevich M., Shevchenko V. and Sykes A. 2006 Nucl. Fusion 46 S573

[216] Tobita K. et al 2006 Fusion Eng. Des. 811151

[217] Nishitani T., Yamauchi M., Nishio S. and Wada M. 2006 Fusion Eng. Des. 811245 